\title{
Sustainable sorbitol-derived compounds for gelation of the full range of ethanol-water mixtures
}

\author{
Glenieliz C. Dizon, ${ }^{a, b}$ George Atkinson, ${ }^{a, b}$ Stephen P. Argent, ${ }^{a}$ Lea T. Santu a,b and David B. \\ Amabilino*a,b \\ a School of Chemistry, University of Nottingham, University Park, NG7 2RD, UK. E- \\ mail: david.amabilino@nottingham.ac.uk \\ ${ }^{\circ}$ The GSK Carbon Neutral Laboratories for Sustainable Chemistry, University of Nottingham, Triumph Road, NG7 \\ 2TU, UK
}

\begin{abstract}
During the development of soft material systems inspired by green chemistry, we show that naturally occurring starting materials can be used to prepare mono- and dibenzylidene sorbitol derivatives. These compounds gelate a range of organic, aqueous (including with mono and divalent metal salt solutions) and ethanolic (ethanol-water) solutions, with the equimolar mixture of two of the gelators gelling all compositions from $100 \%$ ethanol to $100 \%$ water (something neither of the individual components do). We explored the influence of modifications to the acetal substituents on the formation of the compounds as well as the impact of steric bulk on self-assembly properties of the gelators. The effect of solvent on the self-assembly, morphology, and rheology of the 1,3:2,4-di(4isopropylbenzylidene)-D-sorbitol (DBS-iPr), 2,4(4-isopropylbenzylidene)-D-sorbitol (MBS$\mathrm{iPr}$ ) and the equimolar multicomponent (DBS-MBS-iPr) gels have been investigated. DBSiPr gelates polar solvents to form smooth flat fibres, whereas in non-polar solvents such as cyclohexane helical fibres grow where the chirality is determined by the stereochemistry of the sugar. Oscillatory rheology revelaed that MBS-iPr gels have appreciable strength and elasticity, in comparison to DBS-iPr gels, regardless of the solvent medium employed. Powder X-ray diffraction was used to probe the arrangement of the gelators in the xerogels they form, and two single crystal X-ray structures of related MBS derivatives give the first precise structural information concerning layering and hydrogen bonding in the monobenzylidene compounds. This kind of layering could explain the apparent self-sorting behaviour of the DBS-MBS-iPr multicomponent gels. The combination of sorbitol-derived gelators reported in this work could find potential applications as multicomponent systems, for example, in soft materials for personal care products, polymer nucleation/clarification, and energy technology.
\end{abstract}




\section{Introduction}

As we face challenging environmental problems, interest in sustainable solutions in all areas of science is growing, and in the context of this work in particular in the field of solgel science. ${ }^{1}$ Within this area, a contemporary challenge is to target existing sustainable feedstocks for the synthesis of supramolecular gelators. ${ }^{2,3}$ The incorporation of environmentally friendly approaches guided by the principles of green chemistry can give alternatives to the more conventional chemical designs, reducing or eliminating the use of hazardous and toxic solvents. ${ }^{4,5} \mathrm{~A}$ wide range of naturally occurring molecular motifs including polysaccharides (alginates ${ }^{6}$ and carrageenans $^{7}$ ), ureas, $^{8}$ sugars, $^{9-11}$ steroids, $^{12}$ peptides $^{13}$, acids (for example gallic acid derivatives ${ }^{14-16}$ ) and other naturally occurring compounds have proven their potential as gelators. ${ }^{17}$

Low molecular weight gelators (LMWGs) have gained increasing attention as an alternative to polymer derived gels, because LMWGs can be more responsive to stimuli which therefore aid in modifying, enhancing or developing additional desired properties to the systems. The syntheses of derivatives of these naturally occurring compounds and identifying LMWGs has been the focal point in the field of sustainable gelators. ${ }^{18,19}$

Sugar-based gelators, ${ }^{20}$ such as sorbitol derivatives, especially those based on dibenzylidene-D-sorbitol (DBS), are of much interest for their facile preparation and many applications. ${ }^{21}$ DBS derivatives are the product of a condensation reaction of D-sorbitol and a benzaldehyde in the presence of an acid catalyst. The DBS molecules are believed to self-assemble by adopting a 'butterfly' conformation, with two aromatic 'wings' on either side of an aliphatic body and being held together by hydrogen bonds, ${ }^{22}$ although no direct structural evidence exists to date.

The exact nature of the self-assembly of DBS derivatives upon gelation has been the subject of much research and debate. ${ }^{23}$ It has been suggested that DBS compounds undergo self-assembly in appropriate solvents through relatively weak intermolecular interactions such as hydrogen bonding, electrostatic, van der Waals, $\Pi-\Pi$ stacking or solvophobic interactions if in a polar solvent, all have been inferred. ${ }^{23}$ It is clear from the morphology of the xerogels that the molecules self-assemble into fibrous networks like other LMWGs. ${ }^{24-26}$ DBS is an important LMWG, given the molecule's versatility in gelling a range of organic solvents ${ }^{27}$ and polymers ${ }^{28}$ (where it creates a dual network of covalent and non-covalent systems) a range of concentrations and temperatures. ${ }^{29,30}$

The scope of this paper is to explore the synthesis and characteristics of sorbitol gelators and the gels they form. Three sustainable aromatic aldehydes have been employed in the condensation reaction with sorbitol and the effect of molecular structure on the properties of the resulting gelators was investigated. The microstructure of the xerogels that result from evaporation of a range of solvents was studied by scanning electron microscopy (SEM) and their structural arrangement by powder X-ray diffraction (PXRD) and Fourier transform infrared spectroscopy (FTIR). This work is beneficial for designing gelators with sustainable components, because of their potential application in the food or personal care sector given that the compounds that are decomposed by acid to the edible components, are relatively benign materials, already in used in cosmetic applications. ${ }^{31,32}$ 


\section{Results and Discussion}

The benzylidene sorbitol derivatives were synthesised from D-sorbitol and sustainable aldehydes (cuminaldehyde, cinnamaldehyde, and vanillin) in the presence of catalytic 4toluenesulfonic acid to yield white crystalline materials (

Scheme 1). This equilibrium condensation reaction can yield monobenzylidene sorbitol (MBS), dibenzylidene sorbitol (DBS) and tribenzylidene sorbitol (TBS) compounds depending on a range of factors such as the stoichiometry, $\mathrm{pH}$, aldehyde and solubility of the starting materials and the intermediates. ${ }^{23,33}$ All reactions were done using 2.0 equivalents of the aromatic aldehyde with respect to sorbitol, except for the preparation of MBS-iPr which was carried out using 1.0 equivalent of cuminaldehyde.

The sorbitol derivatives in this research were isolated in yields of $45-75 \%$ (with respect to sorbitol being the limiting reagent) from reactions performed at room temperature. The di- substituted sorbitol product is clearly disfavoured in all cases, only the compound derived from cuminaldehyde gave isolable yields of the DBS in our hands. Contrarily, all the MBS products could be isolated in yields superior to $58 \%$. Reactions involving vanillin required an inert atmosphere because of oxidation of the compound noted by colouration of the reaction performed in air. Products were isolated via filtration and characterised by NMR, HRMS, optical rotation and FTIR spectroscopy, and were confirmed to be chemically pure by elemental analysis (see ESI for details). Circular dichroism was also used to show the molecular chirality (see ESI), although the technique could not be used to study aggregation because of light scattering from the samples. A fifth aldehyde was investigated for condensation with the D-sorbitol, vanillin acetate. This aldehyde was reacted with sorbitol and 4-toluenesulfonic acid under both the room temperature conditions recommended by Gardlik ${ }^{34}$ and the Dean-Stark procedure. ${ }^{35,36}$ In both cases, the acetal group of the vanillin acetate deprotected under the acidic conditions, and the final product was MBS-Van (58\%).

Furthermore, an attempt was made to synthesise hetero-acetal gelator by reacting a mono-acetal with an aldehyde. MBS-Van was chosen as the mono-acetal and cuminaldehyde as the other reagent, the latter was chosen because it was the only aldehyde screened which had formed the di-acetal. Milder reaction conditions were chosen (Gardlik's ${ }^{34}$ method at room temperature) in an attempt to minimise the reverse reaction of the mono-acetal back to vanillin and sorbitol. Analysis of the reaction mixture by timeof-flight mass spectrometry showed the substitution of aldehydes was occurring (ESI Fig 14). We believe that even these mildly acidic conditions cause equilibration of the monoacetal that undergoes the reverse reaction to vanillin and sorbitol, which then goes on to react with the cuminaldehyde and forms MBS-iPr. This hypothesis is supported by the reaction mixture turning purple after being exposed to air for a short time, which is indicative of the presence of unreacted vanillin (MBS-Van does not oxidise readily in air in our experience). 

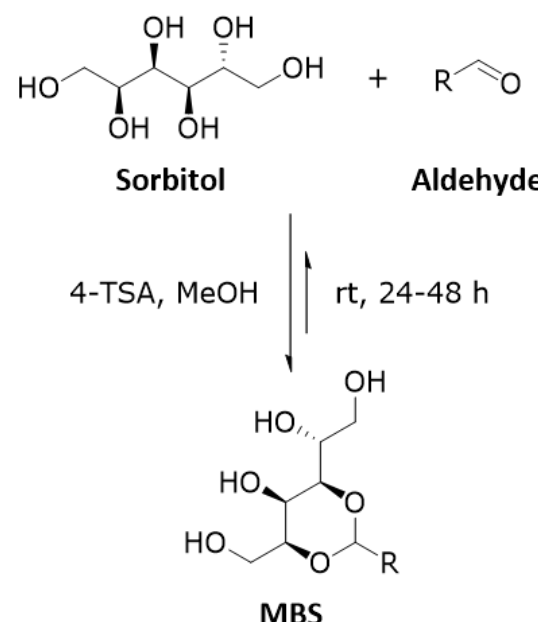<smiles>CC1CCCCC1</smiles><smiles>[R]C1OC2COC([R])OC2C(C(O)CO)O1</smiles>

DBS

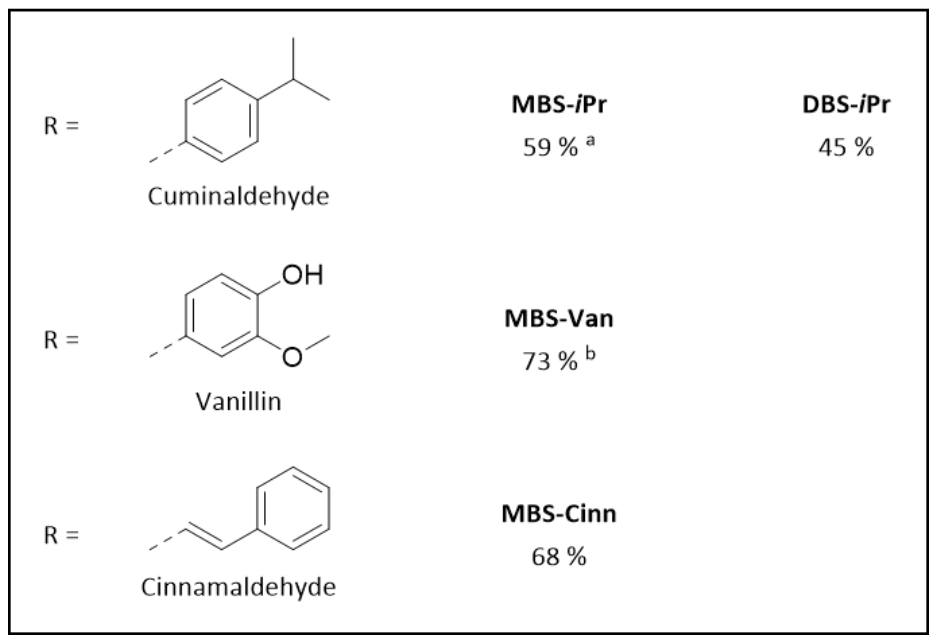

Scheme 1| The synthesis of DBS-iPr, MBS-Cinn, MBS-Van and MBS-iPr. Reactions were done in air using 2.0 eq. of the aromatic aldehyde except; a) reaction carried out using 1.0 eq. of the aromatic aldehyde for $12 \mathrm{~h}$; b) reaction performed under inert atmosphere.

The preparation of DBS-iPr was reported in a patent ${ }^{36}$ where the inventors used $\mathrm{C}_{9}$ alkylbenzenesulfonic acid, dimethylsulfoxide, benzene and iso-propanol as the reaction medium, apparently obtaining a high yield of the product. There is no specific procedure for the synthesis of MBS-iPr or its characterisation in the literature, although, an asymmetric syntheses of diacetal compounds where MBS-iPr could be a reagent is contained in a patent. ${ }^{37}$ The reactions that produced MBS-Cinn and MBS-Van did not yield isolable amounts of the desired diacetal. The selectivity of these reactions to result in the mono-acetal is quite remarkable in our view. Experiments have been run over weeks, at elevated temperatures $\left(60-100^{\circ} \mathrm{C}\right)$, under inert atmosphere and with excess of aldehyde 
in an attempt to force production of the di-acetals, but all of these resulted only in monoacetal formation. Mass spectra of the reaction mixtures inevitably show a strong monoacetal peak and a very small di-acetal peak (ESI Fig 14). Clearly, the formation of the diacetals derived from these two compounds is unfavourable under the reaction conditions employed. As for MBS-iPr, we could not locate a synthetic procedure or characterisation for MBS-Van, although its antioxidant activity against free radicals and anti-inflammatory property were patented recently. ${ }^{38}$

The condensation of the aldehyde with sorbitol is proposed to proceed via the solvent acetals. ${ }^{39}$ Song et al. ${ }^{35}$ noted that aromatic aldehydes with electron-donating substituents were harder to react with sorbitol than ones with electron-withdrawing substituents, although they do not provide a rationale. Kobayashi's ${ }^{40}$ work suggests that the reaction mechanism for the formation of the di-acetal proceeds via nucleophilic attack of the carbocation on the protonated aldehyde (

Fig. 1). ${ }^{41}$ From this proposed mechanism, it appears that electron-donating substituents on the aromatic ring would reduce the electrophilicity of the carbon atom attached to the phenyl ring.

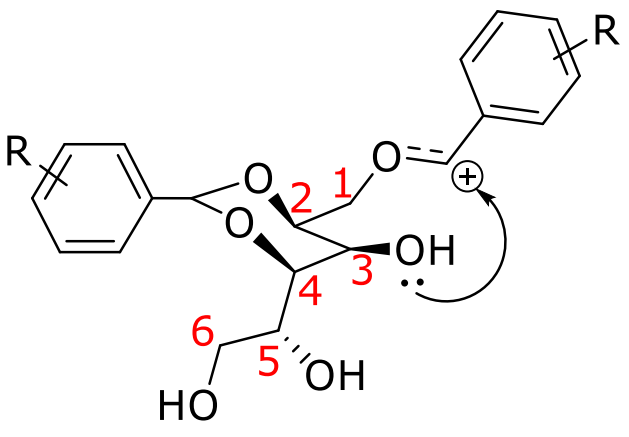

Fig. 1 | The proposed final step in DBS formation ${ }^{41}$

This inductive effect will slow the rate of the forward reaction. Possibly, the aldehyde will detach from the sorbitol before the nucleophilic attack of a second aldehyde can occur because the system is under equilibrium conditions. However, this hypothesis does not account for the observation of the relatively rapid precipitation of a white solid in the reaction vessels, indicating that the formation of the mono-acetal is not particularly inhibited - which it surely would be if the above hypothesis was correct, as it forms through the same mechanism. It is also possible that the insoluble nature of the intermediate halts the reaction and freezes the equilibrium. The exact origin of the effect will require further investigation beyond the scope of the present work, but an important conclusion of this research is that MBS and DBS derivatives of electron deficient aldehydes can be isolated independently.

\section{Crystallography}

Single crystals of both MBS-Van and MBS-Cinn were obtained via crystalisation from aqueous solutions of $\mathrm{KCl}$ and $\mathrm{CaCl}_{2}(2 \% \mathrm{w} / \mathrm{v})$; heating and cooling over a period of 48 hours to afford needle-like crystals. To date, there have been no single crystal structures reported in the literature for either MBS or DBS and their derivatives (to 
the best of our knowledge) which makes the following observations useful for the field, although we should emphasise that these derivatives that crystallise do not apparently form gels. The packing of MBS-Van (Fig. 2 and ESI Fig. 38, ) and MBSCinn (Fig. 3 and ESI Fig. 38, ) are similar overall, where sugar and aromatic layers alternate. The asymmetric unit of MBS-Van contains one molecule whereas the asymmetric unit of MBS-Cinn contains two distinct molecules possessing the same type of conformation. Location and refinement of the hydroxyl hydrogen atoms were handled differently in the two structures (full details are in the ESI and relevant sections of the CIFs).

MBS-Van crystallises in the space group $P 2_{1}$ (monoclinic crystal system) with neighbouring molecules in $Y$-packing motif (ESI Fig. 38). ${ }^{42}$ Crystals of MBS-Van diffracted strongly; three of the four hydroxyl hydrogen atoms were located in the electron density map and their positions were refined. Intermolecular hydrogen bonds (Fig. 2, summarised in ESI Table 1) are observed between adjacent sugar hydroxyl groups with $\mathrm{H} \cdots \mathrm{A}$ separations of $1.84,1.87$, and $1.93 \AA$ for pairs $\mathrm{O} 4-\mathrm{H} 4 \cdots \mathrm{O} 8$, $\mathrm{O} 8-\mathrm{H} 8 \cdots \mathrm{O} 4$ and $\mathrm{O} 12-\mathrm{H} 12 \cdots \mathrm{O} 2$, respectively. The $[\mathrm{O} \cdots \mathrm{H} \cdots \mathrm{O}]$ angles between the molecules are $165.1^{\circ}, 155.9^{\circ}$ and $174.9^{\circ}$, respectively. The final hydroxyl hydrogen atom $(\mathrm{O} 2-\mathrm{H} 2)$, was geometrically placed to donate a hydrogen bond to the closest suitable acceptor; detailed discussion of the hydrogen bond geometry is not warranted. Furthermore, there are two additional less evident interactions present on the vanillin aromatic group (Fig. 2 b), which are $022-\mathrm{H} 22 \cdots \mathrm{O} 20\left(2.21 \AA, 141.9^{\circ}\right)$ and $\mathrm{C} 21-\mathrm{H} 21 \mathrm{~B} \cdots \mathrm{O} 22\left(2.65 \AA, 141.6^{\circ}\right)$. The distances are relatively long and the torsion angles are relatively low when compared with those of hydrogen bonds in the sugar backbone. It is also observed that, there is a $\mathrm{C}-\mathrm{H} \cdots n$ interaction between $\mathrm{H} 13$ to the centroid of C14-C19 with a short contact of $2.71 \AA$ (Fig. 2c).

MBS-Cinn crystallises in the space group $P 2_{1}$ (monoclinic crystal system) with neighbouring molecules in a herringbone packing motif (ESI Fig. 38). ${ }^{42}$ Crystals of MBS-Cinn diffracted weakly; the hydrogen positions of the $\mathrm{OH}$ groups were not observed in the electron density map. The hydroxyl hydrogen atoms in the model were geometrically placed to donate hydrogen bonds to the closest suitable acceptors. There are several plausible combinations of hydrogen atom positions in network of $\mathrm{OH}$ groups; the hydrogen bond positions are ambiguous and disorder cannot be discounted. The calculated positions are in ESI Table 1. Furthermore, a hydrogen bonding interaction is observed between the H20A of the aromatic group (phenyl) from the cinnamaldehyde and the O10A in the sorbitol acetal backbone ( $\mathrm{H} \cdots \mathrm{A}$ distance $2.65 \AA$ ), which propagate in one direction (Fig. $3 \mathrm{~b}$ ). The rest of the interactions are between the hydroxyl groups of the sugar backbones which are

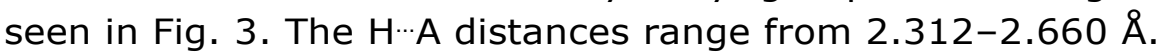


(a)

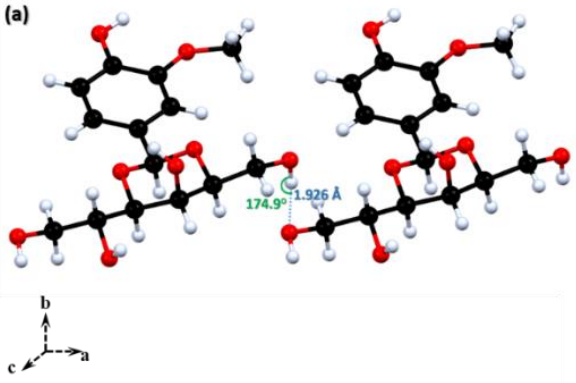

(c)

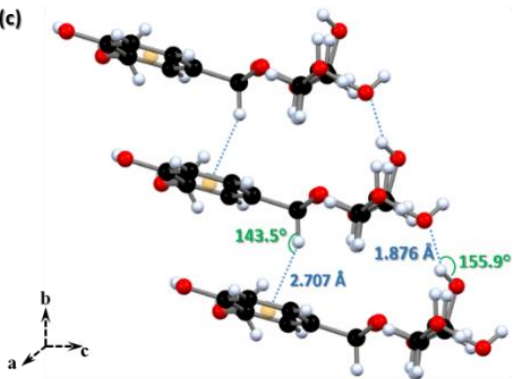

(b)

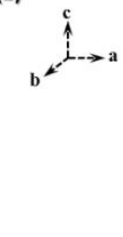

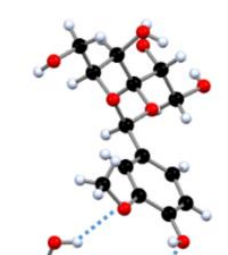
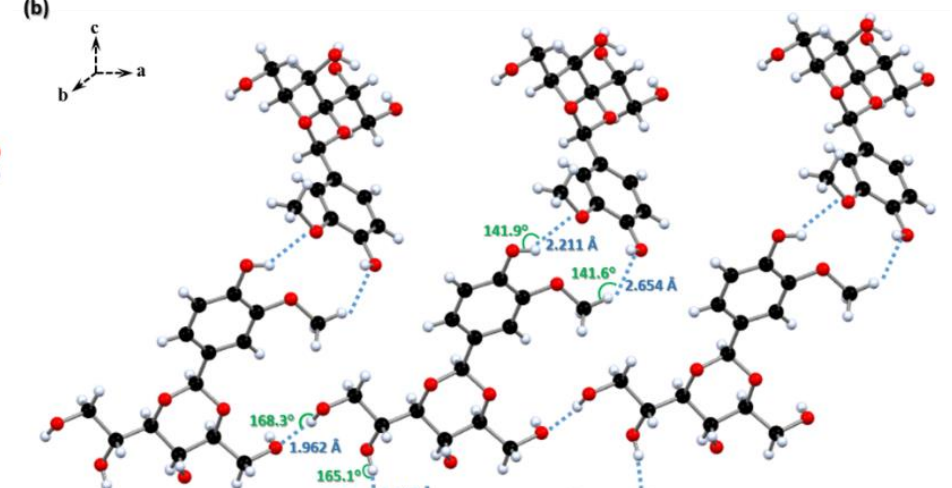

Fig. 2 | Molecular packing of MBS-Van showing different interactions between the molecules $(\mathrm{O} 2-\mathrm{H} 2 \cdots \mathrm{O} 12$ distance obtained from calculated positions).
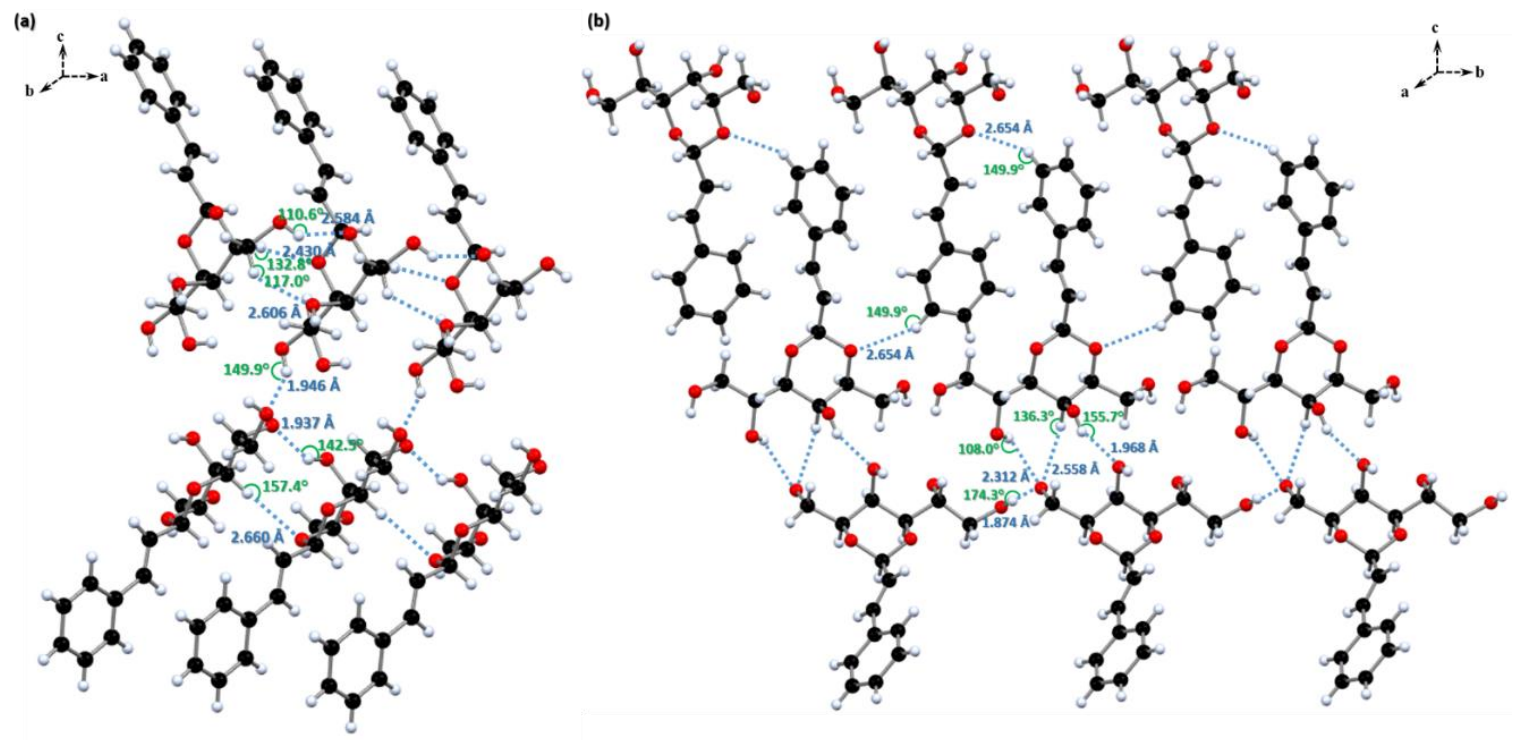

Fig. 3 | Molecular packing of MBS-Cinn showing hydrogen interactions between the molecules. (All hydrogen bonds obtained from calculated positions).

In summary, the single crystal structures of the MBS derivatives show the same general packing (Fig. 4), where hydrophilic and hydrophobic groups alternate. This organisational pattern of the mono-acetal compounds from the single X-ray crystallography is similar to molecular packing models of related compounds reported by Song et al. ${ }^{35}$ and Fan et al. ${ }^{43}$ in terms of hydrogen bonding interactions, although in our case the crystal structures show no significant $\Pi-n$ interactions. The interactions of the molecules are mainly hydrogen bonding between the sorbitol moieties. This bonding is also revealed in the FTIR spectrum of MBS-iPr in Fig. 6b. It is possible that DBS derivatives could have an analogous lamellar structure with hydrogen bonds between the sugar residues. 


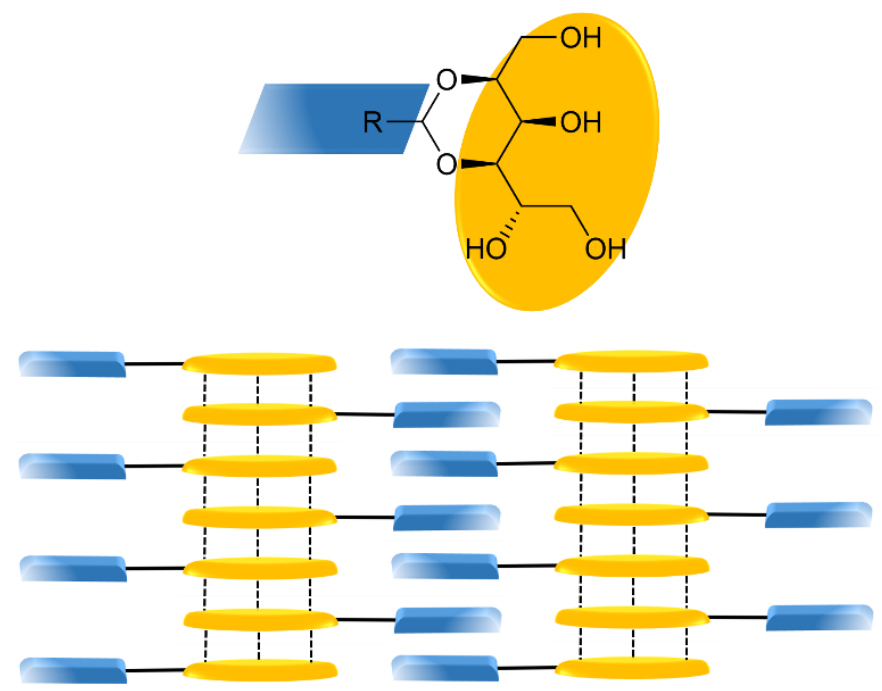

Fig. 4| Molecular packing model of mono-acetal compounds

\section{Gelation behaviour of individual gelators}

A series of gel tests were carried out on all four isolated compounds, investigating their gelation behaviour at a concentration of $10 \mathrm{mg} / \mathrm{ml}(1 \% \mathrm{w} / \mathrm{v})$. Solvents were selected to represent a broad range of different types, such as linear, cyclic, aromatic, chlorinated, alcohols, ethers, esters, and aqueous ethanolic and salt solution solutions (Table 1 and Table 2).

The dibenzylidene sorbitol derivative DBS-iPr gels a broad range of solvents, although it did not gel water because it is essentially insoluble at this concentration. Contrarily, MBSiPr gels water and aqueous salt solutions upon heating and cooling. The DBS-iPr and MBSiPr gelators further show gelation of glycerol and castor oil. Meanwhile, the remaining two mono-acetals (MBS-Cinn and MBS-Van) show no gelation ability in any of the listed organic solvents or aqueous salts tested. Rather, when soluble upon heating, they form homogeneous solutions or crystals.

The MBS compounds are largely insoluble in the apolar solvents tested, although solvents with good hydrogen bonding character (e.g. methanol) tend to solubilise them presumably because of the large amount of hydroxyl groups on the mono-acetals. The compounds were also tested in aqueous ethanolic solutions (ethanol-water mixtures), at $10 \mathrm{mg} / \mathrm{ml}$. DBS-iPr gels all the aqueous ethanol mixtures (although it is essentially insoluble in water itself) while MBS-iPr gels water and mixtures containing 10-30\% ethanol by volume. In contrast, the remaining mono-acetals displayed no gelation behaviour. MBS-Cinn is shown to be soluble in ethanol and precipitates in water, while the inverse is true for MBS-Van.

It has already been shown that certain MBS derivatives of DBS can act as hydrogelators in some salt solutions. ${ }^{35} \mathrm{~A}$ study of the effect of salt on the gelation mechanism of an MBS derivative hydrogelator showed that aqueous $\mathrm{NaCl}$ affects the morphology of the resulting xerogel and aids gelation. ${ }^{25}$

Inspired by this result, we attempted the addition of salts to MBS-iPr. Table 2 shows that MBS-iPr gels most aqueous solutions of all the salts listed, except sodium hydroxide and copper (II) chloride. The reported MBS-derived gelator, DCBS ${ }^{25}$ (DBS with 3,4 dichloro 
substituent on the benzyl ring) showed gelation in $2 \% \mathrm{NaOH}$ (aq) whilst our MBS-iPr formed a precipitate with no indication of gelation. This information indicates that the benzyl substituent has an influence on the gelation of MBS derivatives in aqueous $\mathrm{NaOH}$, although the precise reasons for this effect are unclear. On the other hand, specific coordination of $\mathrm{Cu}$ (II) by the gelator through the hydroxyl groups may have had an impact on why MBS-iPr forms a solution with $\mathrm{CuCl}_{2}$.

Table 1 | DBS-iPr and MBS-iPr gelation test in organic solvents $(1 \% \mathrm{w} / \mathrm{v})$ upon heating and cooling. $\mathrm{I}=$ insoluble, $\mathrm{G}=$ gel, $\mathrm{S}=$ solution, $\mathrm{P}=$ precipitate,$(\mathrm{T})=$ transparent,$(\mathrm{O})=$ opaque.

\begin{tabular}{|c|c|c|c|c|}
\hline Solvent & DBS-iPr & MBS-iPr & MBS-Cinn & MBS-Van \\
\hline Hexane & I & I & I & I \\
\hline Cyclohexane & G (O) & $\mathrm{P}$ & $\mathrm{P}$ & I \\
\hline Toluene & $G(T)$ & $\mathrm{P}$ & I & $P$ \\
\hline Chloroform & $G(T)$ & I & I & I \\
\hline Dichloromethane & $G(T)$ & I & I & I \\
\hline Tetrahydrofuran & $\mathrm{s}$ & I & $\mathrm{P}$ & I \\
\hline 2-Butanone & $\mathrm{S}$ & $\mathrm{P}$ & $\mathrm{P}$ & $\mathrm{P}$ \\
\hline Ethyl acetate & $G(T)$ & $\mathrm{P}$ & I & $\mathrm{P}$ \\
\hline Acetonitrile & $\mathrm{s}$ & $\mathrm{P}$ & $\mathrm{P}$ & $\mathrm{P}$ \\
\hline Isopropranol & $G(T)$ & $\mathrm{S}$ & $P$ & I \\
\hline Methanol & G (O) & $\mathrm{S}$ & $\mathrm{P}$ & $\mathrm{S}$ \\
\hline Ethanol & G (O) & $\mathrm{s}$ & $\mathrm{s}$ & $\mathrm{P}$ \\
\hline $90: 10$ & G (O) & $\mathrm{s}$ & $\mathrm{s}$ & $\mathrm{P}$ \\
\hline $80: 20$ & $\mathrm{G}(\mathrm{O})$ & $\mathrm{s}$ & $\mathrm{s}$ & $\mathrm{s}$ \\
\hline $70: 30$ & $G(0)$ & $\mathrm{S}$ & $\mathrm{S}$ & $\mathrm{s}$ \\
\hline $60: 40$ & $G(0)$ & $\mathrm{S}$ & $\mathrm{S}$ & $\mathrm{S}$ \\
\hline $50: 50$ & G (O) & $\mathrm{S}$ & $\mathrm{s}$ & $\mathrm{S}$ \\
\hline $40: 60$ & G (O) & $\mathrm{s}$ & $\mathrm{s}$ & $\mathrm{S}$ \\
\hline $30: 70$ & $\mathrm{G}(\mathrm{O})$ & $\mathrm{G}(\mathrm{O})$ & $\mathrm{P}$ & $\mathrm{s}$ \\
\hline $20: 80$ & $\mathrm{G}(0)$ & G (O) & $\mathrm{P}$ & $\mathrm{S}$ \\
\hline $10: 90$ & G (O) & G (O) & $P$ & $\mathrm{~S}$ \\
\hline Water & I & G (O) & $P$ & $\mathrm{~S}$ \\
\hline Glycerol & $\mathrm{G}(\mathrm{O})$ & $G(T)$ & $\mathrm{s}$ & $\mathrm{s}$ \\
\hline Castor Oil & G (O) & G (O) & $\mathrm{S}$ & $\mathrm{s}$ \\
\hline
\end{tabular}

Table 2 | Synthesised mono-acetals in salt solutions $(1 \% \mathrm{w} / \mathrm{v})$ upon heating and cooling. $\mathrm{S}=$ solution and $\mathrm{P}=$ precipitate, $\mathrm{G}=$ gel, $(\mathrm{T})=$ transparent, $(\mathrm{O})=$ opaque

\begin{tabular}{c|ccc}
$\begin{array}{c}\text { Salt Solution } \\
(2 \% \mathrm{~W} / \mathrm{V})\end{array}$ & MBS-Cinn & MBS-Van & $\mathrm{MBS}-i \mathrm{Pr}$ \\
\hline $\mathrm{NaCl}$ & $\mathrm{P}$ & $\mathrm{S}$ & $\mathrm{G}(0)$ \\
$\mathrm{KCl}$ & $\mathrm{P}$ & $\mathrm{S}$ & $\mathrm{G}(0)$ \\
$\mathrm{LiCl}$ & $\mathrm{P}$ & $\mathrm{S}$ & $\mathrm{G}(0)$ \\
$\mathrm{ZnCl}_{2}$ & $\mathrm{P}$ & $\mathrm{S}$ & $\mathrm{G}(0)$ \\
$\mathrm{CaCl}_{2}$ & $\mathrm{P}$ & $\mathrm{S}$ & $\mathrm{G}(0)$ \\
$\mathrm{MgCl}_{2}$ & $\mathrm{P}$ & $\mathrm{S}$ & $\mathrm{G}(\mathrm{O})$ \\
$\mathrm{CCCl}_{2}$ & $\mathrm{P}$ & $\mathrm{S}$ & $\mathrm{S}$ \\
$\mathrm{NaOH}_{\mathrm{Na}_{2} \mathrm{SO}_{4}}$ & $\mathrm{P}$ & $\mathrm{S}$ & $\mathrm{P}$ \\
& $\mathrm{P}$ & $\mathrm{S}$ & $\mathrm{G}(0)$
\end{tabular}

Also, gelation test of mono-acetals MBS-Cinn and MBS-Van were performed, but they do not show any gelation even in the presence of salts (Table 2). MBS-Van forms solutions at $10 \mathrm{mg} / \mathrm{ml}$ concentration but precipitates as fibrous, needle-like crystals at $20 \mathrm{mg} / \mathrm{ml}$ concentrations. On the other hand, MBS-Cinn produces similar needle-like crystals at 10 
$\mathrm{mg} / \mathrm{ml}$. These results are in line with the previous series of tests, which showed that MBSCinn precipitates from water at the stated concentrations while MBS-Van is soluble.

Neither of them gelates in any organic solvents and aqueous salt tested. Solubility is a major factor, because MBS-Cinn is shown to be soluble in ethanol and precipitates in water with the inverse being true to MBS-Van. In addition, another possible factor is the functionality offered by these acetal substituents and the lack of solubilising bulky substituents. The acetal substituent of MBS-iPr has the most steric bulk and MBS-Cinn has the least. It is possible that the bulkier the substituent is on the acetal group, the higher the chance it will self-assemble and exhibit gelation properties.

The differences in the gelation properties of DBS-iPr and MBS-iPr are apparent where the former is insoluble and the later forms a gel in water. For this reason, phase diagrams for DBS-iPr and MBS-iPr were carried out in aqueous ethanolic mixtures (see ESI Fig. 29). The phase diagrams show that DBS-iPr displays significant gelation properties since it forms gels in aqueous ethanolic mixtures at different concentrations as low as $1 \mathrm{mg} / \mathrm{mL}$. The diagrams further show that DBS-iPr gelates in ethanol at $7 \mathrm{mg} / \mathrm{mL}$ and precipitates at $5 \mathrm{mg} / \mathrm{mL}$. Because of its insolubility, no gelation was observed for DBS-iPr in water regardless of the concentration. Conversely, MBS-iPr gelates in water at different concentrations and as low as $7 \mathrm{mg} / \mathrm{mL}$, forming a partial gel at $5 \mathrm{mg} / \mathrm{mL}$.

\section{Gelation behaviour of DBS and MBS-iPr mixture}

Given the array of dissolution profiles between the mono and the diacetal compounds reported here in ethanol-water mixtures, it seemed fitting to mix the gelators and analyse their combined gelation phase diagram in ethanol-water mixtures. The inspiration for this work came from research performed by Fan and colleagues ${ }^{43}$ who demonstrated the tunability of self-assembly of two-component gels from donor and acceptor MBS derivatives, where dual component material behaviour was observed. ${ }^{44}$ Their experiments were focused on different organic solvents and inferred a $n$-donor-acceptor interaction as the driving force for gelation. Here we focus on the full range of water-ethanol compositions, because the extremes of composition are only gelled by one of the components.

Equimolar amounts of DBS-iPr and MBS-iPr were placed in a vial, aqueous ethanolic solutions with the appropriate ratios were added afterwards. Gelation properties were tested upon heating (until the mixture was completely transparent by eye) and cooling. Fig. 5 shows that gelation was observed between $7-15 \mathrm{mg} / \mathrm{mL}$ of equimolar amounts of DBS-iPr and MBS-iPr in $100 \%$ ethanol, $100 \%$ water and all aqueous ethanolic solution ratios in between (lowest concentration consisting $4.1 \mathrm{mg}$ and $2.9 \mathrm{mg}$ of DBS-iPr and MBS$i \operatorname{Pr}$, respectively). The phase diagram of DBS-iPr (ESI Fig. 29) has a similar trend with the equimolar DBS-MBS-iPr phase diagram (Fig. 5). The obvious difference to the phase diagram of DBS-iPr is that the gelation extended into water. Also, considering only the concentration of the DBS-iPr component, the phase diagram is shifted to lower concentration. Overall, this phase diagram shows that MBS-iPr has a positive influence on the gelation of DBS-iPr. 


\begin{tabular}{|c|c|c|c|c|c|c|}
\hline \multirow{11}{*}{ 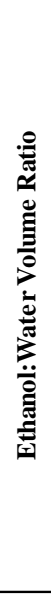 } & $\mathrm{EtOH}$ & S & $\mathrm{P}$ & $\mathrm{G}(\mathrm{O})$ & $\mathrm{G}(\mathrm{O})$ & $\mathrm{G}(\mathrm{O})$ \\
\hline & $90: 10$ & S & $\mathrm{P}$ & $\mathrm{G}(\mathrm{O})$ & $\mathrm{G}(\mathrm{O})$ & $\mathrm{G}(\mathrm{O})$ \\
\hline & $80: 20$ & $S$ & $\mathrm{G}(\mathrm{O})^{\mathrm{b}}$ & $\mathrm{G}(\mathrm{O})$ & $\mathrm{G}(\mathrm{O})$ & $\mathrm{G}(\mathrm{O})$ \\
\hline & $70: 30$ & $\mathrm{G}(\mathrm{O})^{\mathrm{b}}$ & $\mathrm{G}(\mathrm{O})$ & $\mathrm{G}(\mathrm{O})$ & $\mathrm{G}(\mathrm{O})$ & $\mathrm{G}(\mathrm{O})$ \\
\hline & $60: 40$ & $\mathrm{G}(\mathrm{O})^{\mathrm{c}}$ & $\mathrm{G}(\mathrm{O})$ & $\mathrm{G}(\mathrm{O})$ & $\mathrm{G}(\mathrm{O})$ & $\mathrm{G}(\mathrm{O})$ \\
\hline & $50: 50$ & $\mathrm{G}(\mathrm{O})^{\mathrm{c}}$ & $\mathrm{G}(\mathrm{T})$ & $\mathrm{G}(\mathrm{O})$ & $\mathrm{G}(\mathrm{O})$ & $\mathrm{G}(\mathrm{O})$ \\
\hline & $40: 60$ & $\mathrm{G}(\mathrm{O})$ & $\mathrm{G}(\mathrm{O})$ & $\mathrm{G}(\mathrm{O})$ & $\mathrm{G}(\mathrm{O})$ & $\mathrm{G}(\mathrm{O})$ \\
\hline & $30: 70$ & $\mathrm{G}(\mathrm{O})$ & $\mathrm{G}(\mathrm{O})$ & $\mathrm{G}(\mathrm{O})$ & $\mathrm{G}(\mathrm{O})$ & $\mathrm{G}(\mathrm{O})$ \\
\hline & $20: 80$ & $\mathrm{G}(\mathrm{O})$ & $\mathrm{G}(\mathrm{O})$ & $\mathrm{G}(\mathrm{O})$ & $\mathrm{G}(\mathrm{O})$ & $\mathrm{G}(\mathrm{O})$ \\
\hline & $10: 90$ & $\mathrm{G}(\mathrm{T})^{\mathrm{a}}$ & $\mathrm{G}(\mathrm{O})^{\mathrm{a}}$ & $\mathrm{G}(\mathrm{O})^{\mathrm{a}}$ & $\mathrm{G}(\mathrm{O})^{\mathrm{a}}$ & $\mathrm{G}(\mathrm{O})^{\mathrm{a}}$ \\
\hline & $\mathrm{H}_{2} \mathrm{O}$ & I & I & $\mathrm{G}(\mathrm{T})^{\mathrm{a}}$ & $\mathrm{G}(\mathrm{O})^{\mathrm{a}}$ & $\mathrm{G}(\mathrm{O})^{\mathrm{a}}$ \\
\hline & & $3 \mathrm{mg}$ & $5 \mathrm{mg}$ & $7 \mathrm{mg}$ & $10 \mathrm{mg}$ & $15 \mathrm{mg}$ \\
\hline & & $(1.8: 1.2) \mathrm{mg}$ & $(2.9: 2.1) \mathrm{mg}$ & (4.1:2.9) mg & $(5.9: 4.1) \mathrm{mg}$ & (8.8:6.2) mg \\
\hline & & Con & ration of equ & olar DBS-iPI & nd MBS-iPr & $\mathrm{g} / \mathrm{mL})$ \\
\hline
\end{tabular}

Fig. 5 | Phase diagram of equimolar ratio of DBS-iPr and MBS-iPr upon heating and cooling. $\mathrm{G}=$ gel, $\mathrm{S}=$ solution, $\mathrm{I}=$ insoluble, $\mathrm{P}=$ precipitate, $(\mathrm{T})$ =transparent, $(\mathrm{O})=$ opaque. ${ }^{\text {anot }}$ all solid dissolved, ${ }^{\mathrm{b}}$ gelation occurred overnight and ctransparent to opaque gel occurred overnight at $25^{\circ} \mathrm{C}$.

\section{Infrared Spectroscopy}

Given that both gelators efficiently immobilise polar solvents such as water and ethanol, we considered that hydrogen bonding might be playing an important role in the formation of the gels. FTIR was used to investigate the effect of these solvents on the gel structure focusing on both intermolecular and intramolecular interactions between $-\mathrm{OH}$ groups that might contribute to the stabilisation of the self-assembled aggregates. ${ }^{22}$

IR spectra of MBS-iPr and DBS-iPr as crystalline powders, gel and xerogels were measured, and the results are shown in Fig. 6. Lai et al., ${ }^{45}$ reported that according to the IR handbook ${ }^{46}$, the intermolecular hydrogen bonds for the $\mathrm{OH}$ groups appear in the range of $3200-3550 \mathrm{~cm}^{-1}$, and the intramolecular hydrogen bonds appear in the range of 3400$3590 \mathrm{~cm}^{-1}$. For all samples, peaks at approximately $3250-3350 \mathrm{~cm}^{-1}$ were observed which are assigned to the intermolecular hydrogen bonding. This observation indicates that intermolecular hydrogen bonding between the $-\mathrm{OH}$ groups in the molecule is one of the driving forces for self-assembly of DBS-iPr and MBS-iPr. It is noteworthy that for the crystals of the MBS compounds derived from vanillin and cinnamaldehyde (that do not form gels) the IR spectra between 3200 and $3400 \mathrm{~cm}^{-1}$ are very similar to the xerogel of MBS-iPr (see ESI), indicating that the hydrogen bonding is similar in all the MBS compounds, whether in crystal or gel form.

Furthermore, the IR spectra for MBS-iPr show no change in wavenumber between the xerogel, wet gel and the crystalline powder (Fig. 6b). In contrast, the DBS-iPr crystalline spectrum shows peaks at 2956 and $3267 \mathrm{~cm}^{-1}$ (Fig. 6a); these peaks were seen to have shifted to higher wavenumbers in the xerogel and wet gel spectra. These observations indicate that the hydrogen bonding between the $-\mathrm{OH}$ groups are being modified by the introduction of ethanol. The spectra show that DBS-iPr in the crystalline state has stronger hydrogen bonds in comparison to that in DBS-iPr gel in ethanol and its xerogel. Furthermore, the polymeric hydrogen bond peak was reported by Liddel and Becker ${ }^{47}$ to be near $3350 \mathrm{~cm}^{-1}$ whereas the dimer band is found near $3500 \mathrm{~cm}^{-1}$. As DBS-iPr xerogel 
and wet gel in ethanol showed peaks at $3347 \mathrm{~cm}^{-1}$ and $3349 \mathrm{~cm}^{-1}$, respectively, we suggest that DBS-iPr self-assembles into a polymeric structure via hydrogen bond chains.

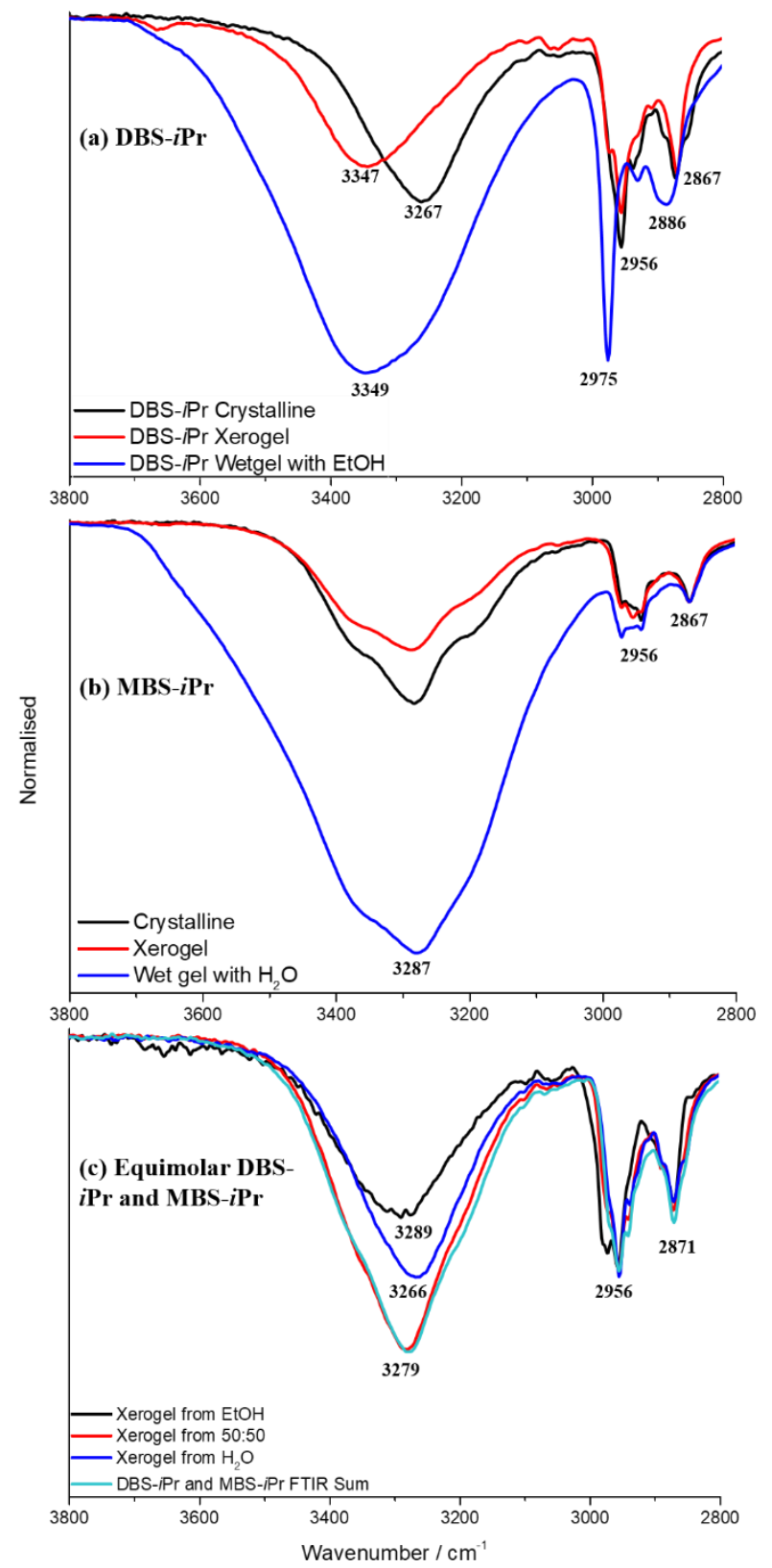

Fig. 6| IR spectra of crystalline powder, xerogels and gels: (a) DBS-iPr and (b) MBS-iPr. (c) Xerogels of equimolar DBS-iPr and MBS-iPr. Gels were formed with respective solvents: EtOH for DBS and water for MBS and the xerogels are air dried gels. Data were normalised.

It is apparent that the wet gels have the strongest intensities at around $3250-3350 \mathrm{~cm}^{-}$ ${ }^{1}$ because of the strong hydrogen bonding interactions between (i) the solvent molecules, (ii) the gelator molecules and (iii) both the solvent and gelator molecules. As xerogels have the weakest intensities, we believe that the intermolecular interactions between the solvent molecules contribute greatly to the intensity of the damp gel. Nevertheless, the intensities of the xerogels imply that the dipole moment of the stretch in the xerogel state is weaker than the dipole moment in the crystalline state. ${ }^{48}$

The sum of the IR data of DBS-iPr and MBS-iPr was calculated and plotted with the FTIR spectra of equimolar DBS-iPr and MBS-iPr in (i) ethanol, (ii) 50:50 ethanol-water and (iii) 
water (Fig. 6c). It shows that the IR spectra of the xerogels resemble the sum of the spectra of their individual components, meaning that the interactions present in the pure compounds are also found in the equimolar xerogels and indicate self-sorting. Should a co-assembly take place, in which the MBS and DBS compounds are hypothetically incorporated in the same lamellae in the fibres, one would expect a significantly different IR signature. Furthermore, only the equimolar xerogel from 50:50 ethanol-water has the same intensity as the FTIR sum at the $-\mathrm{OH}$ stretch at $3279 \mathrm{~cm}^{-1}$. It was also observed that the higher the ethanol content of the solvent, the higher the frequency of the -OH stretch. This observation suggests that xerogels from ethanol have weaker hydrogen bonds in comparison to xerogels from water ${ }^{49}$ possibly because water is a better hydrogen bond donor than ethanol.

\section{Xerogel Morphologies}

The difference in morphology between the mono- and di-benzylidene sorbitol xerogels was investigated by scanning electron microscopy (SEM). Samples were prepared by drying MBS-iPr and DBS-iPr gels obtained from the various solvents and mixtures on a SEM stub in vacuo followed by iridium coating under vacuum. While the drying process causes collapse of the gel, and could result in some dissolved material precipitating during drying, the results do indicate significant differences between the samples that are surely a result of the initial gel structure in the solvent. However, caution is recommended in interpretation because the drying of samples with different liquid compositions could result in contrasting drying times and fibre coalescence, and it is likely that the observed dimensions in the SEM images are larger than those of the solvated fibres in the gels. ${ }^{50}$

SEM micrographs of DBS-iPr, shown in Fig. 7, comprise different xerogel morphologies depending on the solvent medium. The DBS-iPr fibres remaining from the ethanol gel (Fig. 7a) are relatively wider than the fibres grown from evaporation of 70:30 ethanol-water from the gel (Fig. 7b). They do, however, show similar morphology of a ribbon-like structure. On the other hand, the fibres formed from evaporation of the toluene gel are much thinner than those formed in ethanol ( $31 \pm 8 \mathrm{~nm}$ vs $700 \pm 4 \mathrm{~nm}$ width as seen in Fig. 7a and c). This difference is probably a result of the differing solubility of DBS-iPr in the two solvents, with the gelator being more soluble (and therefore better solvated) in toluene than ethanol. Furthermore, the difference is easily observable on the macroscale. Gels in toluene and most of the organic solvents are transparent while the gels in ethanol, ethanol-water solutions, methanol and cyclohexane are opaque. The effect of gel fibre size on gel transparency is therefore quite apparent. SEM micrographs were also taken for the DBS-iPr xerogel formed from $1 \mathrm{mg} / \mathrm{mL}$ in 50:50 ethanol-water (ESI Fig. 30b). The morphology is dissimilar to the fibres in the xerogel formed from ethanol. 

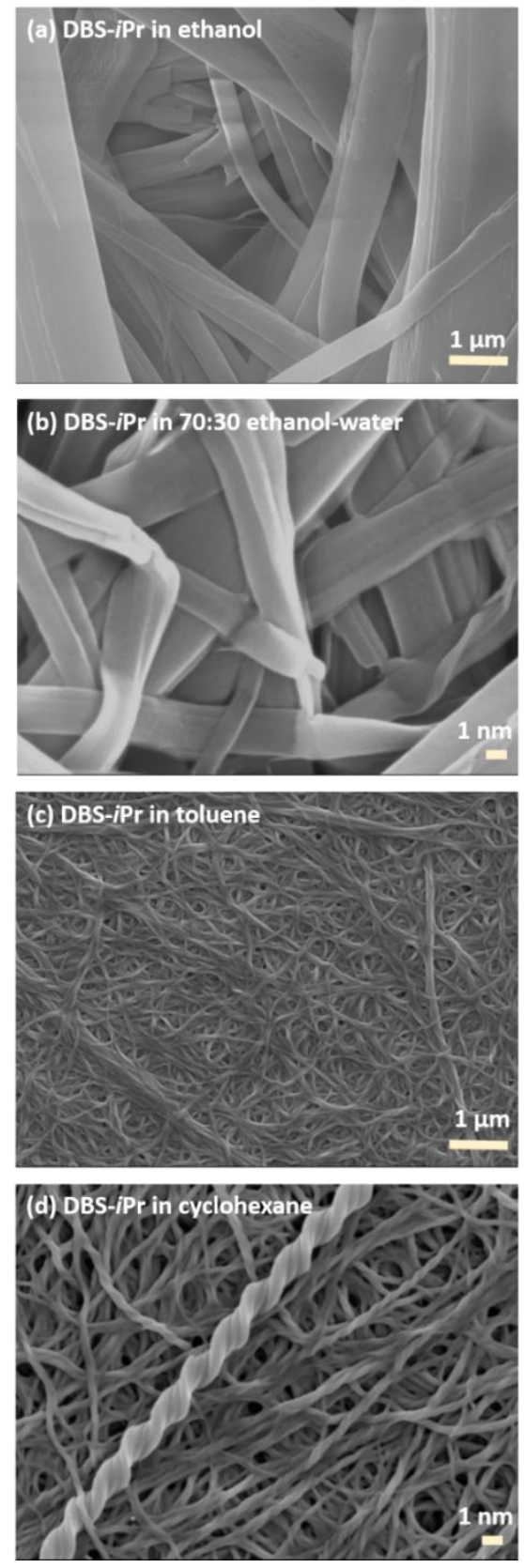

Fig. 7 | SEM micrographs of DBS-iPr xerogels formed by $1 \% \mathrm{w} / \mathrm{v}$. Conditions: xerogel was prepared by drying the gel in air and then coating with $5 \mathrm{~nm}$ Ir before imaging under vacuum at 5kV. Scale bar represents: $1 \mu \mathrm{m}$ in $\mathrm{a}$ and $\mathrm{c} ; 100 \mathrm{~nm}$ in $\mathrm{b}$ and $\mathrm{d}$.

Only the xerogel from cyclohexane shows helical fibres in different sizes consistently (Fig. $7 d$ ). This finding is in accordance with that of Song and colleagues ${ }^{24}$, who described how polar solvents (such as $i \mathrm{PA}$, water and ethanol) discourage helical fibre formation and lead to smooth, straight structure. On the other hand, non-polar solvents can induce twisting in the chiral assemblies as the gelator self-assembled with strong hydrogen bonding interactions. ${ }^{24}$

The helical fibres in xerogel formed from cyclohexane have an average width of $42 \pm 5$ $\mathrm{nm}$. All of the DBS-iPr fibres in this material show anti-clockwise twisting. A plot of full twist period ( $2 \mathrm{P}$ or pitch) against the smallest fibre size in cross section ( $h$ or minimum width) is given in Fig. 8. 


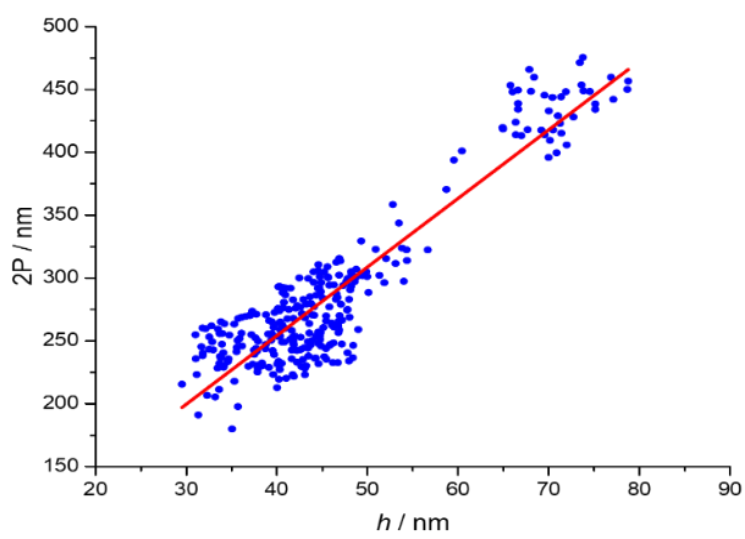

Fig. 8 | Correlation between full twist period $(2 \mathrm{P}, 2 \mathrm{n}$ rotation, $\mathrm{nm}$ ) and the smallest fibre size in cross section ( $h$, $\mathrm{nm}$ ) of DBS-iPr twisted fibres in cyclohexane upon heating and cooling

It has been reported that as twisted fibres narrow, the pitch changes sharply. ${ }^{51}$ Furthermore, Shtukenberg et al. ${ }^{52}$ reported that for all twisted crystals in the size range from $\mathrm{nm}$ to $\mathrm{cm}$, twist period is proportional to the cross section size. This effect is observed as a positive correlation is obtained between the pitch and the minimum width. It has been shown that strain induces the twisting of fibres and for an elastically twisted fibre the maximum strain $\left(\gamma_{\max }\right)$ forms on the outer surface and approaches $\gamma_{\max }=\pi h /(2 \mathrm{P}) .{ }^{53} \mathrm{In}$ addition, the curvature radius, $R$, could also be obtained from $Y \max =h /(2 R)$. With the average of $287 \mathrm{~nm}$ for the pitch and $46 \mathrm{~nm}$ for the minimum width, a strain of 0.500 and a curvature radius of $45.7 \mathrm{~nm}$ were calculated. The strain value indicates that the fibres formed in cyclohexane have elastic properties.

There is a common thread for the morphology of the fibres between the xerogels formed from ethanol-water, water and aqueous salt solutions present in the MBS-iPr. The majority of the fibres present from ethanol-water mixtures (ESI Fig. $30 \mathrm{~g}-\mathrm{i}$ ) and aqueous salt solutions (Fig. 9b and ESI Fig. 30d and f) are similar to the morphology of MBS-iPr xerogel in water (Fig. 9a). They are all quite thick tape-like fibres. Furthermore, the xerogels of MBS-iPr from a salt solution of $\mathrm{Na}_{2} \mathrm{SO}_{4}$ (Fig. 9b) with an average width of $170 \pm 4 \mathrm{~nm}$, and 10:90 ethanol-water solution (ESI Fig. 29i) with an average width of $77 \pm 6 \mathrm{~nm}$, are very similar where distorted rod-like fibres intertwine with smaller fibres. As for the xerogel formed from $\mathrm{CaCl}_{2}$ solution, the morphology appears to be different from the majority of the gels (Fig. 9c). The fibres are a mixture of clustered and individual fibres with an average width of $340 \pm 3 \mathrm{~nm}$. The gel formed in $\mathrm{ZnCl}_{2}$ solution exhibit webbed fibres connected to each other with an average width of $650 \pm 3 \mathrm{~nm}$. All MBS-iPr gels are opaque, it is useful to see the difference in the microstructure of the MBS-iPr xerogels to see the effect of aqueous salt solutions on the change of morphology, which is correlated with the mechanical properties of the materials (see below). 

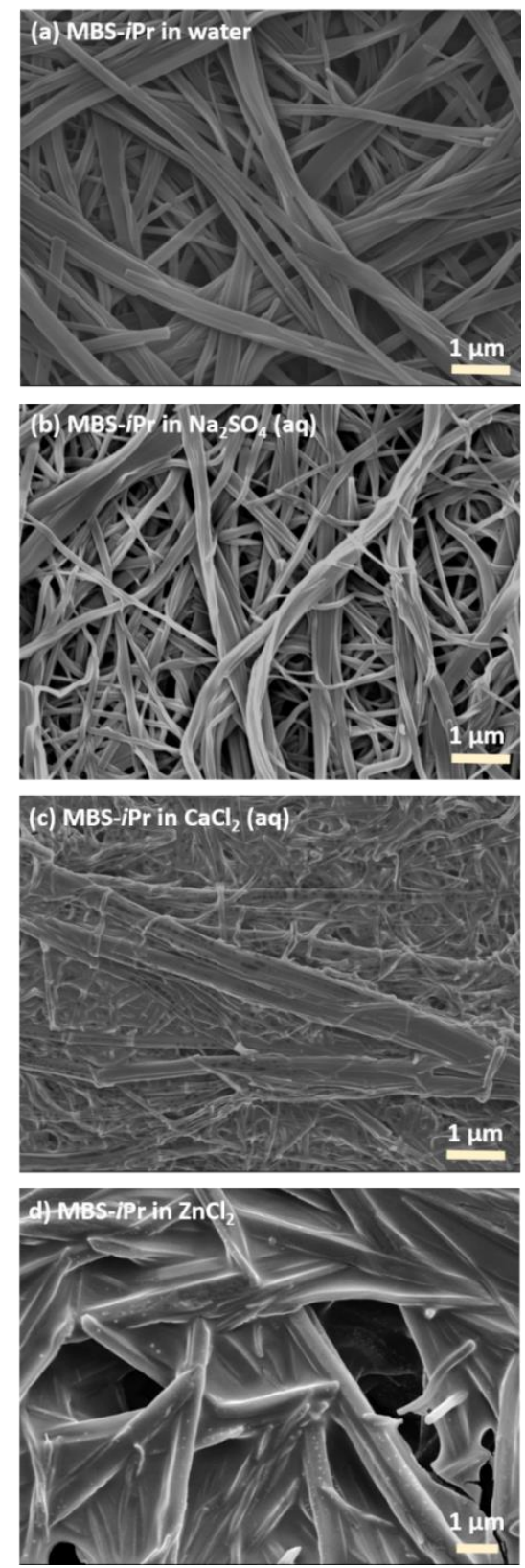

Fig. 9 | SEM micrographs of MBS-iPr xerogels formed by $1 \% \mathrm{w} / \mathrm{v}$ in $2 \% \mathrm{w} / \mathrm{v}$ aqueous salt solutions. Conditions: xerogel was prepared by drying the gel in air and then coating with $5 \mathrm{~nm}$ Ir before imaging under vacuum at $5 \mathrm{kV}$. Scale bars represent $1 \mu \mathrm{m}$.

SEM imaging was also done for the equimolar xerogels of DBS-iPr and MBS-iPr to investigate their morphology (Fig. 10 and ESI Fig. 33). In pure solvents (water and ethanol), a mixture of thick and thin fibres were seen. A clear bimodal distribution of widths is observed. The thin fibres in both solvents exhibit similar average width as seen in Fig. 11 and Table 3, whereas the thick fibres are quite different. 

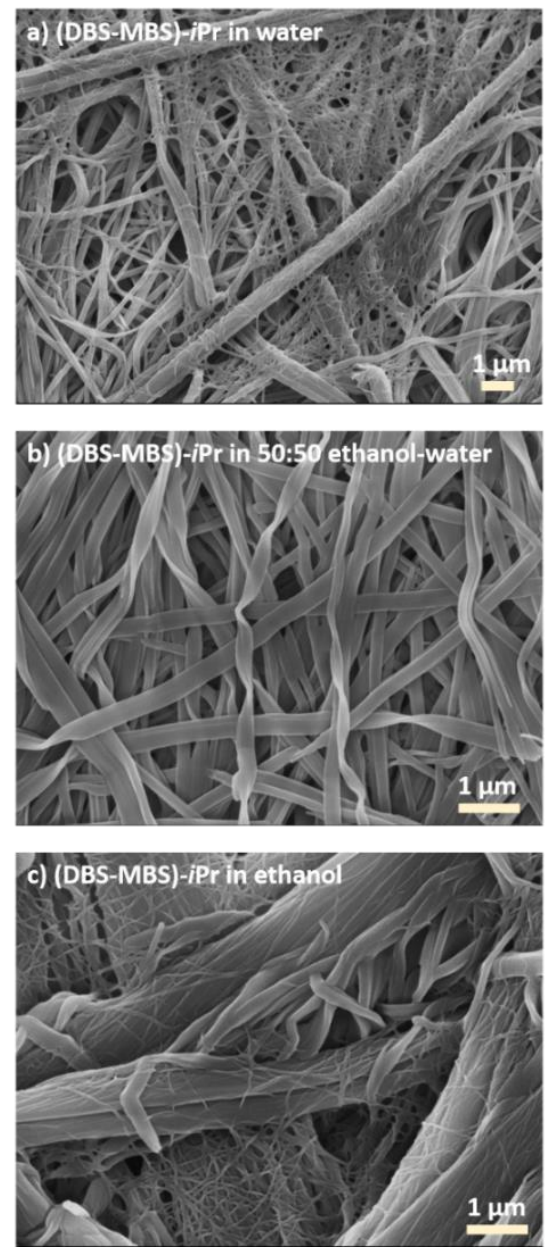

Fig. 10 SEM micrographs of dried xerogel formed by equimolar $1 \% \mathrm{w} / \mathrm{v}$ DBS-iPr and MBS-iPr upon heating and cooling. Conditions: xerogel prepared by drying the gel in air and then coating with $5 \mathrm{~nm}$ Ir before imaging under vacuum at $5 \mathrm{kV}$. Scale bar in all images represents $1 \mu \mathrm{m}$.

Thick fibres of the equimolar xerogel in water (Fig. 10a) resemble the MBS-iPr xerogel in water (Fig. 9a) having fibre widths of $260 \pm 4 \mathrm{~nm}$ and $190 \pm 3 \mathrm{~nm}$, respectively. On the other hand, the equimolar xerogel in ethanol (Fig. 10c), which also displays a bimodal distribution of fibre width (Fig. 11c), appears to have root-like fibres tangling around the thick fibres with an average fibre width of $590 \pm 20 \mathrm{~nm}$ for the thick fibres and $21 \pm 1 \mathrm{~nm}$ for the thin ones. Although the morphology and the fibre width is not similar to DBS-iPr in ethanol (700 $\pm 4 \mathrm{~nm}$, Fig. 7a), both possess the highest fibre width. As a result of this inspection, we anticipate that the thick fibres in water and ethanol correspond to MBS-iPr and DBS-iPr, respectively, whilst the thinner fibres are other component which could partially precipitate during the process. Yet they clearly have an influence on gel properties. Remarkably, (DBS-MBS)-iPr xerogel formed in 50:50 ethanol-water solution (where individual MBS-iPr did not form a gel at this concentration) only contain uniformsized fibres (Fig. 10b) with an average width of $190 \pm 4 \mathrm{~nm}$. On the other hand, the DBS$i \operatorname{Pr}$ xerogel formed in the same solution has fibres with an average width of $89 \pm 10 \mathrm{~nm}$ (ESI Fig. 30). 
Table 3 | Fibre average width for xerogels made from DBS-iPr and MBS-iPr gels at $10 \mathrm{mg} / \mathrm{mL}$. ${ }^{\text {axerogel }}$ made from $1 \mathrm{mg} / \mathrm{mL}$

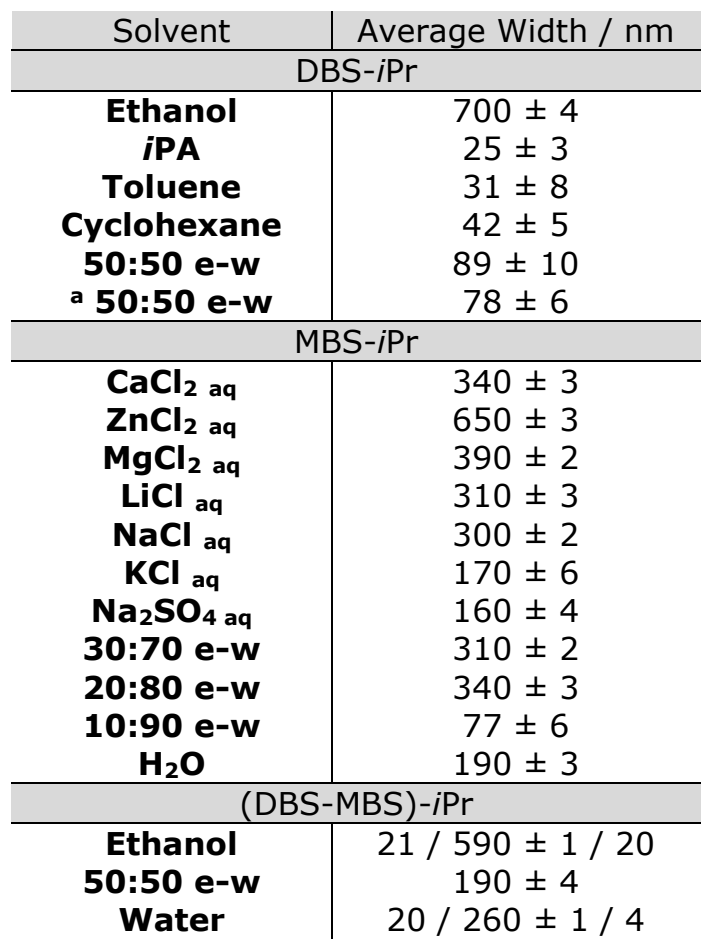

All SEM micrographs of the xerogels revealed that DBS-iPr and MBS-iPr form fibrous networks, in common with other sorbitol xerogels. ${ }^{11,21,54,55}$ We show that the nature of the networks in the solids remaining after solvent evaporation depend on the liquid and the presence of salts in the case of hydrogels. While caution is advised in the interpretation of SEM textures from dried gels, exemplified by the work of Mears et al. ${ }^{56}$ where drying can affect significantly the fibre network, we believe that the dramatic differences seen between morphologies of xerogels from the same solvents in the present case at least provide a strong indication of large differences in gel structure.

In the present case, the chiral nature of the compounds might have aided characterisation of the gels in the presence of the immobilised solvent. Li et al. ${ }^{24}$ studied the chiral structure of the aggregate in a gel using circular dichroism (CD) spectroscopy. Comparison of the morphologies of the xerogels with the CD spectra of the wet gels could have provided useful information. In our hands, this has not been possible because of the high concentrations required and the light scattering by the samples. For example, 10 $\mathrm{mg} / \mathrm{mL}$ DBS-iPr in cyclohexane would be a good example to see how the twisted helical fibres behave in circularly polarised light. The DBS-iPr powder is difficult to dissolve in cyclohexane and must be heated up to boiling until no solid is seen by eye. The rapid formation of the gels during cooling makes it difficult to transfer the solution into a cuvette and therefore, the attempt to obtain a CD spectra for DBS-iPr in cyclohexane was challenging. Furthermore, the opaqueness of the gels, and their effective light scattering because of the fibre dimensions observed by SEM for both MBS-iPr and DBS-iPr, make CD spectra very difficult to obtain, and in any case irreproducible and unreliable in our hands. 
a) (DBS-MBS)-iPr in water

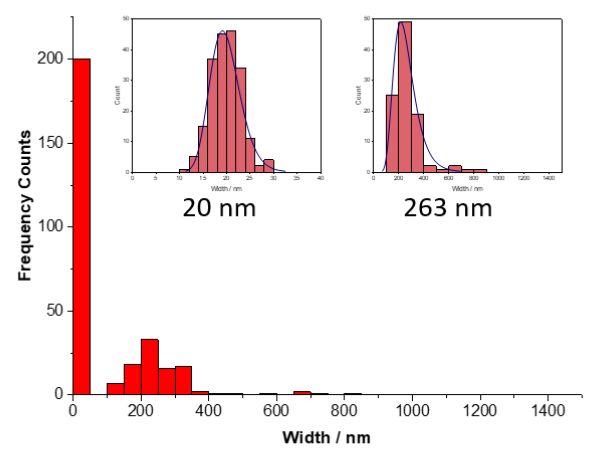

b) (DBS-MBS)-iPr in 50:50 ethanol-water

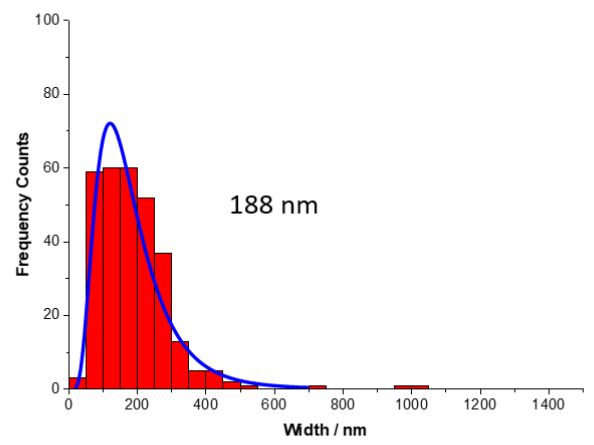

c) (DBS-MBS)-iPr in ethanol

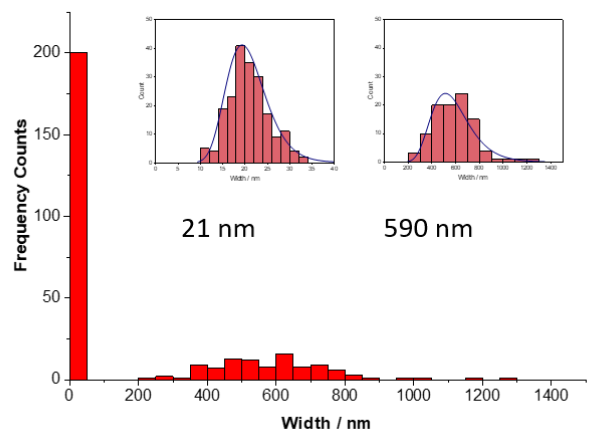

Fig. 11 Distribution histograms of fibres widths for xerogels formed from equimolar (DBS-MBS)-iPr in (a) water, (b) 50:50 ethanol-water and (c) ethanol.

\section{Gel Rheology}

Rheological data were collected for DBS-iPr gels in dichloromethane (DCM) and iso-propyl alcohol (iPA), and MBS-iPr samples formed in water and salt solutions. Oscillatory measurements were conducted on samples of the gels by applying strain deformation to the sample and measuring the stress response while keeping the frequency at a constant value of $1 \mathrm{~Hz}$. This method allows determination of their storage and loss moduli ( $\mathrm{G}^{\prime}$ and G" respectively) across a range of applied strain (Fig. 12, Fig. 13, Fig. 14). Materials exhibit elastic properties when $\mathrm{G}^{\prime}>\mathrm{G}^{\prime \prime}$, viscoelastic properties when the two values are equal, and viscous properties when $\mathrm{G}^{\prime}<\mathrm{G}^{\prime \prime} .{ }^{57}$ Elastic and viscous in this context mean elastic solid and viscous liquid. Therefore, the crossing point on a graph where storage and loss moduli are plotted against strain will show the point where strain causes the gel to flow like a Newtonian liquid. ${ }^{58}$ 
We can see that the two DBS-iPr gels exhibit different strain percentages (the DCM gel at $3 \%$ strain, the iPA gel at 6\%) and that the $G^{\prime}$ and $G^{\prime \prime}$ values for the iPA gel are approximately an order of magnitude greater than those for the DCM gel.

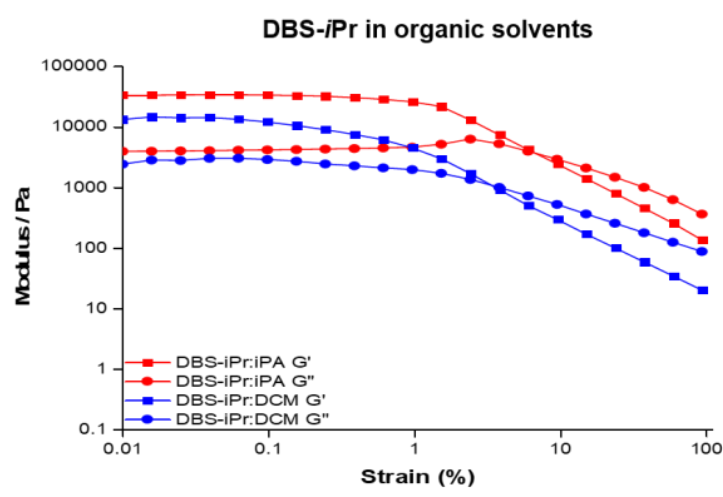

Fig. 12 | Amplitude sweep rheological data of DBS-iPr gels formed from IPA and DCM formed at $1.5 \% \mathrm{w} / \mathrm{v}$ upon heating and cooling. $\mathrm{G}^{\prime}$ as Storage Modulus and G" as Loss Modulus.
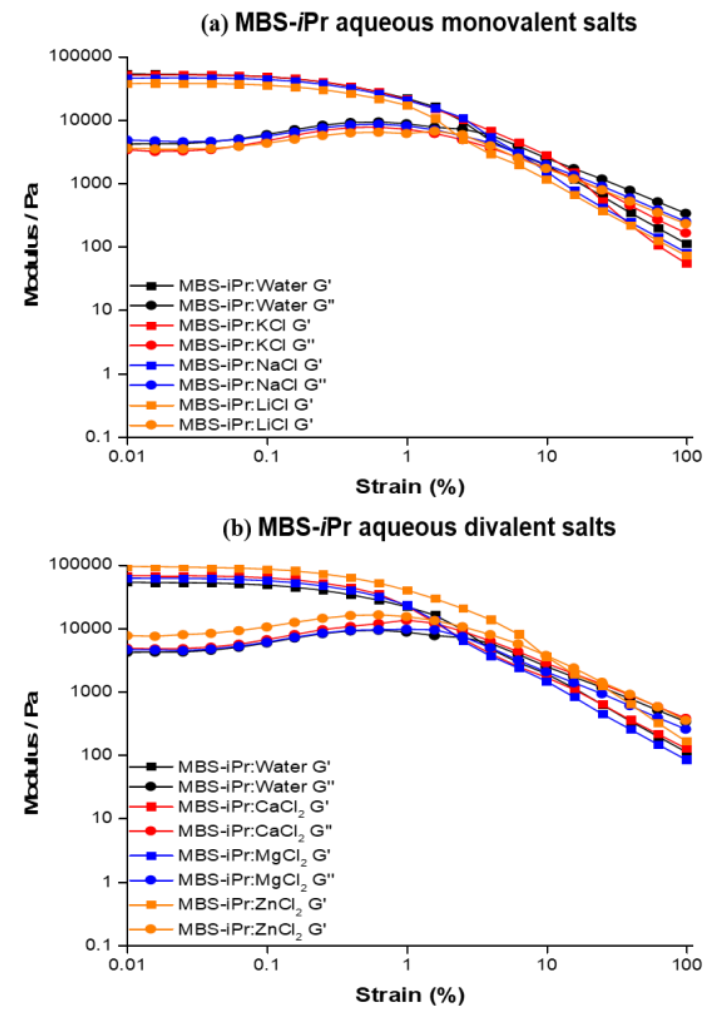

Fig. 13 | Amplitude sweep rhological data for MBS-iPr gels formed from water and $2 \% \mathrm{~W} / \mathrm{v}$ aqueous salt solutions at $1.0 \% \mathrm{w} / \mathrm{v}$ upon heating and cooling. $\mathrm{G}^{\prime}$ as Storage Modulus and $\mathrm{G}^{\prime \prime}$ as Loss Modulus.

Rheological data of MBS-iPr gels formed in water and $2 \% \mathrm{w} / \mathrm{v}$ monovalent and divalent salts solutions are summarised in Fig. 13. MBS-iPr gels formed from water and monovalent salts solutions (Fig. 13a) show a good G', G" and strain percentage. However, they do not exhibit strong and elastic gel features as high as the gels formed from divalent salt solutions. MBS-iPr gels formed from divalent aqueous salt solutions ( $2 \% \mathrm{w} / \mathrm{v})$ exhibit the highest value of $\mathrm{G}^{\prime}$ and $\mathrm{G}^{\prime \prime}$ with a strain value of $\sim 10 \%$. This parameter demonstrates appreciable elasticity of the gels and the positive effect that these divalent salts have on this property. 
On the other hand, Fig. 14 shows the rheological data of MBS-iPr and equimolar (DBSMBS)-iPr gels formed from ethanol-water solutions. The MBS-iPr gels display two trends where the higher the volume of water in the solution ratio, (i) the higher the value of $G^{\prime}$ and $\mathrm{G}^{\prime \prime}$, and (ii) the less elastic the gel is. Remarkably, the multicomponent gels formed from ethanol-water mixtures display a very similar trend to MBS-iPr. The higher the volume of water in the solution ratio, the higher the value of $\mathrm{G}^{\prime}$ and $\mathrm{G}^{\prime \prime}$. Conversely, it is apparent that the equimolar gel in 50:50 ethanol-water ratio exhibits the highest strain value and therefore it is the most elastic gel. This property can be ascribed to the morphology of the equimolar gel having a uniform-sized fibres in a weaved-like network (Fig. 10b).

The rapid formation of the gels during cooling makes measurement of the gel formation time difficult at ambient temperature in the thermal equilibrium state of the DBS-iPr gels. Also, transferring the sample is impractical as we could not obtain a reliable oscillatory measurement. This phenomenon means we cannot directly compare the gel strength of the monobenzylidene and dibenzylidene sorbitol gelators.

(a) MBS-iPr in ethanol/water solutions
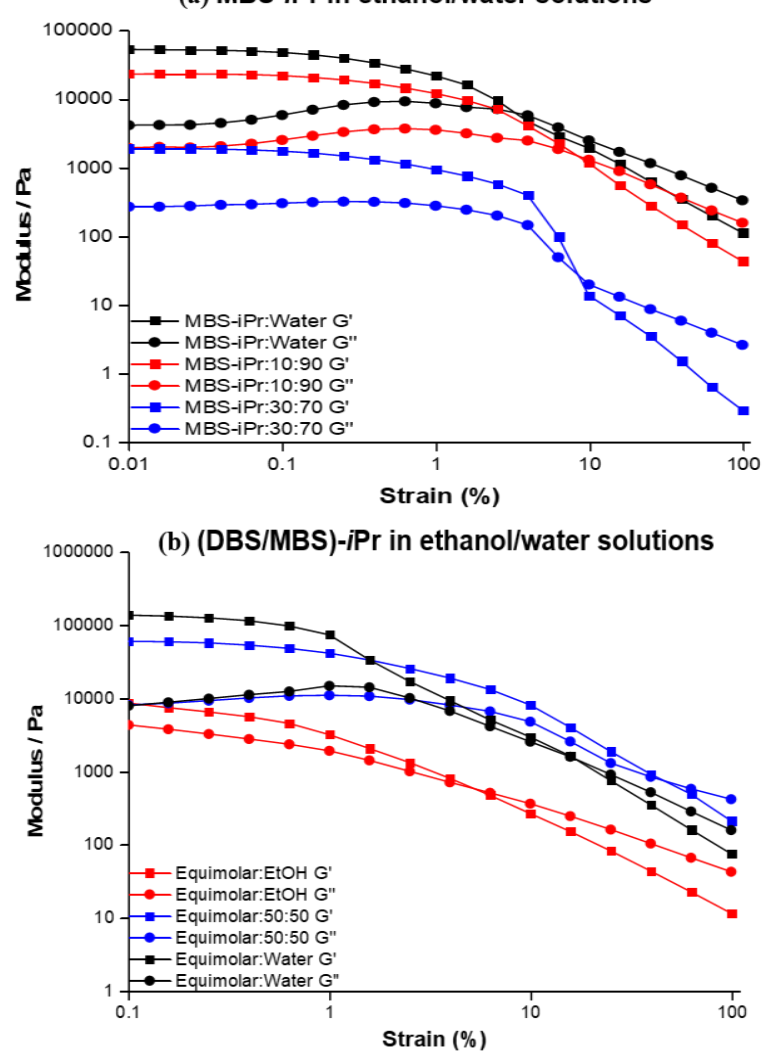

Fig. 14 | Amplitude sweep rhological data for: (a) MBS-iPr and (b) equimolar (DBS-MBS)-iPr gels formed from ethanol-water solutions at $1.0 \% \mathrm{~W} / \mathrm{v}$ upon heating and cooling. G' as Storage Modulus and G" as Loss Modulus.

\section{Xerogel Powder X-Ray Diffraction}

To explore and provide a direct comparison of the possible packing mode of the equimolar gelator samples, powder X-ray diffraction (PXRD) measurements were employed for the (i) MBS-iPr, (ii) equimolar MBS-iPr and DBS-iPr, and (iii) DBS-iPr xerogels, all from 20:80 ethanol-water solvent mixture (Fig. 15). The xerogels exhibited well-resolved X-ray 
diffraction patterns that were characteristic of a relatively long-range ordering of the molecules. The xerogels from the pure MBS-iPr and DBS-iPr gelators exhibit unique diffraction peaks where some of these peaks have relatively similar distances (Fig. 15).

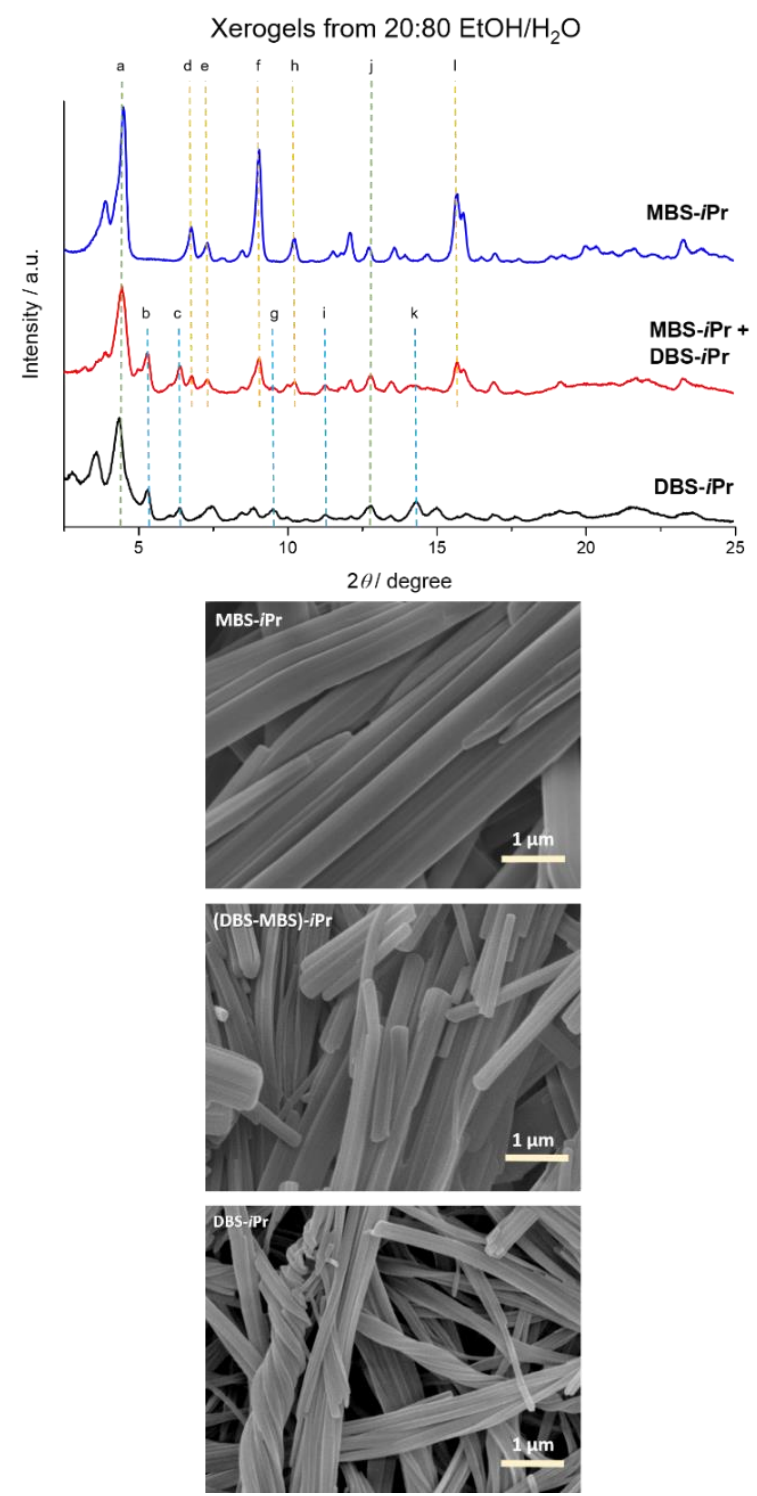

Fig. 15 | Powder X-ray diffraction patterns on xerogels of MBS-iPr, (DBS-MBS)-iPr, and DBS-iPr formed in 20:80 ethanol-water solution (top) and SEM images of these samples (bottom). Scale bar in images are all in $1 \mu \mathrm{m}$.

The diffraction pattern of the MBS-iPr xerogel displayed a series of sharp diffraction peaks with the main ones centred at $2 \theta=4.50^{\circ}(d=19.6 \AA), 9.06^{\circ}(d=9.75 \AA)$ and $15.7^{\circ}(d$ $=5.63 \AA)$. The $d$-spacing ratio is $1: 1 / 2: 1 / 3$, indicating that MBS-iPr assembles into a lamellar organisation ${ }^{59}$ with an interlayer distance of $19.6 \AA$ (evidenced by peak a in Fig. 15). ${ }^{25}$ As DBS-iPr has a sharp diffraction peak at $2 \theta=4.36^{\circ}$ which is similar to the MBS$i \operatorname{Pr} 19.6 \AA$ interlayer distance, we hypothesise that the interlayer distance of DBS-iPr is $20.2 \AA$. The larger spacing is expected because of the presence of an additional benzyl group when compared with MBS-iPr. There are no significant diffraction peaks that might indicate a strong contribution from $\Pi-\Pi$ stacking (in the region of $25^{\circ}$ ) but rather a general layered structure of the type shown in Fig. 4 is present.

The MBS-iPr diffractogram was plotted with the simulated PXRD data of MBS-Cinn and MBS-Van from their single crystal diffraction (ESI Fig.35). There are significant differences 
between the patterns of MBS-iPr and the crystalline materials, indicating a somewhat different organisation in the gel, and perhaps explaining why MBS-Van and MBS-Cinn do not gel.
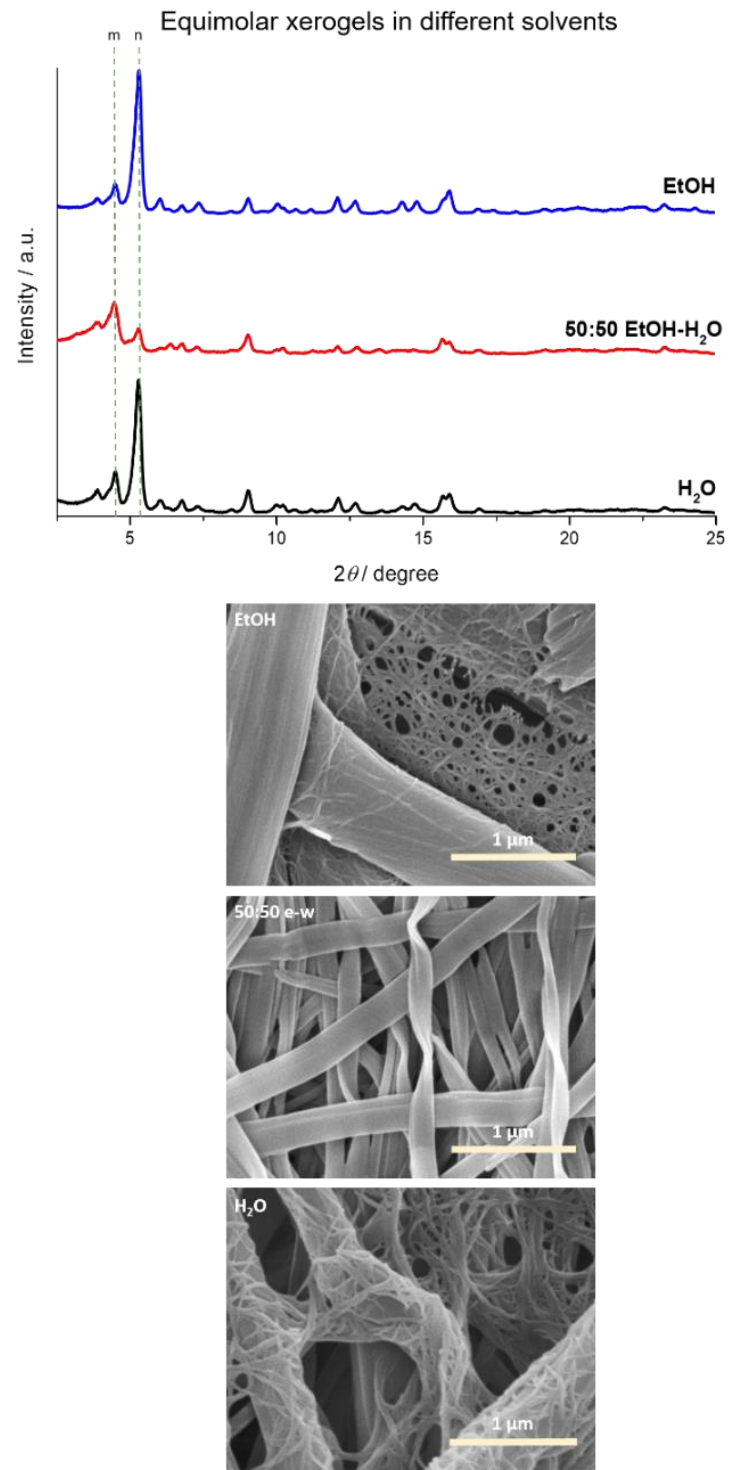

Fig. 16 | Powder X-ray diffraction patterns of equimolar xerogels in ethanol, 50:50 ethanol-water and water (top) and SEM images of these samples (bottom). Scale bar in images are all in $1 \mu \mathrm{m}$.

The equimolar mixture of gelators show distinctive DBS-iPr peaks at $2 \theta=5.32^{\circ}(d=16.6$ $\AA$, line $b), 6.32^{\circ}(d=13.8 \AA$, line $c), 9.52^{\circ}(d=9.28 \AA$, line $g), 11.3^{\circ}(d=7.84 \AA$, line i), $14.3^{\circ}(d=6.17 \AA$, line $k)$ and MBS-iPr peaks at $2 \theta=6.78^{\circ}(d=13.0 \AA$, line $d), 7.32^{\circ}$ $(d=12.1 \AA$, line $\mathrm{e}), 9.06^{\circ}(d=9.75 \AA$, line $\mathrm{f}), 10.3^{\circ}(d=8.61 \AA$, line $\mathrm{h}), 15.7^{\circ}(d=5.63$ $\AA$, line I) are seen present in the diffractogram. The observed peaks suggest that DBS-iPr and MBS-iPr self-assemble into layered structures independently in the equimolar gel. Some of the diffraction peaks occur at the same position for the pure xerogels and essentially coincide in the equimolar mixture (line $a$ and $j$ ). In contrast, interlayer diffraction peaks that are detected in MBS-iPr and DBS-iPr spectra between $3^{\circ}-4^{\circ}(\mathrm{d}=$ $22.6 \AA$ and $24.5 \AA$, respectively) are not present in the equimolar gel. This effect could be an indication of possible layering of unlike lamellae (for example, a DBS-iPr lamellar stacking on top of MBS-iPr fibre) in the 3D network leading to a lesser degree of long range crystalline order compared with the pure gelators. Therefore, self-sorting of the gelators 
would occur over a few lamellae, and, for the xerogels at least, lamellae of the other component form over the first-formed fibres. Co-assembly at the lamellar level is clearly no taking place because the diffraction data corresponding to short distances coincide over all samples for a given component.

Further PXRD experiments were performed for all the equimolar xerogels and are summarised in Fig. 16, showing (i) ethanol, (ii) 50:50 ethanol-water and (iii) water (all are plotted in ESI Fig. 34). The diffraction peaks arise at similar positions, however, a difference of intensity for the peaks associated to DBS-iPr at $2 \theta=5.32^{\circ}(d=16.6 \AA)$ and interlayer at $2 \theta=4.50^{\circ}(d=19.6 \AA)$ is apparent. From the solution of ethanol to $60: 40$ ethanol-water solution, the DBS-iPr peak is more intense than the interlayer peak. Interestingly, the opposite can be seen from 50:50 to 20:80 ethanol-water solution whereas the xerogel at 10:90 ethanol-water solution displays similar intensities. As it reaches pure water, the DBS-iPr diffraction peak, once again, had a stronger intensity. These observations could be related to the solubility of both DBS-iPr and MBS-iPr. Changing the solubility of the gelator in a solvent medium is the main factor in determining the outcome of gelation tests, as the gelator comes out of solution, it will self-assemble and form network fibres which immobilises the solvent.

We conclude that, with a higher ethanol content, DBS-iPr has more crystalline domains because self-assembly takes place readily in ethanol, whilst MBS-iPr stays as a solution in ethanol (Table 1). On the other hand, for lower ethanol content, DBS-iPr becomes less soluble whilst MBS-iPr self-assembles readily because of the higher water content. Therefore, DBS-iPr has less crystalline domains in this xerogel. As seen in the phase diagram in Fig. 5, from 10:90 ethanol-water solution to pure water, not all solid dissolved. The undissolved solid is believed to be DBS-iPr because of the very poor solubility in water and may cause the intensity of the DBS-iPr diffraction peak to increase in the diffractogram.

\section{Conclusion}

Two sustainable sorbitol derived gelators, DBS-iPr and MBS-iPr, can be prepared in good yields in a single step from green starting materials. DBS-iPr showed gelation properties in organic solvents and in ethanol-water mixtures. MBS-iPr on the other hand, can be classified as a hydrogelator that formed gels in water, including salt solutions with various dissolved ions such as $\mathrm{Na}^{+}, \mathrm{K}^{+}, \mathrm{Li}^{+}, \mathrm{Ca}^{2+}, \mathrm{Zn}^{2+}, \mathrm{Mg}^{2+}, \mathrm{Cl}^{-}$, and $\mathrm{SO}_{4}{ }^{-}$. SEM micrographs showed that DBS-iPr in a non-polar solvent, cyclohexane, resulted in helical fibres. IR results indicated that ethanol modified the intramolecular hydrogen bonding between the $-\mathrm{OH}$ groups.

The difference in solubility of the two gelators MBS-iPr and DBS-iPr were apparent hence we investigated the gelation of the two together as an equimolar multicomponent gel, (DBS-MBS)-iPr. This mixture displayed gelation in all ethanol-water mixtures and at a lower concentration than the single component system, which indicates that MBS-iPr has an influence on the gelation of DBS-iPr and vice-versa. However, co-assembly at the lamellar level does not take place, as indicated by both IR and PXRD results. Rather, the gelators self-sort and possibly layer through interactions of the hydroxyl groups, as indicated in the structural model in Figure 4.

Oscillatory rheology measurements showed that MBS-iPr and the equimolar DBS-MBS-iPr gel self-assembled to a much stronger gel in comparison to the DBS-iPr. A possible 
explanation for this effect is seen in the SEM images, where connections between the lamellae through hydrogen bonding of the sugar hydroxyl groups, already indicated in the IR spectroscopy and supported by the layering of the different gelators shown by the PXRD measurements.

The less bulky substituent on the mono-acetal compounds MBS-Van and MBS-Cinn, that did not exhibit any gelation properties from the solvents tested, may allow close packing that provides the crystals. On the other hand, these mono-acetal compounds were characterised with X-ray crystallography. The single crystal X-ray structures shows that the packing of both molecules are similar and are focusing on the hydrogen bonding between the sugar backbones. These structures provide the first precise supramolecular bonding motifs to this family of compounds. Indeed, IR spectroscopy indicates that hydrogen bonding is similar in the xerogels and crystals, and PXRD show a lamellar structure comprising sugar-aromatic reside alternating layers.

The gelation tests of the compounds has shown that solubility is a major factor in gelation properties, as in other systems. ${ }^{60}$ It is likely that in the multicomponent systems that solubility and effects of the two gelators upon one another's behaviour in solution have a determining effect on the outcome of the assembly, whereby these gels are stronger than the single component gels. Solubility is a factor in a related DBS gelator in a multicomponent system with a peptide amphiphile. ${ }^{61}$

The use of multicomponent gelling systems in a controlled way could lead to some interesting applications in soft materials for personal care products, polymer nucleation/clarification, and energy technology, some of which have already been accessed by this family of compounds. ${ }^{31,32,61}$

\section{Conflicts of interest}

"There are no conflicts to declare".

\section{Acknowledgements}

The Engineering and Physical Sciences Research Council (EPSRC) supported this work under grants (EP/N024818/1) and (EP/M00S178/1). The authors thank Dr Ajith Ravi Mallia for his help in single crystal data processing, Dr Hannah Constantin for her assistance with the PXRD and the Nanoscale and Microscale Research Centre (nmRC) in Nottingham. We also thank the anonymous referees for providing prompts for significant improvements to this article.

\section{Notes and references}

1 B. Gioia, N. Ben Ghalia and P. Kirilov, J. Pharm. Pharm. Sci., 2018, 7, 1-11.

2 P. K. Vemula and G. John, Acc. Chem. Res., 2008, 41, 769-782.

3 J. R. Silverman, M. Samateh and G. John, Eur. J. Lipid Sci. Technol., 2016, 118, 47-55.

4 S. Mukherjee, C. Shang, X. Chen, X. Chang, K. Liu, C. Yu and Y. Fang, Chem. Commun., 2014, 50, 13940-13943. 
J. H. Clark, V. Budarin, F. E. I. Deswarte, J. J. E. Hardy, F. M. Kerton, A. J. Hunt, R. Luque, D. J. Macquarrie, K. Milkowski, A. Rodriguez, O. Samuel, S. J. Tavener, R. J. White and A. J. Wilson, Green Chem., 2006, 8, 853-860.

S. Thakur, B. Sharma, A. Verma, J. Chaudhary, S. Tamulevicius and V. K. Thakur, J. Clean. Prod., 2018, 198, 143-159.

K. Prasad, Y. Kaneko and J. I. Kadokawa, Macromol. Biosci., 2009, 9, 376-382.

8

K. Yabuuchi, E. Marfo-Owusu and T. Kato, Org. Biomol. Chem., 2003, 1, 3464-3469.

9

N. Basu, A. Chakraborty and R. Ghosh, Gels, 2018, 4, 52.

B. O. Okesola and D. K. Smith, Chem. Soc. Rev., 2016, 45, 4226-4251.

B. O. Okesola and D. K. Smith, Chem. Commun., 2013, 49, 11164-11166.

H. Svobodová, Nonappa, M. Lahtinen, Z. Wimmer and E. Kolehmainen, Soft Matter, 2012, 8, 78407847.

K. Basu, N. Nandi, B. Mondal, A. Dehsorkhi, I. W. Hamley and A. Banerjee, Interface Focus, 2017, 7, 1-9.

E. Faggi, R. M. Sebastian and A. Valiribera, Tetrahedron, 2010, 66, 5190-5195.

X. Cao, Y. Li, Y. Yu, S. Fu, A. Gao and X. Chang, Nanoscale, 2019, 11, 10911-10920.

X. Cao, Q. Ding, Y. Li, A. Gao and X Chang, J. Mater. Chem. C, 2019, 7, 133-142.

D. B. Amabilino, D. K. Smith and J. W. Steed, Chem. Soc. Rev., 2017, 46, 2404-2420.

A. Prathap and K. M. Sureshan, Langmuir, 2019, 35, 6005-6014.

B. O. Okesola, V. M. P. Vieira, D. J. Cornwell, N. K. Whitelaw and D. K. Smith, Soft Matter, 2015, 11, 4768-4787.

S. Yamasaki, Y. Ohashi, H. Tsutsumi and K. Tsujii, Bull. Chem. Soc. Jpn., 1995, 68, 146-151.

B. O. Okesola, V. M. P. Vieira, D. J. Cornwell, N. K. Whitelaw and D. K. Smith, Soft Matter, 2015, 11, 4768-4787.

J. Li, K. Fan, X. Guan, Y. Yu and J. Song, Langmuir, 2014, 30, 13422-13429.

J. Li, K. Fan, L. Niu, Y. Li and J. Song, J. Phys. Chem. B, 2013, 117, 5989-5995.

W. C. Lai and P. H. Huang, Soft Matter, 2017, 13, 3107-3115.

E. A. Wilder and J. M. Antonucci, Macromol. Symp., 2005, 227, 255-263.

D. Wang, X. Zhang, Y. Liu, L. Li, Z. Bo, J. Zhou and H. Huo, Org. Electron. physics, Mater. Appl., 2017, 46, 158-165.

D. Knani and D. Alperstein, J. Phys. Chem. A, 2017, 121, 1113-1120.

Y. Ohsedo, Gels, 2016, 2, 13.

E. A. Wilder and J. M. Antonucci, Macromol. Symp., 2005, 227, 255-263.

E. A. Wilder, K. S. Wilson, J. B. Quinn, D. Skrtic and J. M. Antonucci, Chem. Mater., 2005, 17, 29462952.

M. Watase, Y. Nakatani and H. Itagaki, J. Phys. Chem. B, 1999, 103, 2366-2373.

J.M. Gardlik and R.V. Burkes, US Pat. 5106999, 1992.

T. Kobayashi, K. Fujitani and K. Murai, Eur. Pat. EP 0051681A1, 1982. 
R. Feng, L. Chen, Z. Hou and J. Song, Trans. Tianjin Univ., 2007, 13, 35-41.

T. Kobayashi, S. Kitagawa, H. Yagi, K. Fujitani, S. Sakai and T. Hasimoto, Nippon Kagaku Kaishi, 1994, 713-718.

E. A. Wilder, R. J. Spontak and C. K. Hall, Mol. Phys., 2003, 101, 3017-3027.

J. E. Campbell, J. Yang and G. M. Day, J. Mater. Chem. C, 2017, 5, 7574-7584.

K. Fan, H. Kong, X. Wang, X. Yang and J. Song, RSC Adv., 2016, 6, 80934-80938.

E. R. Draper and D. J. Adams, Chem, 2017, 3, 390-410.

W. C. Lai and Y. C. Lee, RSC Adv., 2016, 6, 98042-98051.

G. Socrates, Infrared and Raman characteristic group frequencies: tables and charts, Wiley, Chichester, U.K., 2001.

U. Liddel and E. D. Becker, Spectrochim. Acta, 1957, 10, 70-84.

W. B. Person and J. H. Newton, J. Chem. Phys., 1974, 61, 1040-1049.

E. R. Draper and D. J. Adams, Chem. Soc. Rev., 2018, 47, 3395--3405.

A. G. Shtukenberg, X. Cui, J. Freudenthal, E. Gunn, E. Camp and B. Kahr, J. Am. Chem. Soc., 2012, 134, 6354-6364.

A. G. Shtukenberg, A. Gujral, E. Rosseeva, X. Cui and B. Kahr, CrystEngComm, 2015, 17, 8817-8824.

A. G. Shtukenberg, Y. O. Punin, A. Gujral and B. Kahr, Angew. Chemie - Int. Ed., 2014, 53, 672-699.

J. Li, K. Fan, L. Niu, Y. Li and J. Song, J. Phys. Chem. B, 2013, 117, 5989-5995.

55

G. Ionita, M. Micutz, C. Munteanu, P. Ionita, T. Staicu, M. V. Neacsu, A. M. Ariciu and V. Tecuceanu, Appl. Magn. Reson., 2015, 46, 1395-1407.

L. E. Mears, E. R. Draper, A. M. Castilla, H. Su, Zhuola, B. Dietrich, M. C. Nolan, G. N. Smith, J. Doutch, S. Rogers, R. Akhtar, H. Cui and D. J. Adams, Biomacromolecules, 2017, 18, 3531-3540. K. J. Skilling, F. Citossi, T. D. Bradshaw, M. Ashford, B. Kellam and M. Marlow, Soft Matter, 2014, 10, 237-256.

60 A. R. Hirst, I. A. Coates, T. R. Boucheteau, J. F. Miravet, B. Escuder, V. Castelletto, I. W. Hamley and D. K. Smith, J. Am. Chem. Soc., 2008, 130, 9113-9121.

61 B. O. Okesola, Y. Wu, B. Derkus, S. Gani, D. Wu, D. Knani, D. K. Smith, D. J. Adams and A. Mata, Chem. Mater., 2019, 31, 7883-7897. 


\section{SUPPORTING INFORMATION}

\section{Experimental}

Materials and methods

All starting materials and solvents were purchased from standard chemical suppliers: Acros (cuminaldehyde 98\%, 4-TSA monohydrate 97.5\%, D-sorbitol 97\%), Merck (cinnamaldehyde 98\%, vanillin $98 \%$ ), Sigma (vanillin acetate $98 \%$ ).

Melting points were recorded on a Stuart SMP20. Optical rotations were recorded using an Anton Paar MCP100 Polarimeter, at $25.0^{\circ} \mathrm{C}$, at a concentration of $10 \mathrm{mg} \mathrm{mL}^{-1}$, equipped with a $2.50 \mathrm{~mm}$ cell length and $[\alpha]^{25}$ D values are given in deg $\mathrm{cm}^{2} \mathrm{~g}^{-1}$. NMR spectra were recorded on a Bruker Ascend 400 . FTIR spectra were recorded on a Bruker Alpha Platinum ATR. Mass Spectra were obtained using Bruker Compass MicroTOF, using electron sprai ionisation (ESI). CHN Analysis were obtained using the CE400 Elemental Analyzer, Exeter Analytical, INC. $1.6 \mathrm{mg}$ of each sample was combusted at temperature $975{ }^{\circ} \mathrm{C}$. Powder X-Ray diffraction (XRD) patterns were obtained by the Bruker D8 Advance with Da Vinci. $5 \mathrm{~mL}$ of each sample was prepared and were dried under reduced pressure to obtain xerogels. The xerogels were placed on a silicon wafer zero background sample holders for data acquisition in 2Theta scale between $1-65^{\circ}$, with step size of $0.02^{\circ}$, a step time of 6 seconds per step, using parallel beam mode at $40 \mathrm{kV}$ and $40 \mathrm{~mA}$. X-ray diffraction (XRD) data were collected in a Rigaku Oxford

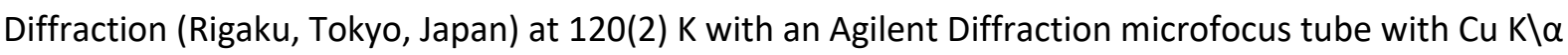
radiation type at 1.54184, equipped with an Atlas CCD area detector (S2). Scanning Electron Microscopy Measurements (SEM) samples were prepared by dropping a small amount of gel onto a SEM stub with a Pasteur pipette. The samples were left to dry in air overnight to give a xerogel, and then coated with iridium for imaging. For high resolution imaging on an FEG-SEM work, Iridium is the finest grading of coating and is recommended because it produce significantly better results than the other metal coatings. An argon plasma is used in a vacuum chamber to sputter particles of metal from the targets, which form a thin $(5 \mathrm{~nm})$ layer on the sample. Images of the xerogels were captured using a JEOL 7100F FEG-SEM microscope. Rheological measurements were taken using an Anton Paar Physica MCR 301 rheometer. Samples were heated to solution and were transferred into a mould on a rheometer plate. Samples were ensured to gel before rheological measurements were taken using a $50 \mathrm{~mm}$ cone plate. Gelation tests were performed using a Crystallisation Systems Crystal 16. Samples were heated from $20^{\circ} \mathrm{C}$ to $80^{\circ} \mathrm{C}$ at a rate of $5^{\circ} \mathrm{C} \mathrm{min}^{-1}$, held at $80^{\circ} \mathrm{C}$ for five minutes, and then cooled back to $20^{\circ} \mathrm{C}$ at a rate of $-5^{\circ} \mathrm{C} \mathrm{min}^{-1}$. Stirring was carried out on the ramp up at $800 \mathrm{rpm}$ using stirrer bars. No stirring was done during the hold or the ramp down to avoid disturbing any nascent fibres.

\section{Single crystal X-ray diffraction}

Single crystals were selected and mounted using Fomblin ${ }^{\circledR}$ (YR-1800 perfluoropolyether oil) on a polymer-tipped MiTeGen MicroMountTM and cooled rapidly to $120 \mathrm{~K}$ in a stream of cold $\mathrm{N}_{2}$ using an Oxford Cryosystems open flow cryostat. ${ }^{\text {ESI1 }}$ Single crystal X-ray diffraction data were collected on an Oxford Diffraction GV1000 (AtlasS2 CCD area detector, mirror-monochromated Cu-Ka radiation source; $\lambda=1.54184 \AA$, $\omega$ scans). Cell parameters were refined from the observed positions of all strong reflections and absorption corrections were applied using a Gaussian numerical method with beam profile correction (CrysAlisPro). ${ }^{\mathrm{ES} 12}$ Structures were solved within Olex2 ${ }^{\mathrm{ES} 13}$ by dual space iterative 
methods (SHELXT) ${ }^{\text {ESI4 }}$ and all non-hydrogen atoms refined by full-matrix least-squares on all unique F2 values with anisotropic displacement parameters (SHELXL). ESI5 Hydrogen atoms were refined with constrained geometries and riding thermal parameters. Structures were checked with checkCIF. ESI6 CCDC-1945762-1945763 contains the supplementary data for these compounds. These data can be obtained free of charge from The Cambridge Crystallographic Data Centre via www.ccdc.cam.ac.uk/data request/cif.

\section{Crystal structure refinement details}

MBS-Van The absolute configuration of the structure is determined by reference to the D-sorbitol starting material. Refinement of each configuration gives the same R1 value. Refinement of the opposite configuration gives a lower Flack parameter however this is not significant given the large uncertainty of the refined value.

The crystal was weakly diffracting with a resolution limit of $0.9 \AA$. The data was truncated to a resolution of $0.9 \AA$ resulting in a low data to parameter ratio, necessitating application of a large number of restraints to the cinnamyl moieties of the two residues (DFIX, DANG and FLAT). Rigid bond restraints were applied to the anisotropic displacement parameters of all atoms in the structure (RIGU).

The cinnamyl moiety of residue $B$ is disordered over two conformations the occupancies of which have been refined and constrained to sum to unity, having values of $0.65(4)$ and $0.35(4)$. The anisotropic displacement parameters of the disordered moieties have been restrained to be similar (SIMU). Geometric restraints applied to the 1,2 and 1,3 distances in the disordered moieties were calculated using Grade Web Server v1.104. The anisotropic displacement parameters of disordered atoms $\mathrm{C} 17 \mathrm{C} / \mathrm{B}$ and C18C/B have been restrained to have more isotropic character (ISOR).

Hydrogen atoms bound to carbon atoms in the structure were geometrically placed and refined using a riding model. Hydroxyl hydrogen atoms were not observed in the electron density map and are geometrically placed to donate hydrogen bonds to appropriate acceptors. Geometric placement of hydroxyl atoms on O4B and O12B clashed with hydrogen atoms of adjacent hydroxyl groups and were omitted from the model. Their correct positions could not be determined from the electron density map and it is likely that many of the hydrogen bonds are in fact disordered with roles of donors and acceptors interchangeable. The omitted hydrogen atoms are included in the unit cell contents.

MBS-Cinn Hydrogen atoms attached to carbon atoms were observed in the electron density map before being geometrically placed and refined using a riding model. The positions of hydroxylhydrogen atoms $\mathrm{H} 8, \mathrm{H} 8, \mathrm{H} 12$ and $\mathrm{H} 22$ are refined with their $\mathrm{O}-\mathrm{H}$ bond distances restrained to a target value of $0.84 \AA$ (DFIX). Hydroxy-hydrogen atom $\mathrm{H} 2$ was geometrically placed and refined with a riding model (AFIX 147). The isotropic displacement parameters of the hydroxyl-hydrogen atoms are fixed at a value of 1.5 time Ueq of their parent oxygen atoms.

\section{Synthetic procedures}

\section{General Methods}

D-sorbitol (1.0 eq.) and 4-toluene sulfonic acid (4-TSA) (0.2 eq.) were transferred into a roundbottomed flask and were stirred in $\mathrm{MeOH}(100 \mathrm{~mL})$ in room temperature. The aldehyde of choicecuminaldehyde, vanillin and cinnamaldehyde (1.0 eq. for the mono and 2.0 eq. for the di) was then added dropwise and the reaction was left stirring overnight. The reaction mixture was evaporated under reduced pressure to obtain white solid. The white solid was digested in $\mathrm{H}_{2} \mathrm{O}(100 \mathrm{~mL})$ and was 
filtered under reduced pressure. The filter was then washed with EtOAc $(50 \mathrm{~mL})$ followed by $\mathrm{Et}_{2} \mathrm{O}(50$ $\mathrm{mL}$ ) and dried in vacuo to yield the titled product as a white powder.

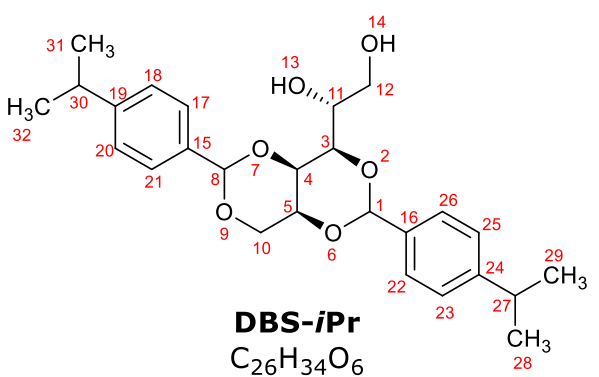

Synthesis of DBS-iPr: DBS-iPr: The titled compound was synthesized using the general procedure with cuminaldehyde. Precipitate formed and was filtered instead of evaporation under reduced pressure. Rest of the work-up followed but was furthered washed with cold $\mathrm{MeOH}$ (100 $\mathrm{mL}$ ) to remove all the MBS-iPr. Yield: (45\%). M.p.: 193-195 ${ }^{\circ} \mathrm{C} .{ }^{1} \mathrm{H}$ NMR $\left(500 \mathrm{MHz}, \mathrm{CD}_{3} \mathrm{CN}\right) \delta 7.44(4 \mathrm{H}, \mathrm{dd}, J=8.4,2.4,17-$ $\underline{H}, 21-\underline{H}, 26-\underline{H}, 22-\underline{H}), 7.30(4 \mathrm{H}, \mathrm{d}, J=8.0,18-\underline{H}, 20-\underline{H}, 25-\underline{H}$, 23- $\underline{H}), 5.67(1 \mathrm{H}, \mathrm{s}, 8-\underline{H}), 5.66(1 \mathrm{H}, \mathrm{s}, 1-\underline{H}), 4.21\left(2 \mathrm{H}, \mathrm{dd}, J=4.4,1.8,10-\underline{H}_{2}\right), 4.16(1 \mathrm{H}, \mathrm{t}, J=1.4,5-\underline{H})$, $3.96-3.81(3 \mathrm{H}, \mathrm{m}, 4-\underline{H}, 3-\underline{H}, 11-\underline{H}), 3.69\left(1 \mathrm{H}, \mathrm{ddd}, J=11.4,5.8,2.7,12-\underline{H}_{\mathrm{a}}\right), 3.59(1 \mathrm{H}, \mathrm{dt}, J=11.2,5.3$, $\left.12-\underline{H}_{\mathrm{b}}\right), 3.19(1 \mathrm{H}, \mathrm{d}, J=5.4,13-\underline{H}), 2.95(2 \mathrm{H}$, hept, $J=6.9,27-\underline{H}, 30-\underline{H}), 2.72(1 \mathrm{H}, \mathrm{t}, J=6.1,14-\underline{H}), 1.26$ $\left(12 \mathrm{H}, \mathrm{d}, J=6.9,28-\underline{H}_{3}, 29-\underline{H}_{3}, 30-\underline{H}_{3}, 31-\underline{H}_{3}\right) .{ }^{13} \mathrm{C} \mathrm{NMR}\left(101 \mathrm{MHz}, \mathrm{CD}_{3} \mathrm{CN}\right) \delta 149.7$ (C15), 149.6 (C16), 136.5 (C19), 136.3 (24), 126.2 (C17), 126.2 (C21), 126.1 (C26), 126.1 (C22), 100.1 (C8), 100.0 (C1), 77.9 (C3), 70.4 (C4), 69.7 (C10), 68.9 (C5), 68.2 (C11), 62.8 (C12), 33.7 (C27, C30), 23.3 (C31, C32, C29, C28). $\mathrm{v}_{\max } / \mathrm{cm}^{-1} 3260 \mathrm{br}\left(\mathrm{OH}\right.$ sugar), 2954w, 2871w, 1398w, 1339w, 1013s. (ESI) $\mathrm{m} / \mathrm{z}\left(\mathrm{M}+\mathrm{H}_{4} \mathrm{~N}\right)^{+}$calcd. for $\mathrm{C}_{26} \mathrm{H}_{38} \mathrm{NO}_{6}{ }^{+} 460.2694$, found 460.2693. [ $\left.\alpha\right]^{25}{ }_{\mathrm{D}}=+60.0$ (c. $10.0 \mathrm{mg} \mathrm{mL}^{-1}$, DMSO). CHN Analysis: Calcd (\%) C 70.55; H 7.75; O 21.70; Found (100\%) C 68.43, H 7.75, 0 23.95.

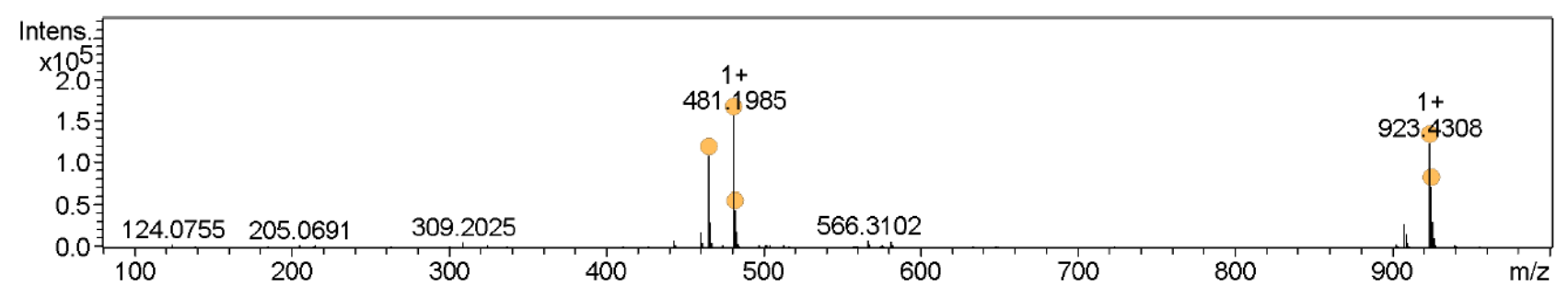

SI Fig. 1| HRMS of DBS-iPr in positive mode 


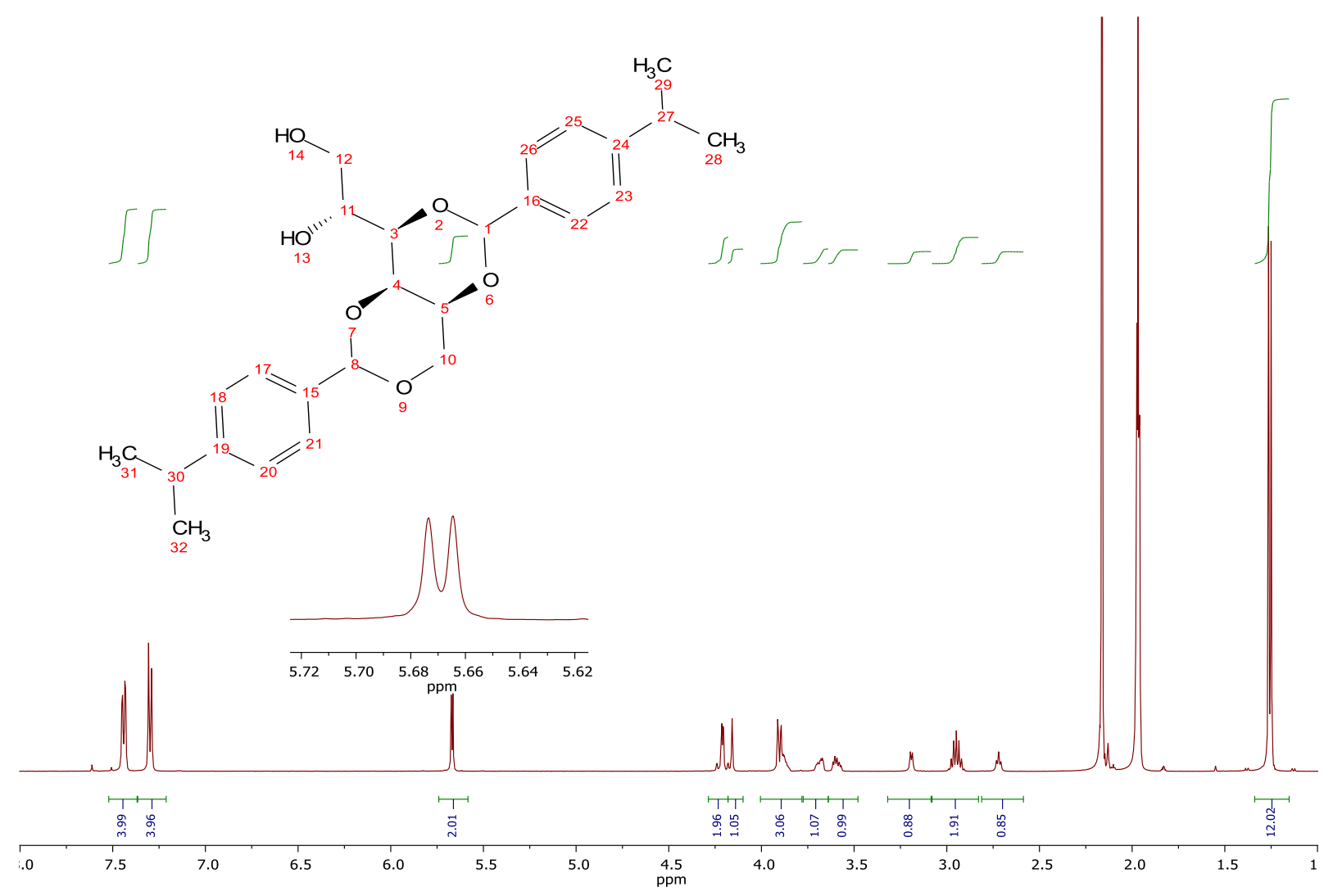

SI Fig. $2 \mid{ }^{1} \mathrm{H}$ NMR of DBS-iPr

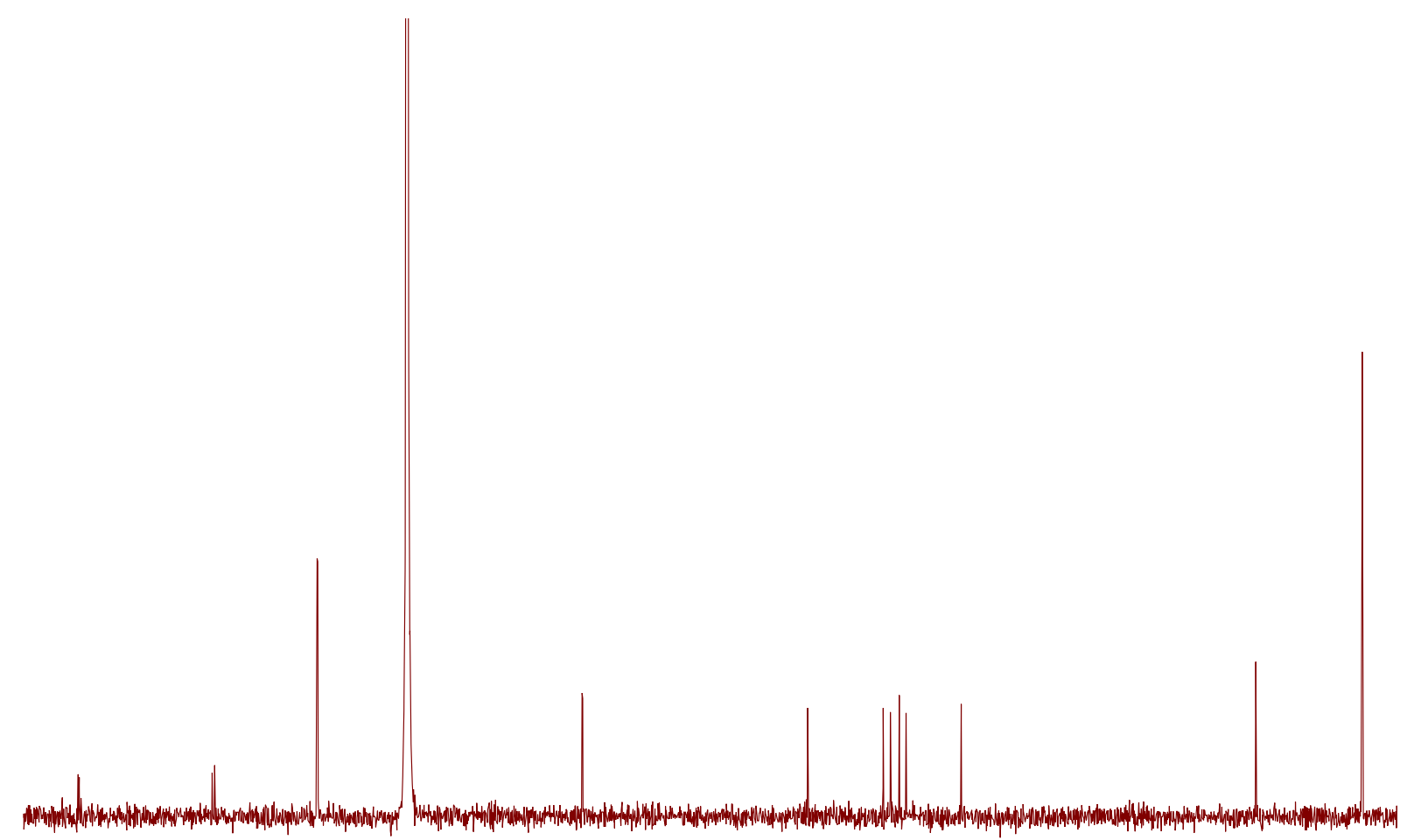

$\begin{array}{llllllllllllllllllllllllllllllllllllll}55 & 150 & 145 & 140 & 135 & 130 & 125 & 120 & 115 & 110 & 105 & 100 & 95 & 90 & 85 & 80 & 75 & 70 & 65 & 60 & 55 & 50 & 45 & 40 & 35 & 30 & 25 & 2\end{array}$

SI Fig. $3 \mid{ }^{13} \mathrm{C}$ NMR of DBS-iPr 


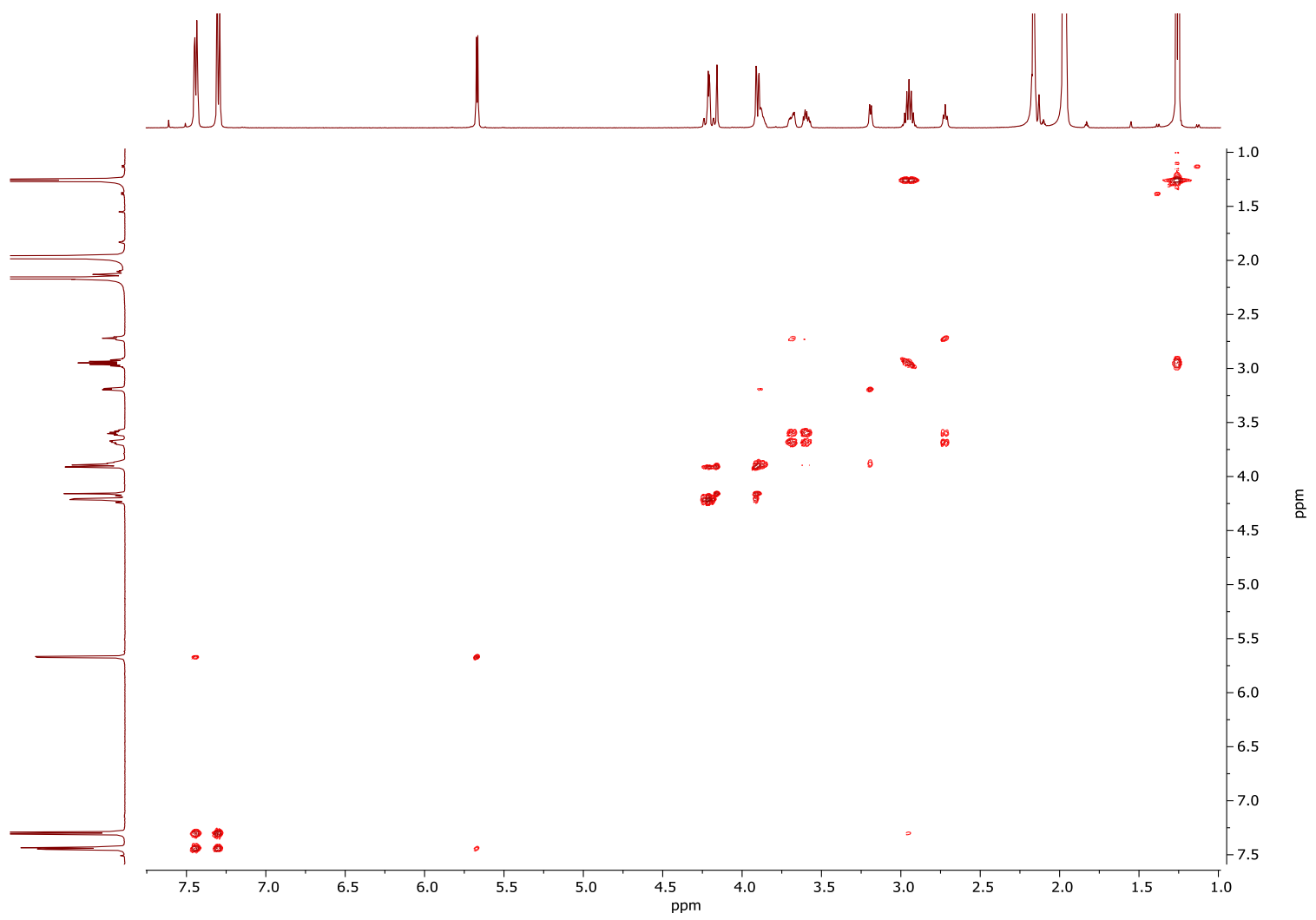

SI Fig. 4 | COSY of DBS-iPr

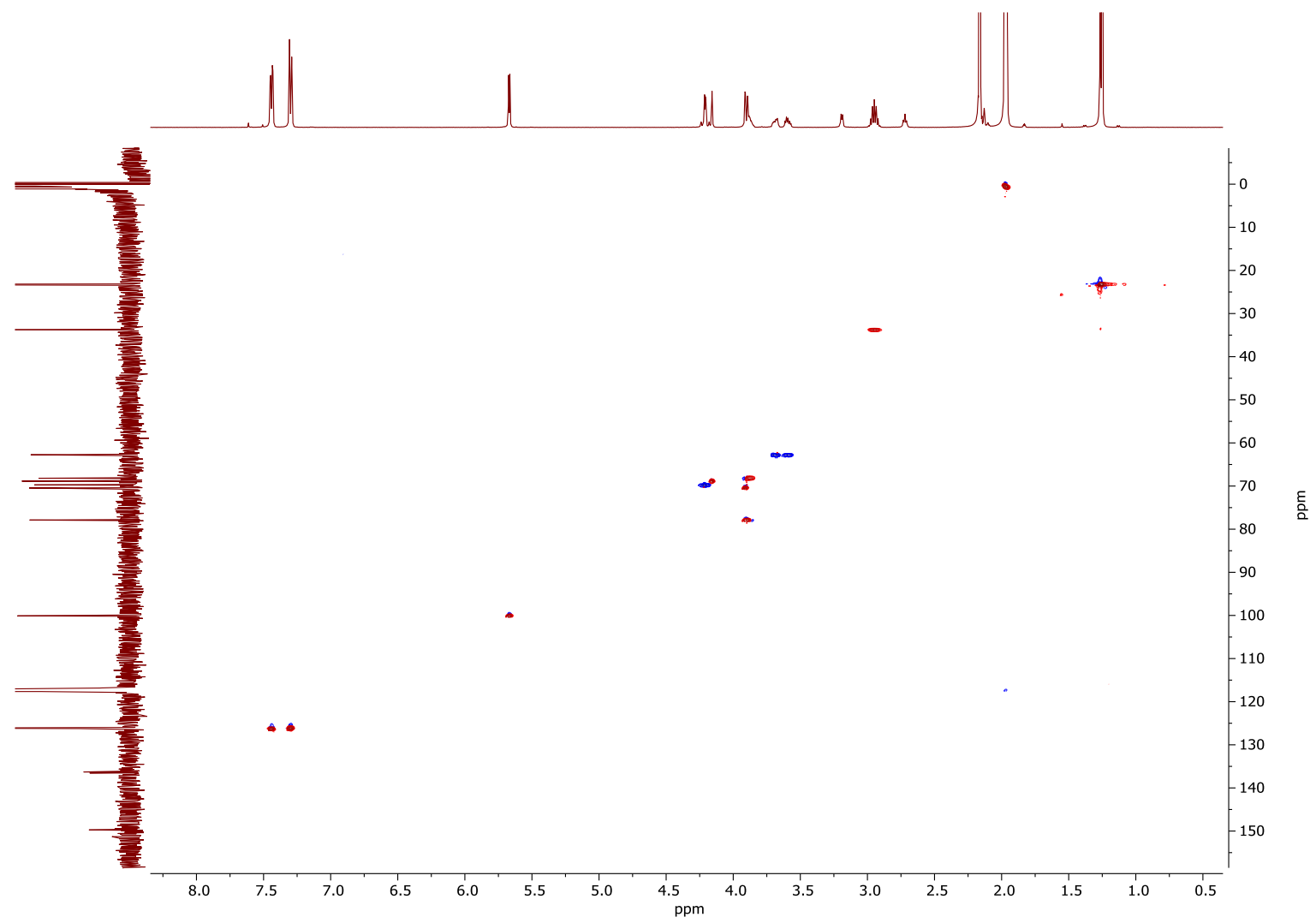

SI Fig. 5 | HSQC of DBS-iPr

32 


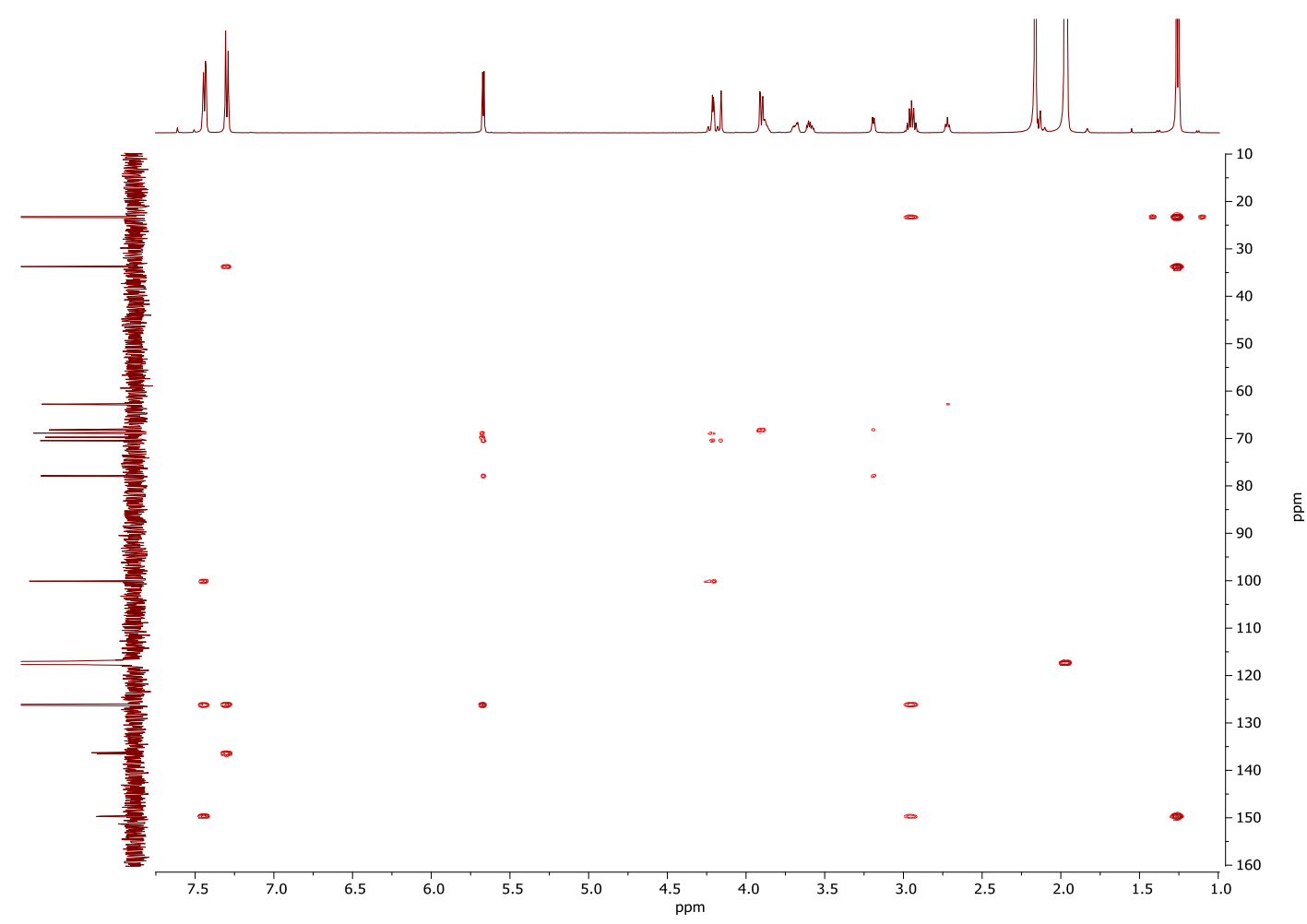

SI Fig. 6 | HMBC of DBS-iPr

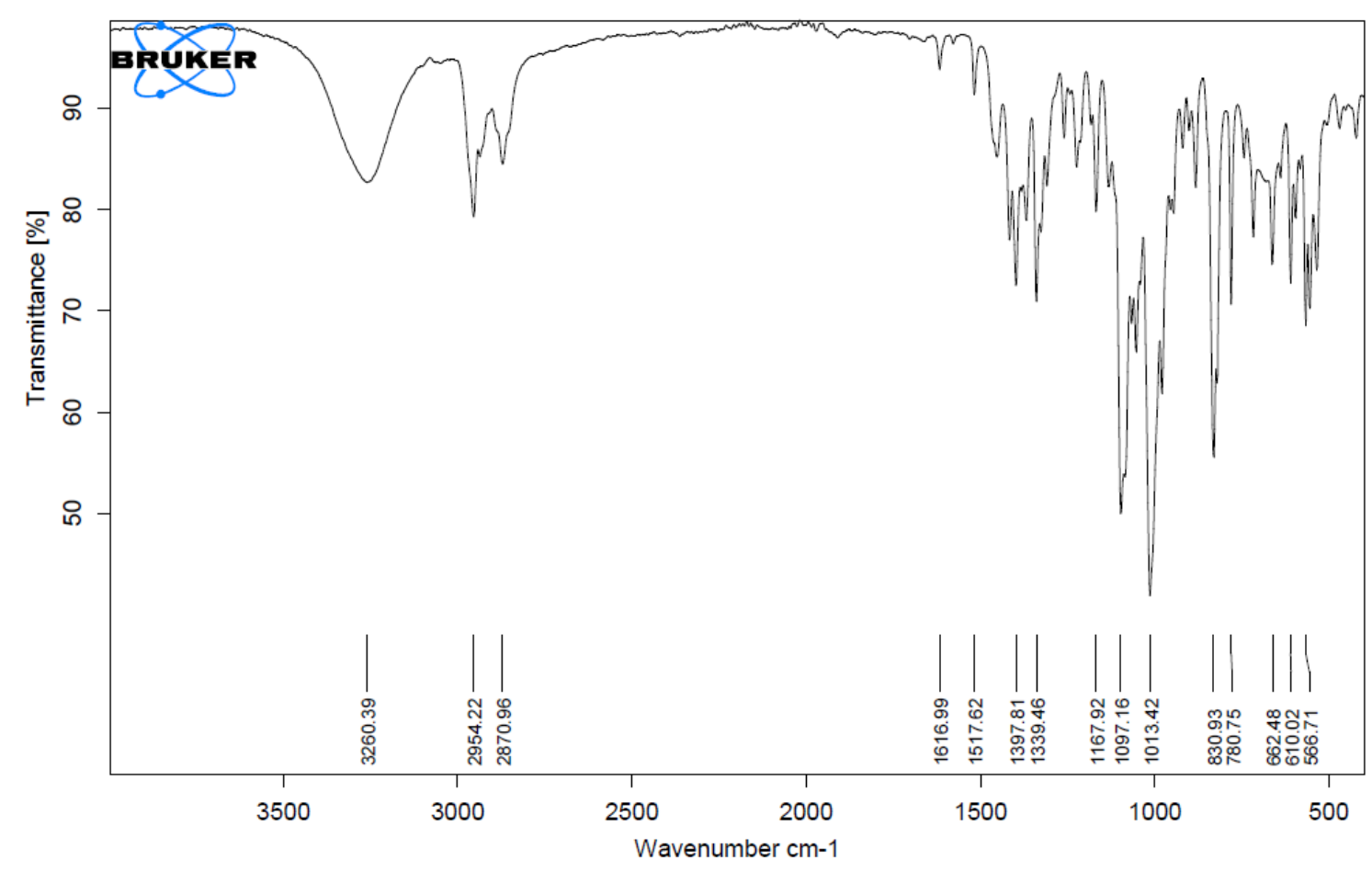

SI Fig. 7 | IR spectrum of DBS-iPr 


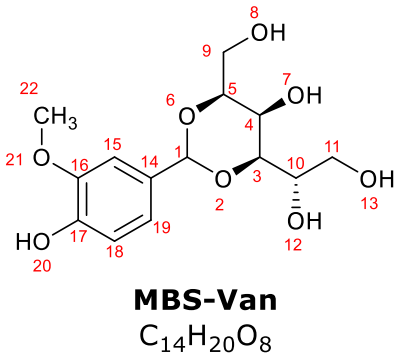

Synthesis of MBS-Van: The reaction was carried out in inert atmosphere following the general procedure. However, this compound was not washed with water because it is soluble in water. Yield (73\%). Mp $173-$ $175^{\circ} \mathrm{C} .{ }^{1} \mathrm{H}$ NMR (400 MHz, DMSO- $\left.d_{6}\right) \delta 8.99(1 \mathrm{H}, \mathrm{s}, 20-\underline{H}), 7.06(1 \mathrm{H}, \mathrm{s}, 15-$ $\underline{H}), 6.88(1 \mathrm{H}, \mathrm{d}, J=8.1,19-\underline{H}), 6.73(1 \mathrm{H}, \mathrm{d}, J=8.1,18-\underline{H}), 5.43(1 \mathrm{H}, \mathrm{s}, 1-\underline{H})$, $4.68(1 \mathrm{H}, \mathrm{d}, J=5.9,12-\underline{H}), 4.64(1 \mathrm{H}, \mathrm{t}, J=5.8,8-\underline{H}), 4.39(1 \mathrm{H}, \mathrm{t}, J=5.8,13-$ $\underline{H}), 4.33(1 \mathrm{H}, \mathrm{d}, J=8.2,7-\underline{H}), 3.77-3.73\left(4 \mathrm{H}, \mathrm{m}, 22-\underline{H}_{3}, 5-\underline{H}\right), 3.73-3.65$ $(2 \mathrm{H}, \mathrm{m}, 10-\underline{H}, 4-\underline{H}), 3.64-3.49\left(4 \mathrm{H}, \mathrm{m}, 3-\underline{H}, 9-\underline{H}_{2}, 11-\underline{H}_{\mathrm{b}}\right), 3.42-3.37(1 \mathrm{H}$, m, 11- $\underline{H}_{\mathrm{a}}$ ). ${ }^{13} \mathrm{C}$ NMR (100 MHz, DMSO- $d_{6}$ ) $\delta 147.5$ (C17), 147.2 (C14), 130.5 (C16), 119.8 (C19), 115.1 (C18), 111.3 (C15), 101.0 (C1), 81.4 (C5), 79.9 (C3), 69.6 (C10), 63.2 (C11), 62.1 (C4), 61.4 (C9), 56.2 (C22). $v_{\max } / \mathrm{cm}^{-1} 3461 \mathrm{w}(\mathrm{Ph}-\mathrm{OH}), 3262 \mathrm{br}$ (OH sugar), 2967w, 1618w, 1095s, 1016s. (ESI) $\mathrm{m} / \mathrm{z}(\mathrm{M}+\mathrm{Na})^{+}$ calcd. for $\mathrm{C}_{14} \mathrm{H}_{20} \mathrm{NaO}_{8}{ }^{+} 339.1050$, found 339.1043. $[\alpha]^{25}{ }_{\mathrm{D}}=+8.00$ (c. $10.0 \mathrm{mg} \mathrm{mL}^{-1}, \mathrm{H}_{2} \mathrm{O}$ ). CHN Analysis: Calcd (\%) C 54.86; H 6.14; O 39.00; Found (100\%) C 53.12, H 6.44, O 40.44.
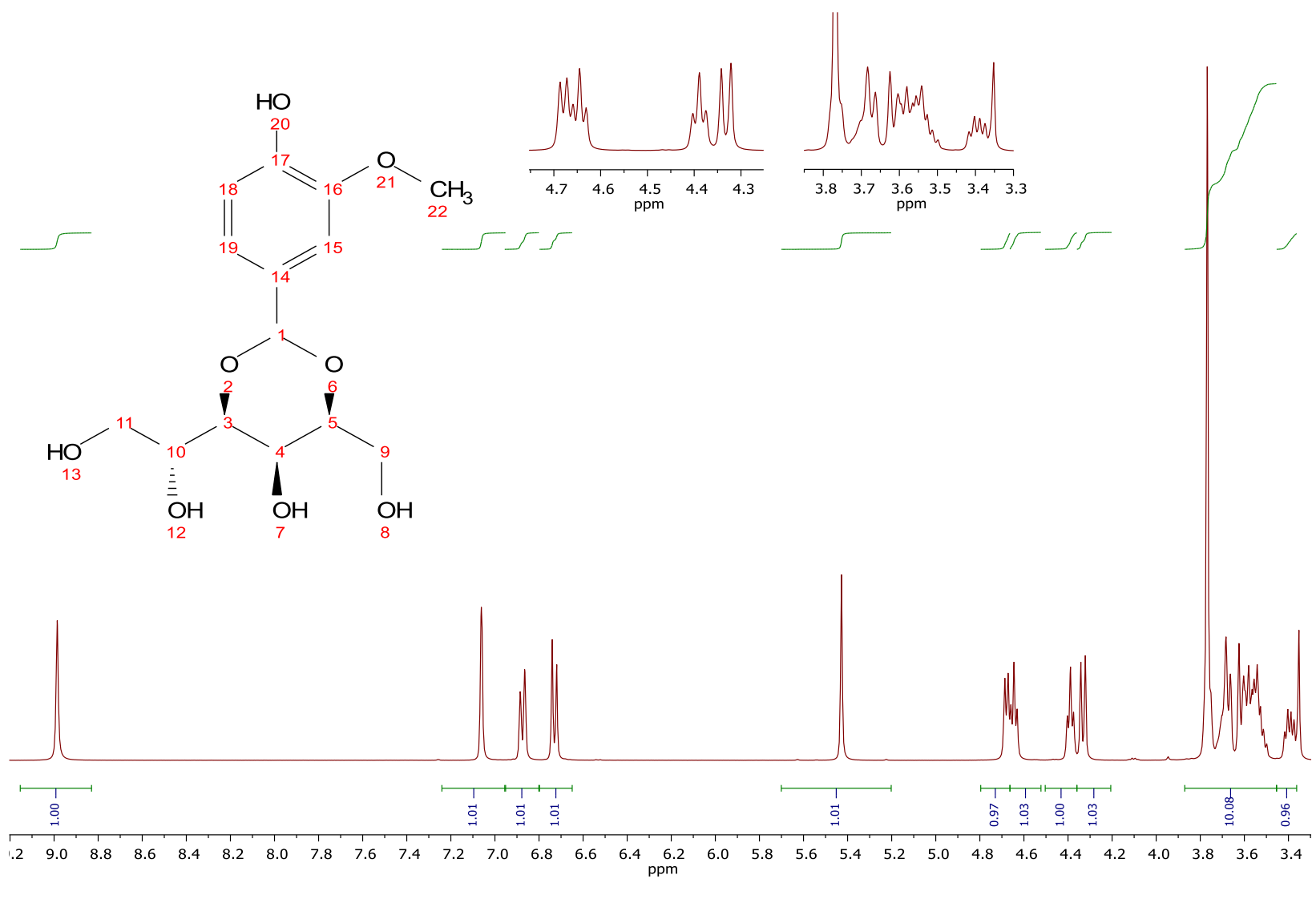

SI Fig. $8 \mid{ }^{1} \mathrm{H}$ NMR of MBS-Van 

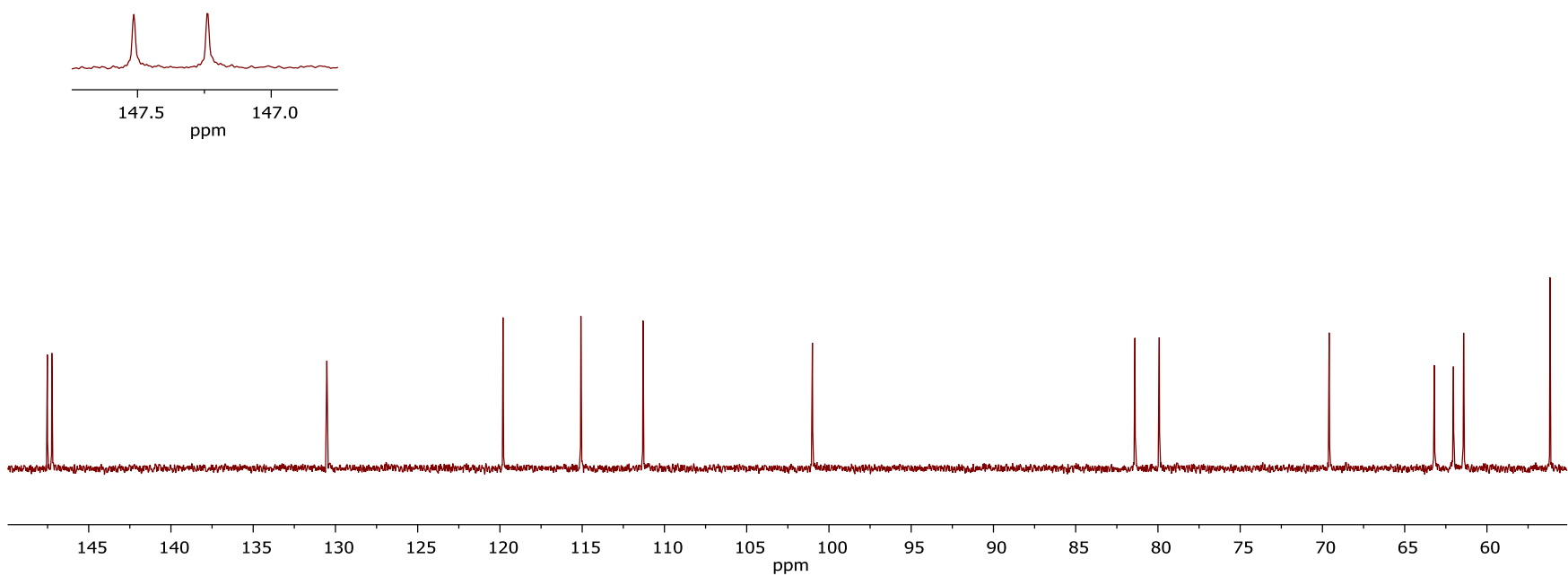

SI Fig. $9 \mid{ }^{13} \mathrm{C}$ NMR of MBS-Van

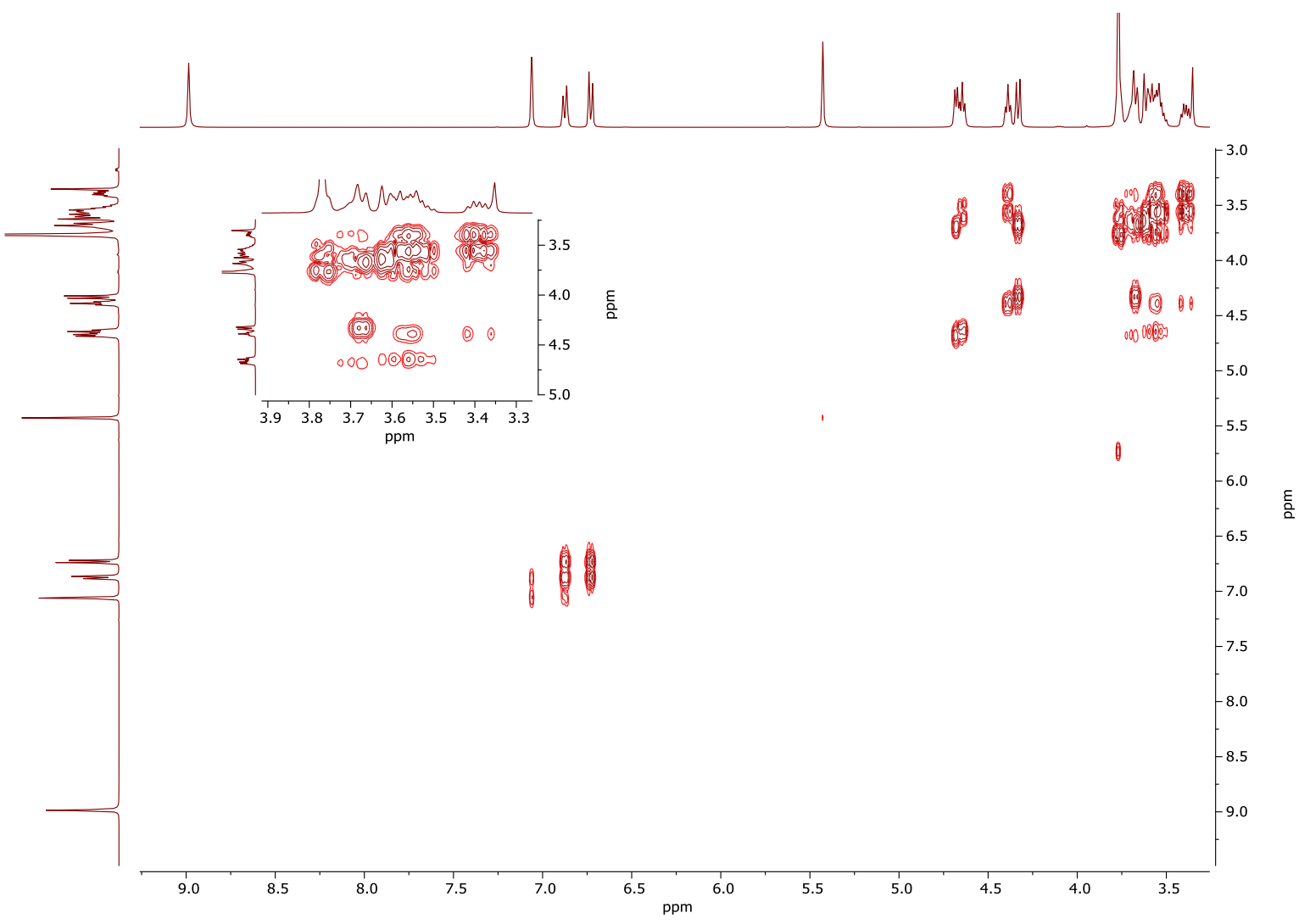

SI Fig. 10 | COSY of MBS-Van 


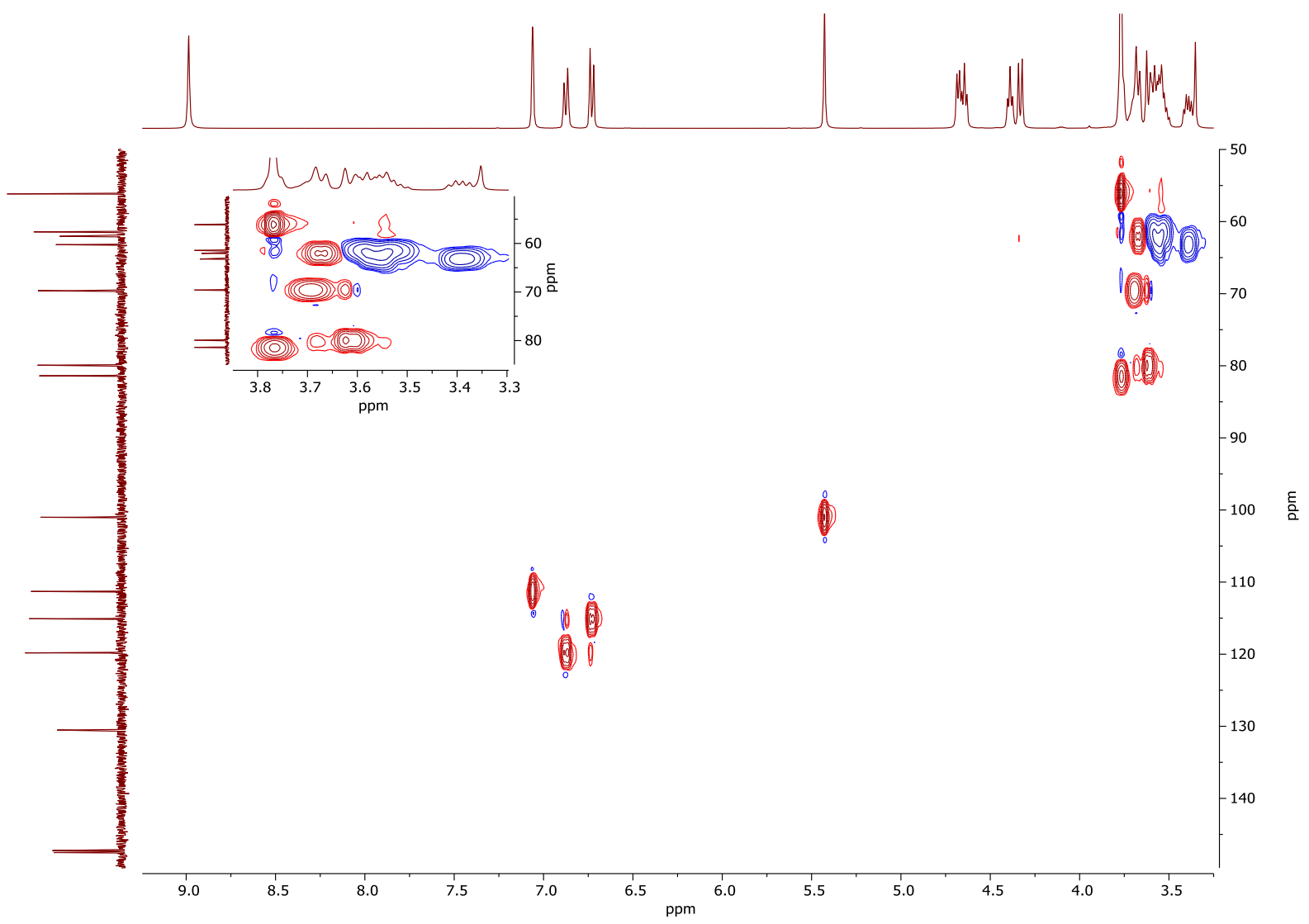

SI Fig. 11 |HSQC of MBS-Van

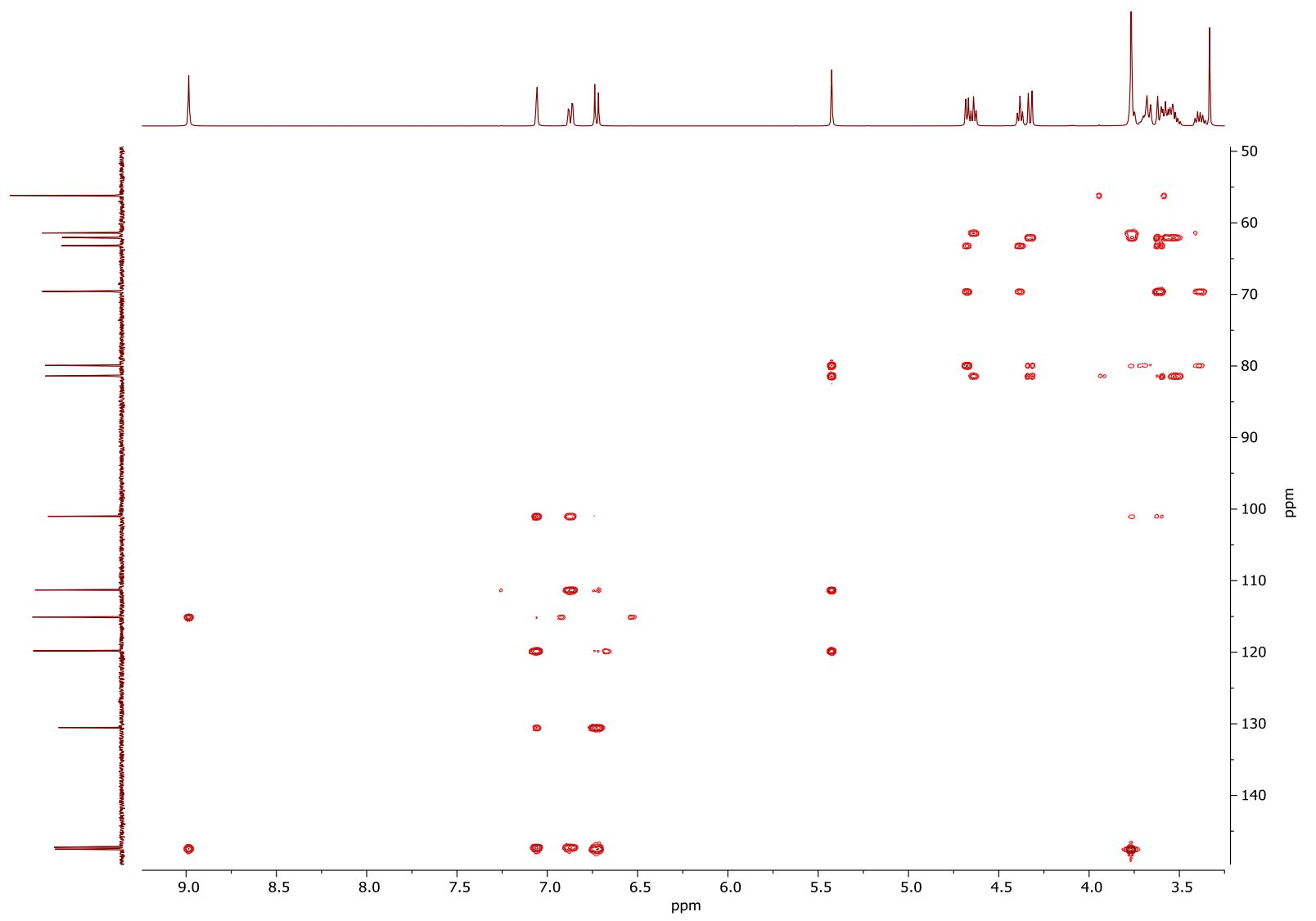

SI Fig. 12 |HMBC of MBS-Van 


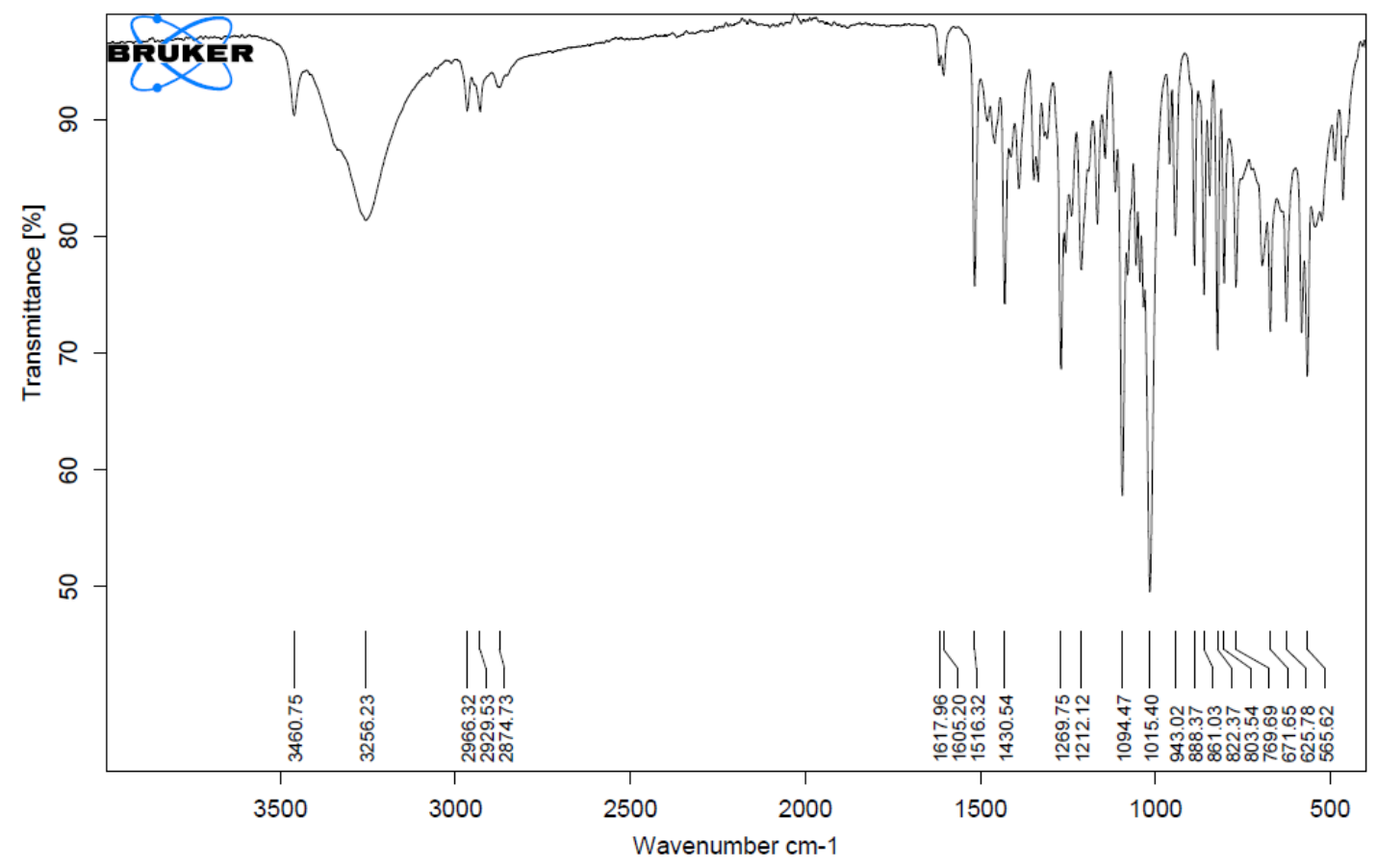

SI Fig. 13 | IR spectrum of MBS-Van
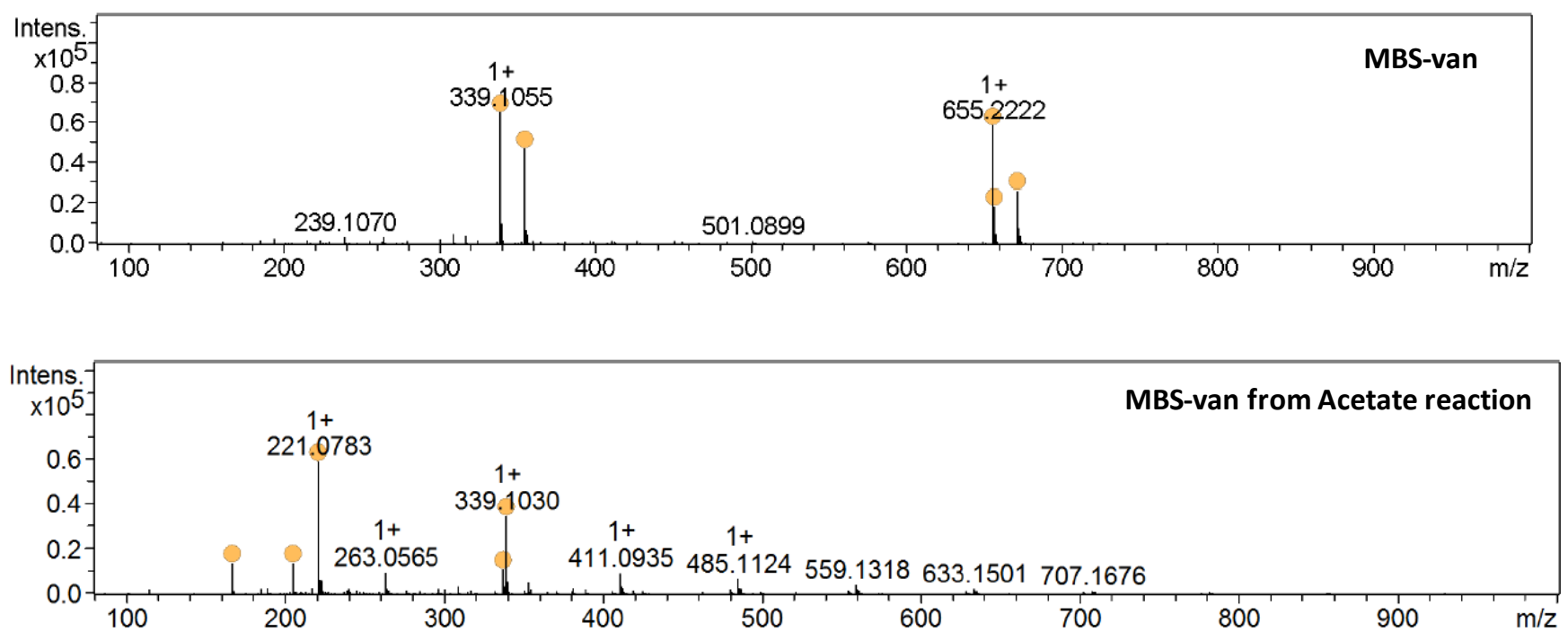

SI Fig. 14 | HRMS of MBS-Van 


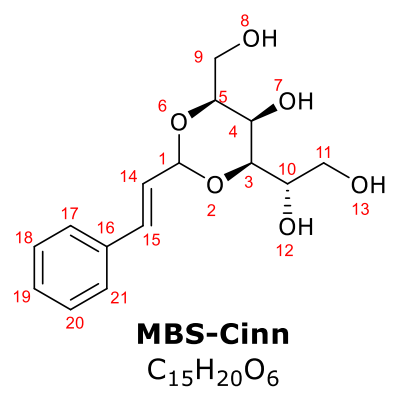

Synthesis of MBS-Cinn: The titled compound was synthesized via general procedure. Yield a white solid (68\%). Mp $124-126^{\circ} \mathrm{C} .{ }^{1} \mathrm{H}$ NMR $(400 \mathrm{MHz}$, DMSO- $\left.d_{6}\right) \delta 7.51-7.46(2 \mathrm{H}, \mathrm{m}, 17-\underline{H}, 21-\underline{H}), 7.40-7.33(2 \mathrm{H}, \mathrm{m}, 18-\underline{H}, 20-$ H), $7.32-7.26(1 \mathrm{H}, \mathrm{m}, 19-\underline{H}), 6.74(1 \mathrm{H}, \mathrm{d}, J=16.2,14-\underline{H}), 6.23(1 \mathrm{H}, \mathrm{dd}, J$ $=16.2,5.1,15-\underline{H}), 5.19(1 \mathrm{H}, \mathrm{dd}, J=5.1,1.1,1-\underline{H}), 4.69(1 \mathrm{H}, \mathrm{d}, J=6.0,12-$ $\underline{H}), 4.65(1 \mathrm{H}, \mathrm{t}, J=5.7,8-\underline{H}), 4.42(1 \mathrm{H}, \mathrm{t}, J=5.8,13-\underline{H}), 4.36(1 \mathrm{H}, \mathrm{d}, J=7.3$, 7- $\underline{H}), 3.74-3.64(3 \mathrm{H}, \mathrm{m}, 5-\underline{H}, 10-\underline{H}, 4-\underline{H}), 3.62-3.48\left(4 \mathrm{H}, \mathrm{m}, 3-\underline{H}, 9-\underline{H}_{2}\right.$, $\left.11-\underline{H}_{\mathrm{b}}\right), 3.42\left(1 \mathrm{H}, \mathrm{dt}, J=11.3,5.7,11-\underline{H}_{\mathrm{a}}\right) \mathrm{ppm} .{ }^{13} \mathrm{C} \mathrm{NMR}(100 \mathrm{MHz}$, DMSO-

$\left.d_{6}\right) \delta 136.2$ (C16), 133.0 (C14), 129.2 (C17, C21), 128.7 (C19), 127.1 (C18, C20), 126.8 (C15), 100.2 (C1), 81.0 (C5), 79.4 (C3), 69.7 (C10), 63.1 (C11), 62.0 (C4), 61.4 (C9) ppm. $v_{\max } / \mathrm{cm}^{-1} 3271 \mathrm{br}$ (OH sugar), 2933w, 2864w, 965s. (ESI) $\mathrm{m} / z$ (M+Na) ${ }^{+}$calcd. for $\mathrm{C}_{15} \mathrm{H}_{20} \mathrm{NaO}_{6}{ }^{+} 319.1152$, found 319.1144. $[\alpha]^{25}{ }_{\mathrm{D}}=+8.00$ (c. $10.0 \mathrm{mg} \mathrm{mL}^{-1}$, MeOH). CHN Analysis: Calcd (\%) C 60.78; H 6.81; O 32.41; Found (100\%) C 58.00, H 6.69, 035.31.
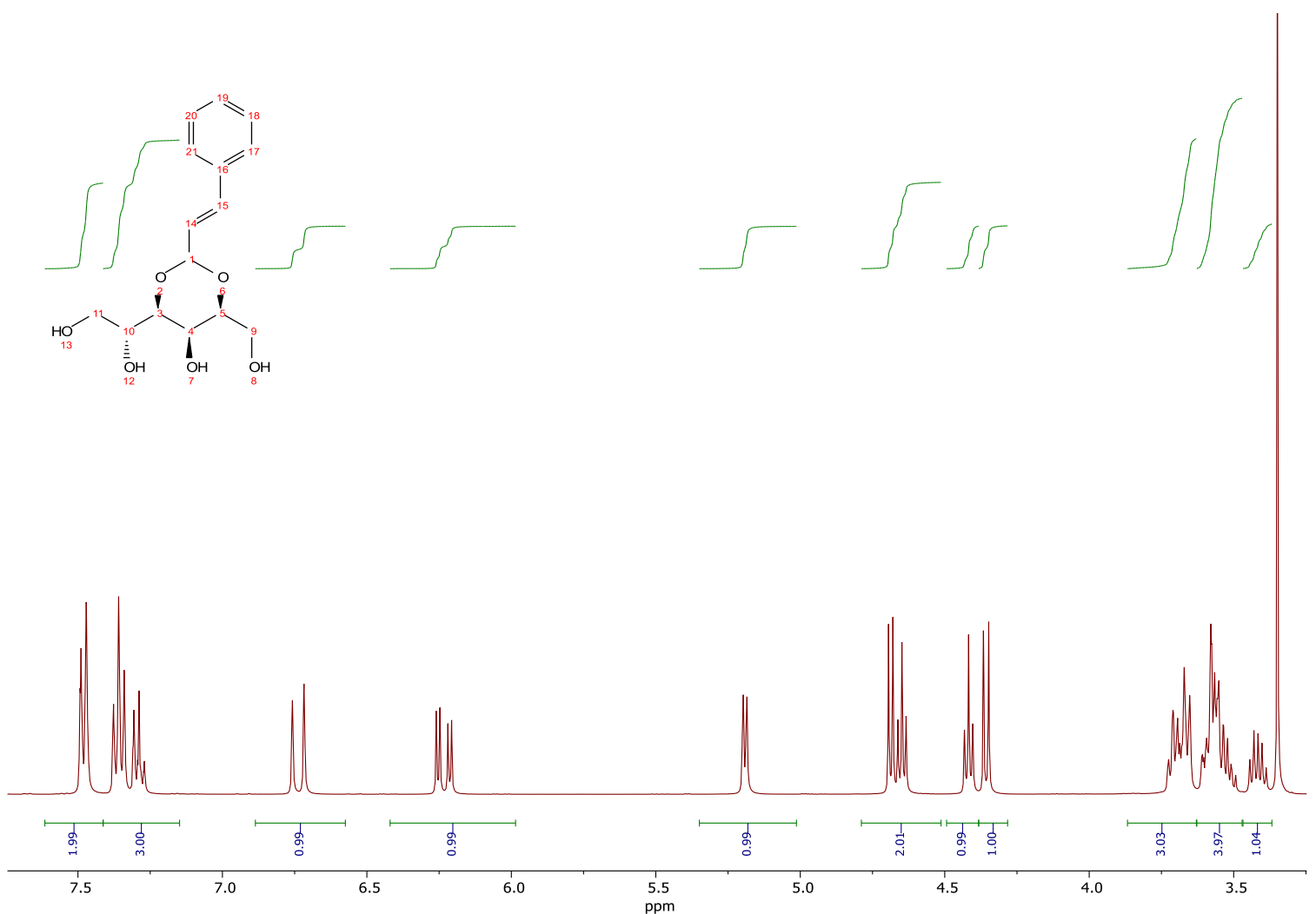

SI Fig. $15 \mid{ }^{1} \mathrm{H}$ NMR of MBS-Cinn 


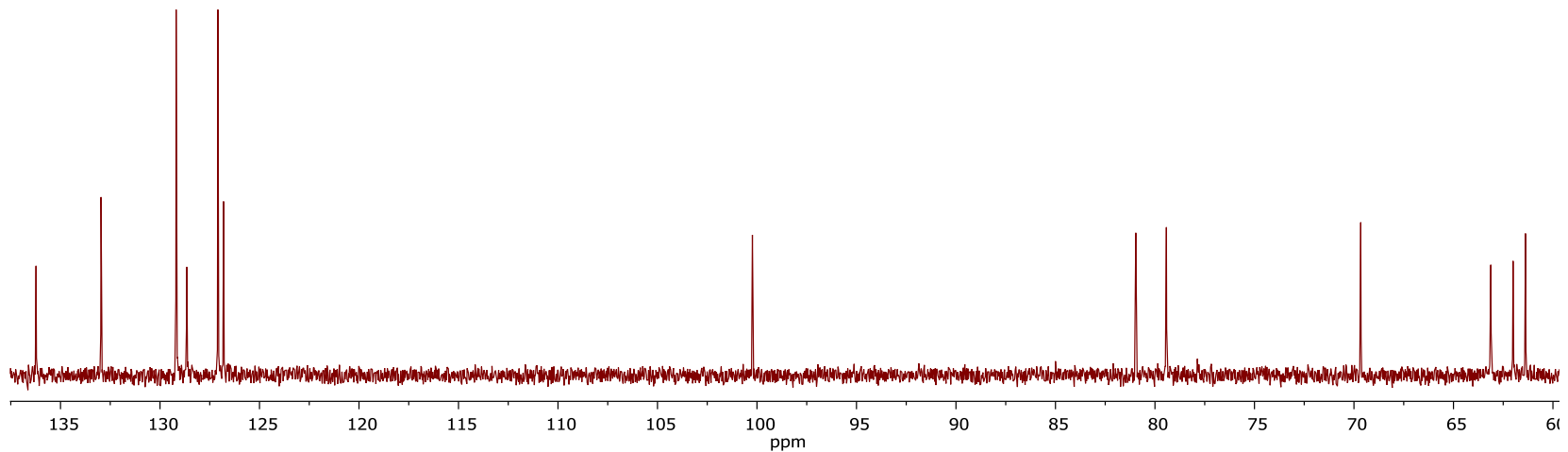

SI Fig. $16 \mid{ }^{13} \mathrm{C}$ NMR of MBS-Cinn

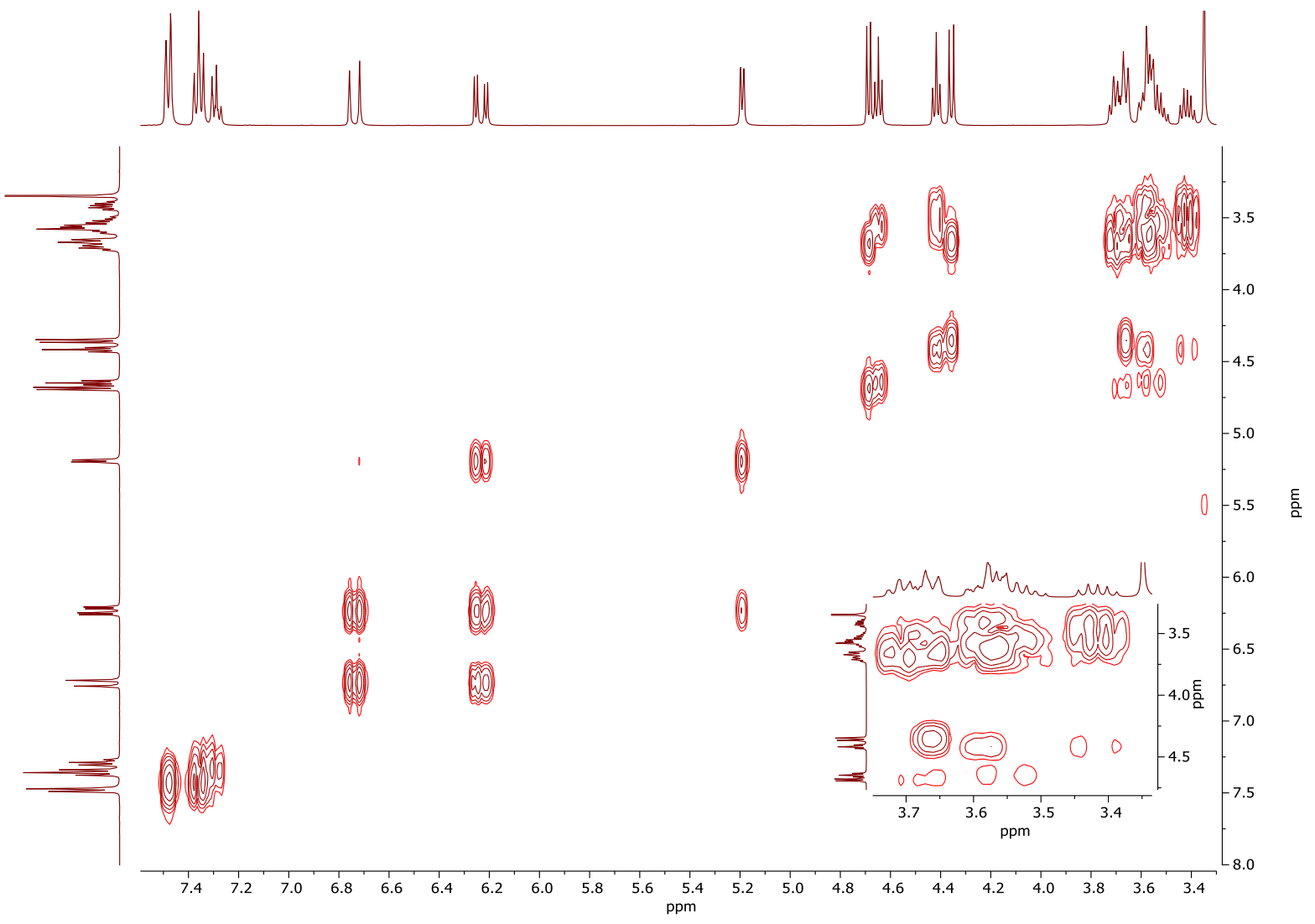

SI Fig. 17 | COSY of MBS-Cinn 


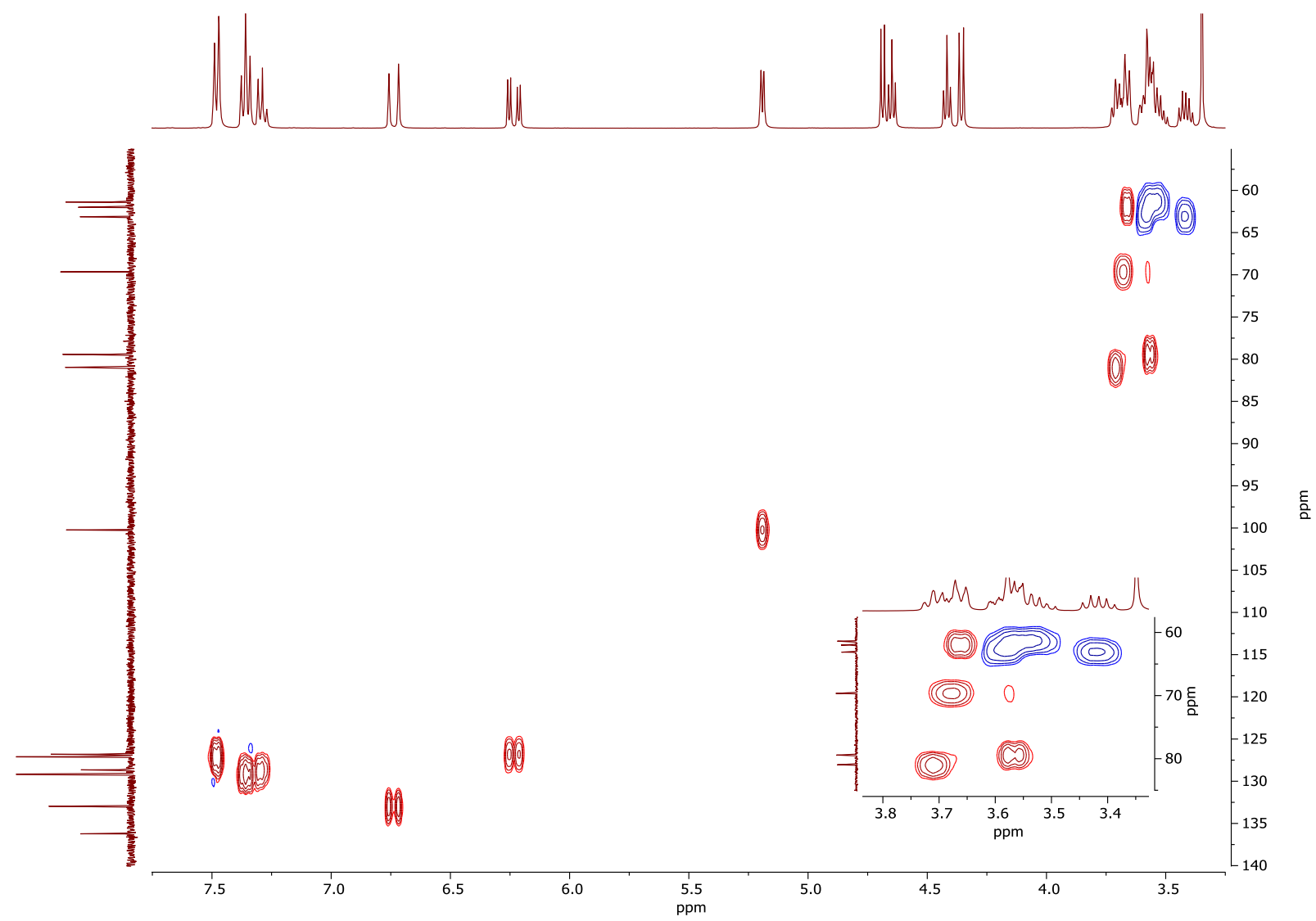

SI Fig. 18 | HSQC of MBS-Cinn

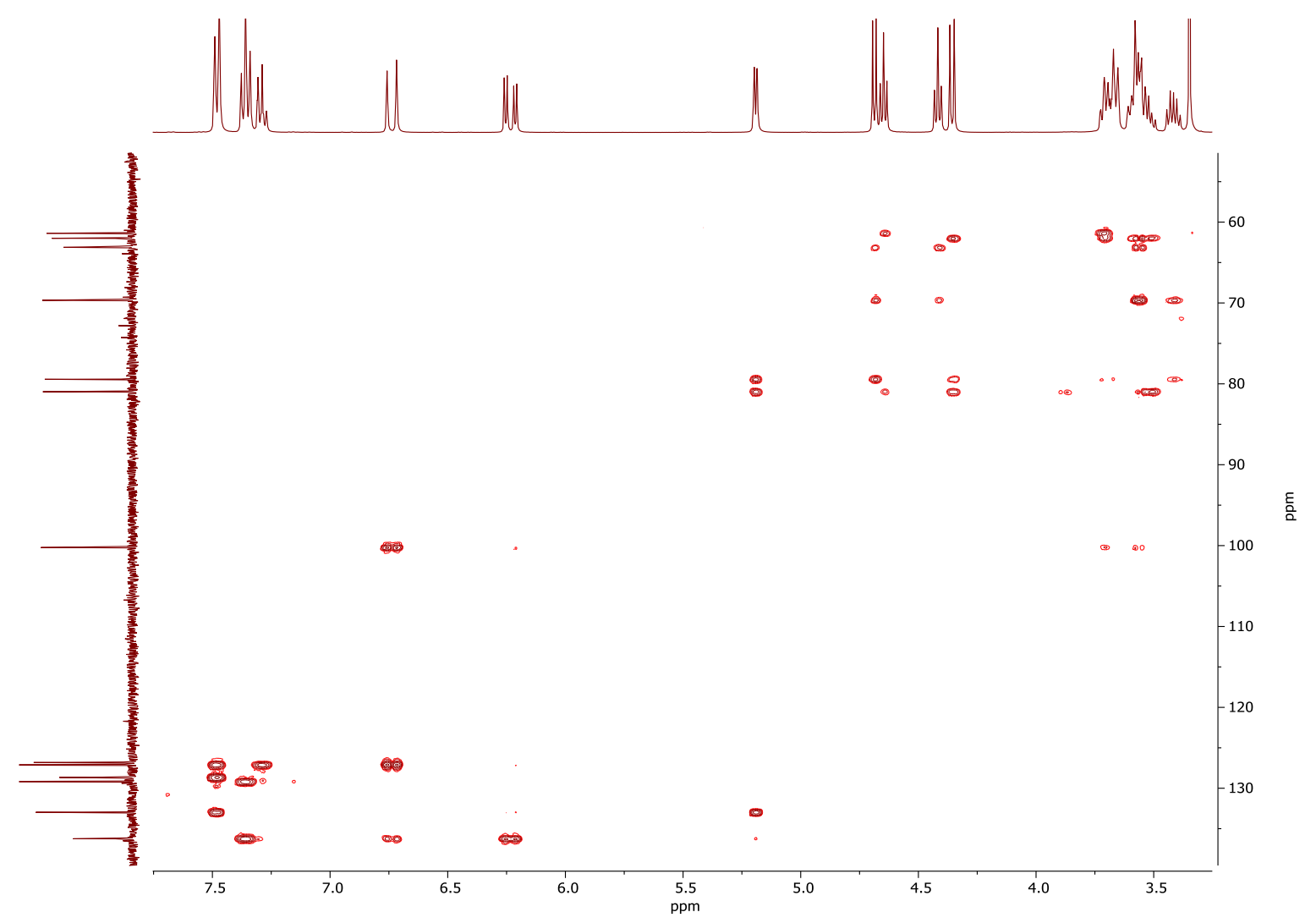

SI Fig. 19 | HMBC of MBS-Cin 


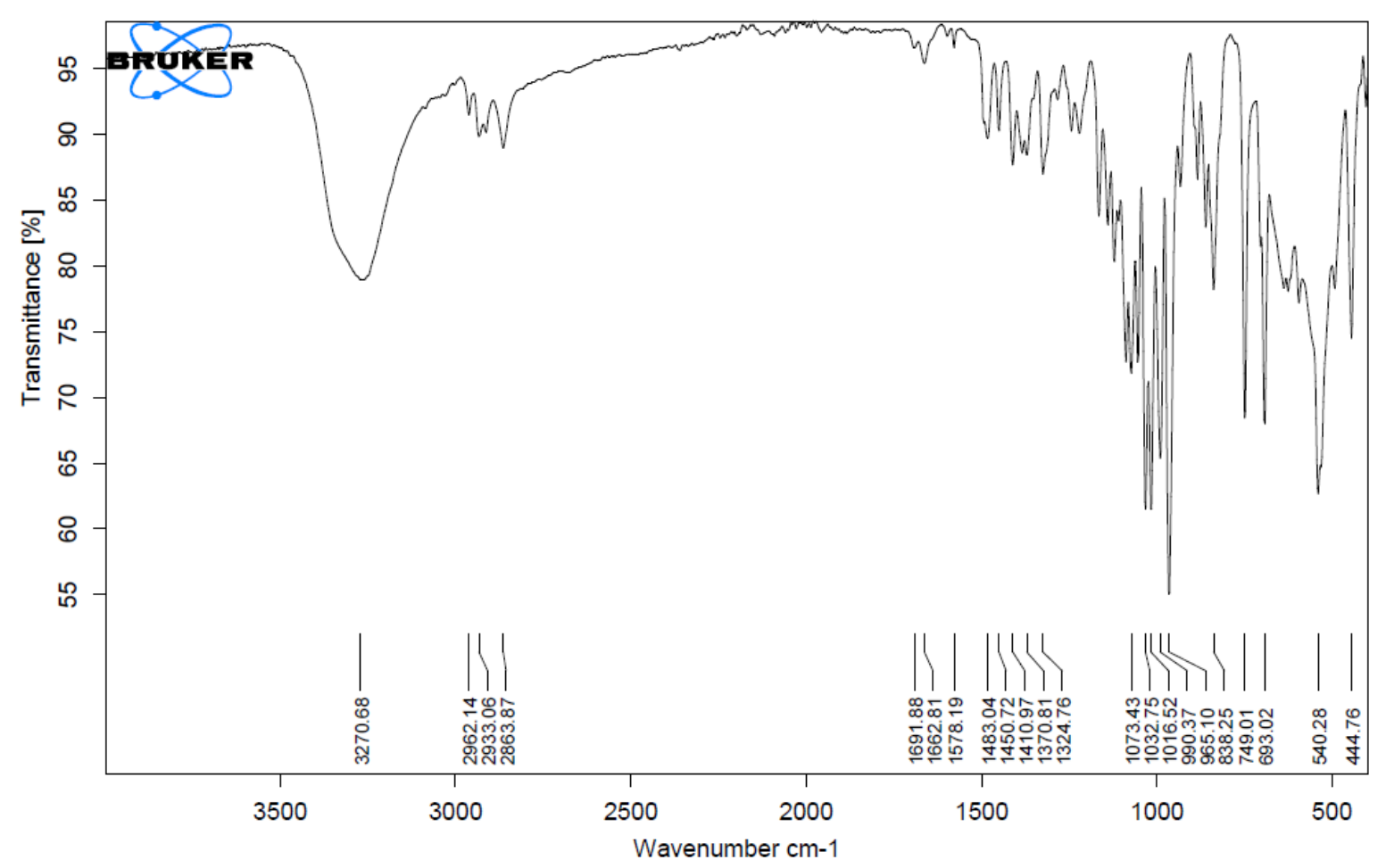

SI Fig. 20 | IR Spectrum of MBS-Cinn

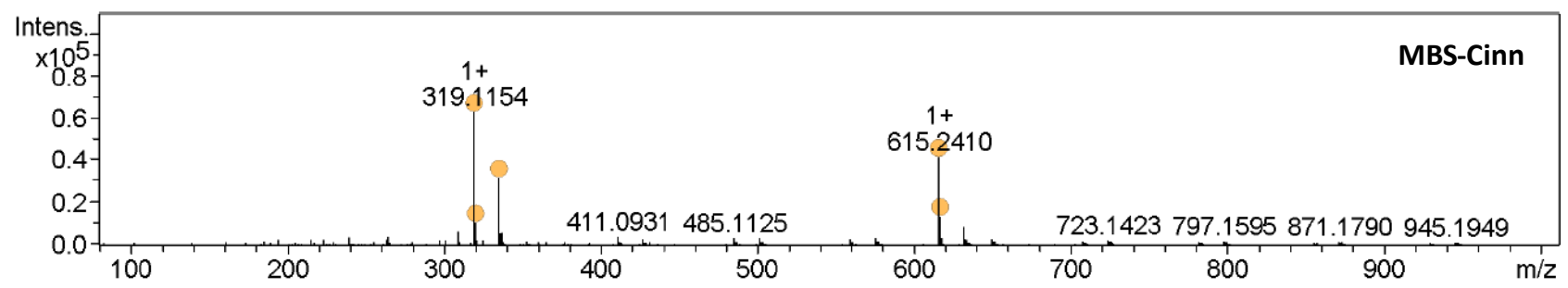

SI Fig. 21 | HRMS of MBS-Cinn 


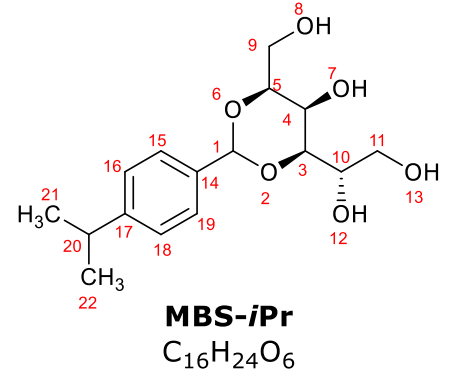

Synthesis of MBS-iPr: The titled compound was synthesized via general procedure to yield a white solid. Yield (59\%). Mp $131-133^{\circ} \mathrm{C}$. ${ }^{1} \mathrm{H}$ NMR $\left(400 \mathrm{MHz}, \mathrm{CD}_{3} \mathrm{OD}\right) \delta 7.48(2 \mathrm{H}, \mathrm{d}, J=8.1,15-\underline{H}, 19-\underline{H}), 7.23(2 \mathrm{H}$, $\mathrm{d}, J=8.1,16-\underline{H}, 18-\underline{H}), 5.62(1 \mathrm{H}, \mathrm{s}, 1-\underline{H}), 3.97(1 \mathrm{H}, \mathrm{ddd}, J=6.4,5.7,1.4$, 5- $\underline{H}), 3.91(1 \mathrm{H}, \mathrm{ddd}, 8.8,5.1,2.9,10-\underline{H}), 3.87(1 \mathrm{H}, \mathrm{t}, J=1.4,4-\underline{H}), 3.85-$ $3.83(1 \mathrm{H}, \mathrm{m}, 3-\underline{H}), 3.83-3.76\left(3 \mathrm{H}, \mathrm{m}, 9-\underline{H}_{2}, 11-\underline{H}_{\mathrm{b}}\right), 3.68(1 \mathrm{H}, \mathrm{dd}, J=$ $\left.11.5,5.2,11-\underline{H}_{a}\right), 2.92(1 \mathrm{H}$, hept, $J=6.9,20-\underline{H}), 1.25(6 \mathrm{H}, \mathrm{d}, J=6.9,21-$ $\left.\underline{H}_{3}, 22-\underline{H}_{3}\right) .{ }^{13} \mathrm{CNMR}\left(100 \mathrm{MHz}, \mathrm{CD}_{3} \mathrm{OD}\right) \delta 149.3$ (C14), 136.0 (C17), 126.2 (C19, C15), 125.5 (C18, C16), 101.2 (C1), 81.0 (C5), 79.4 (C3), 69.3 (C10), 62.8 (C11), 62.5 (C4), 61.7 (C9), 33.8 (C20), 23.0 (C21, C22). $v_{\max } / \mathrm{cm}^{-1} 3282 \mathrm{br}$ (OH sugar), 2941w, $2868 w, 1402 w, 1098 s, 1017 s$. (ESI) $\mathrm{m} / z(\mathrm{M}+\mathrm{Na})^{+}$calcd. for $\mathrm{C}_{16} \mathrm{H}_{24} \mathrm{NaO}_{6}{ }^{+} 355.1465$, found 335.1454 . $[\alpha]^{25} \mathrm{D}=+41.0$ (c. $\left.10.0 \mathrm{mg} \mathrm{mL}^{-1}, \mathrm{MeOH}\right)$. CHN Analysis: Calcd (\%) C 61.51; H 7.75; O 30.74; Found (100\%) C 61.51, H 7.79, 030.70 .

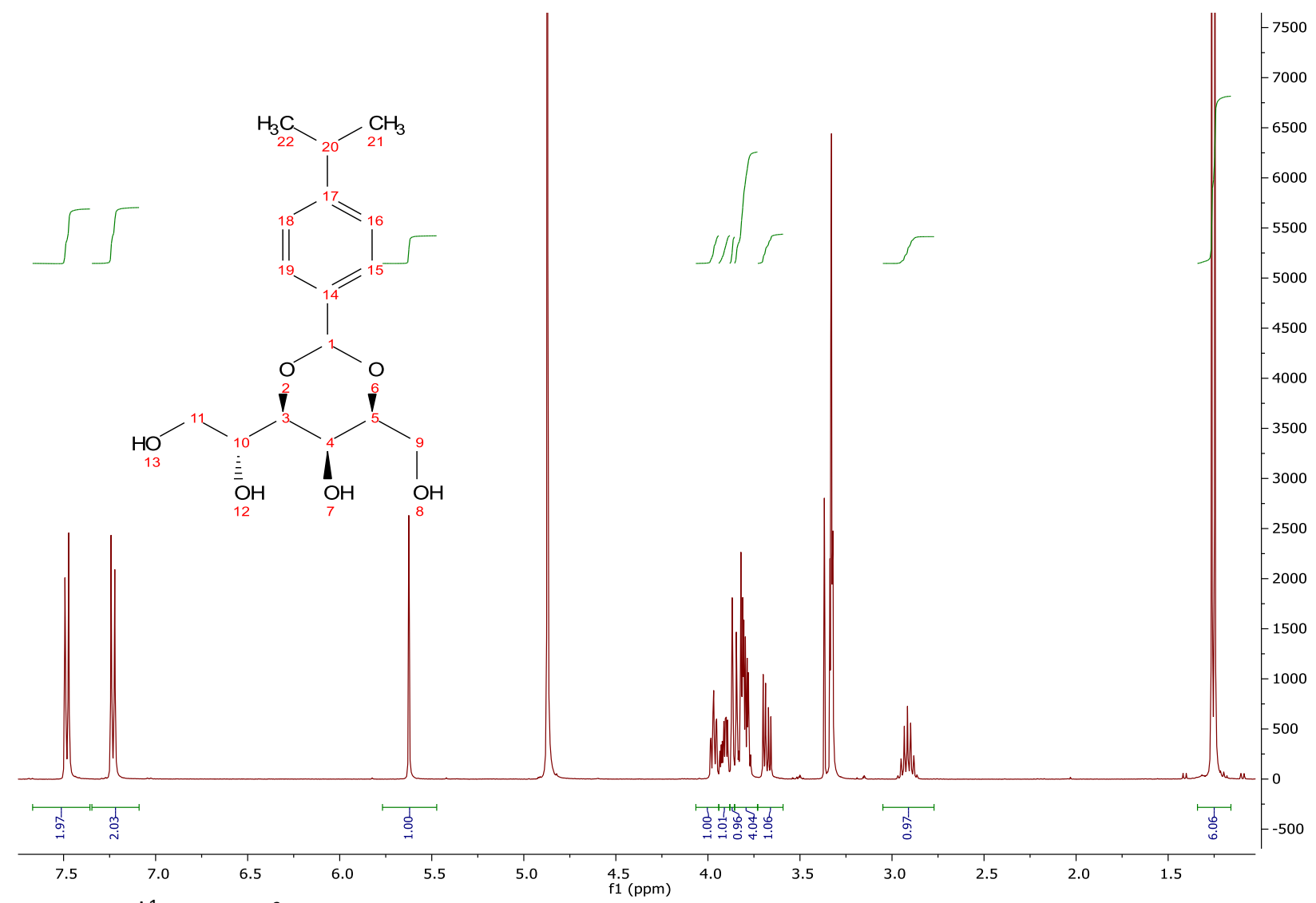

SI Fig. $\left.22\right|^{1} \mathrm{H}$ NMR of MBS-iPr 


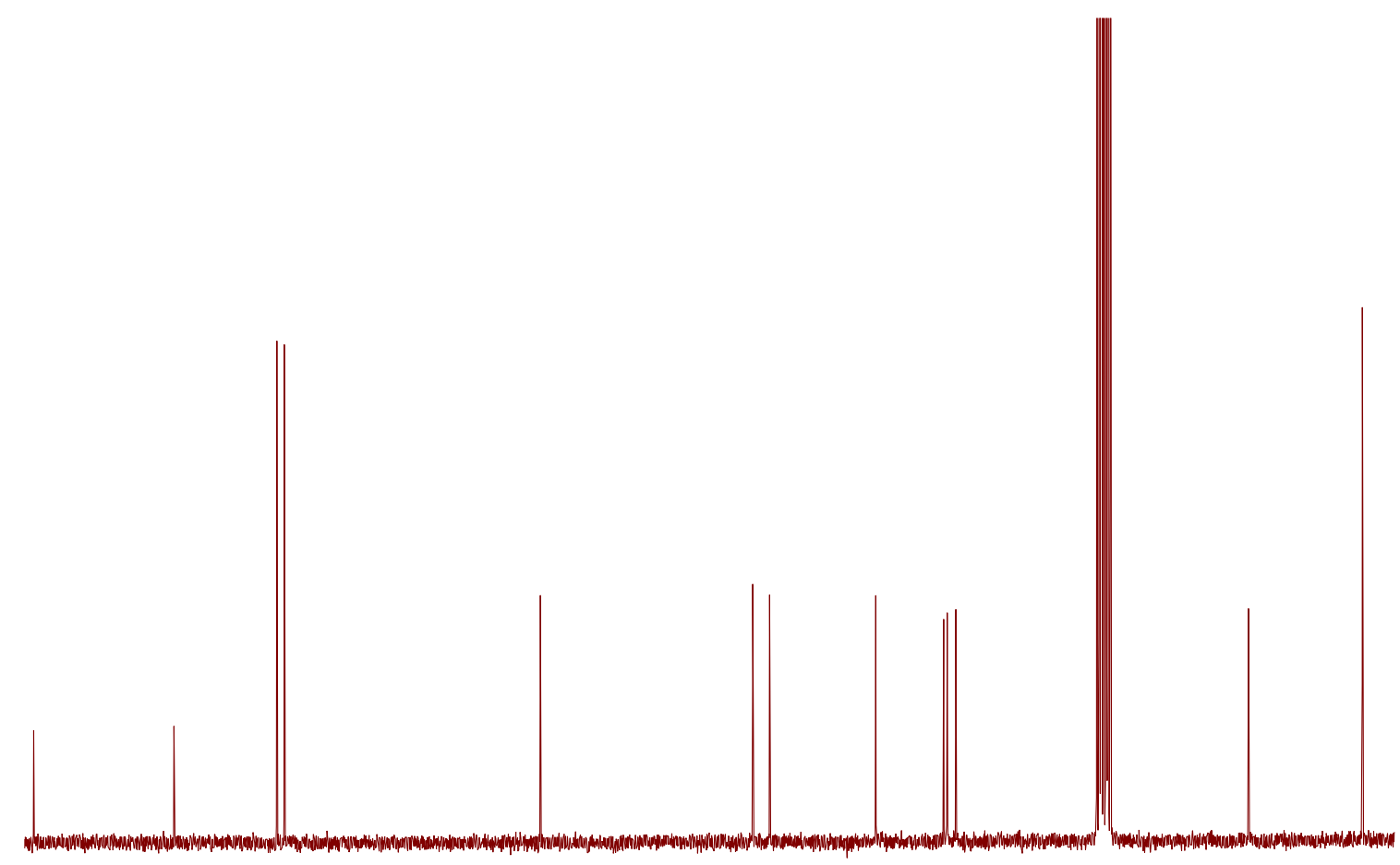

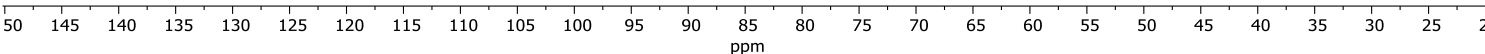
SI Fig. $23 \mid{ }^{13} \mathrm{C}$ NMR of MBS-iPr

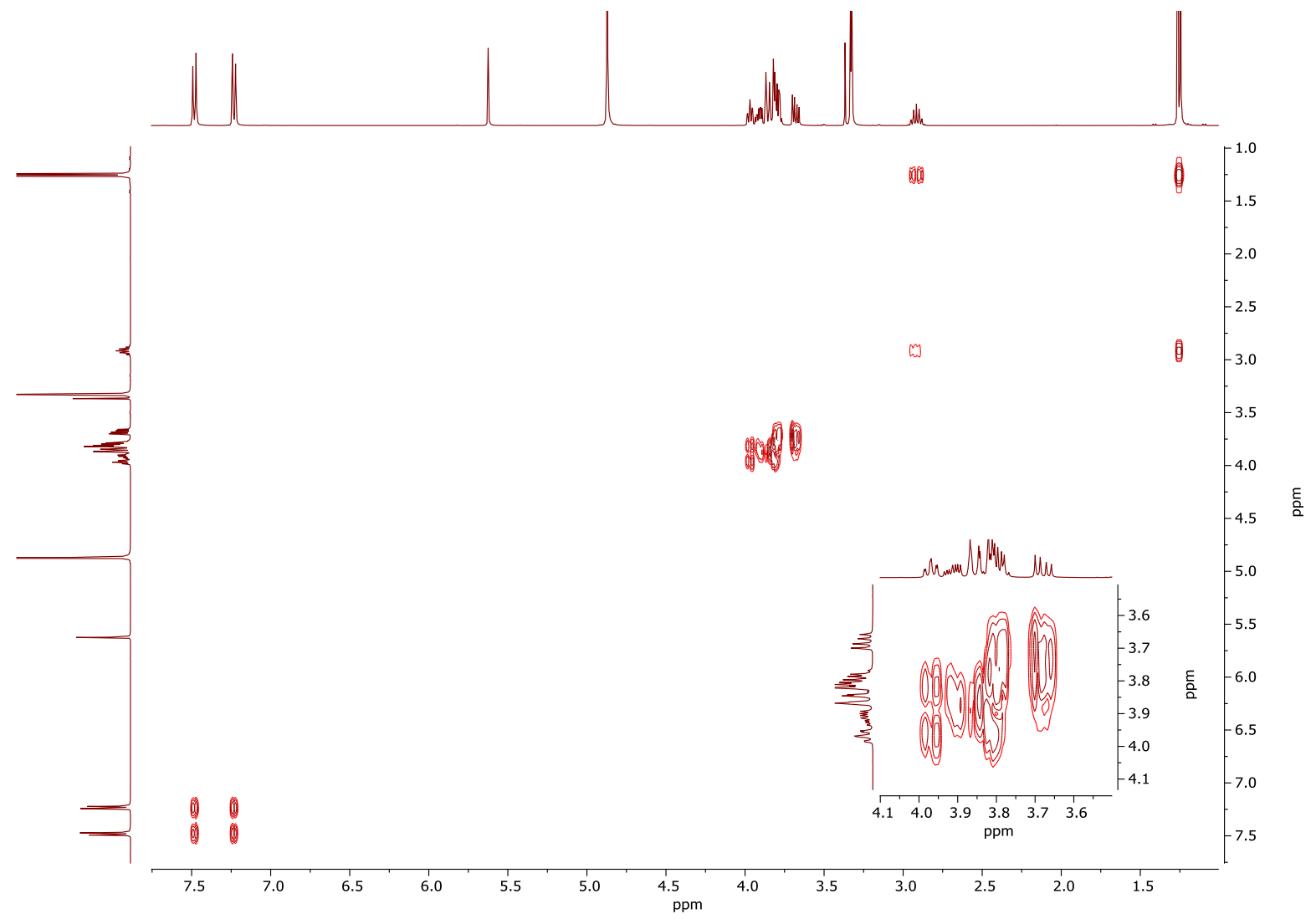

ESI Fig. 24 |COSY of MBS-iPr 


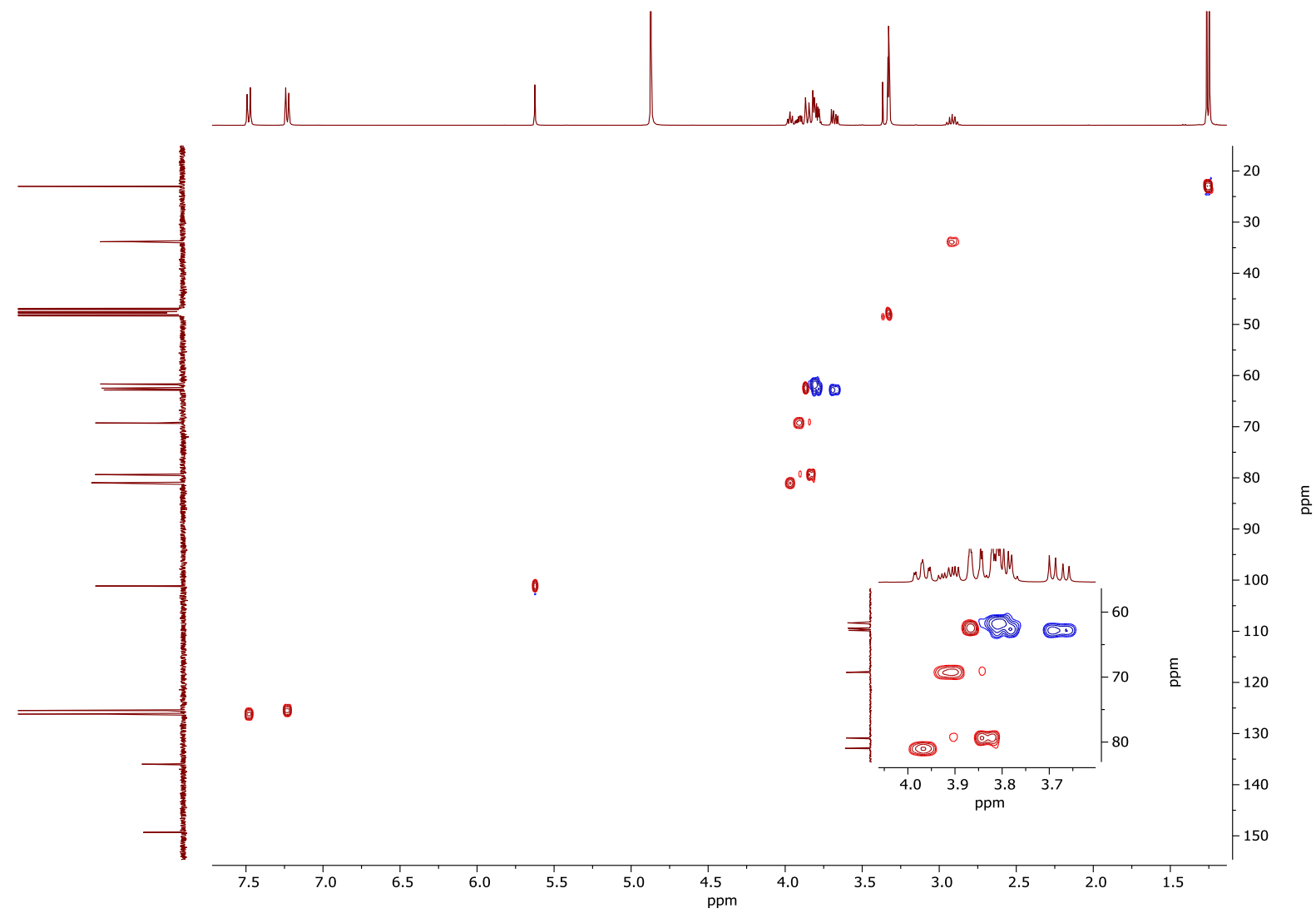

SI Fig. 25 |HSQC of MBS-iPr

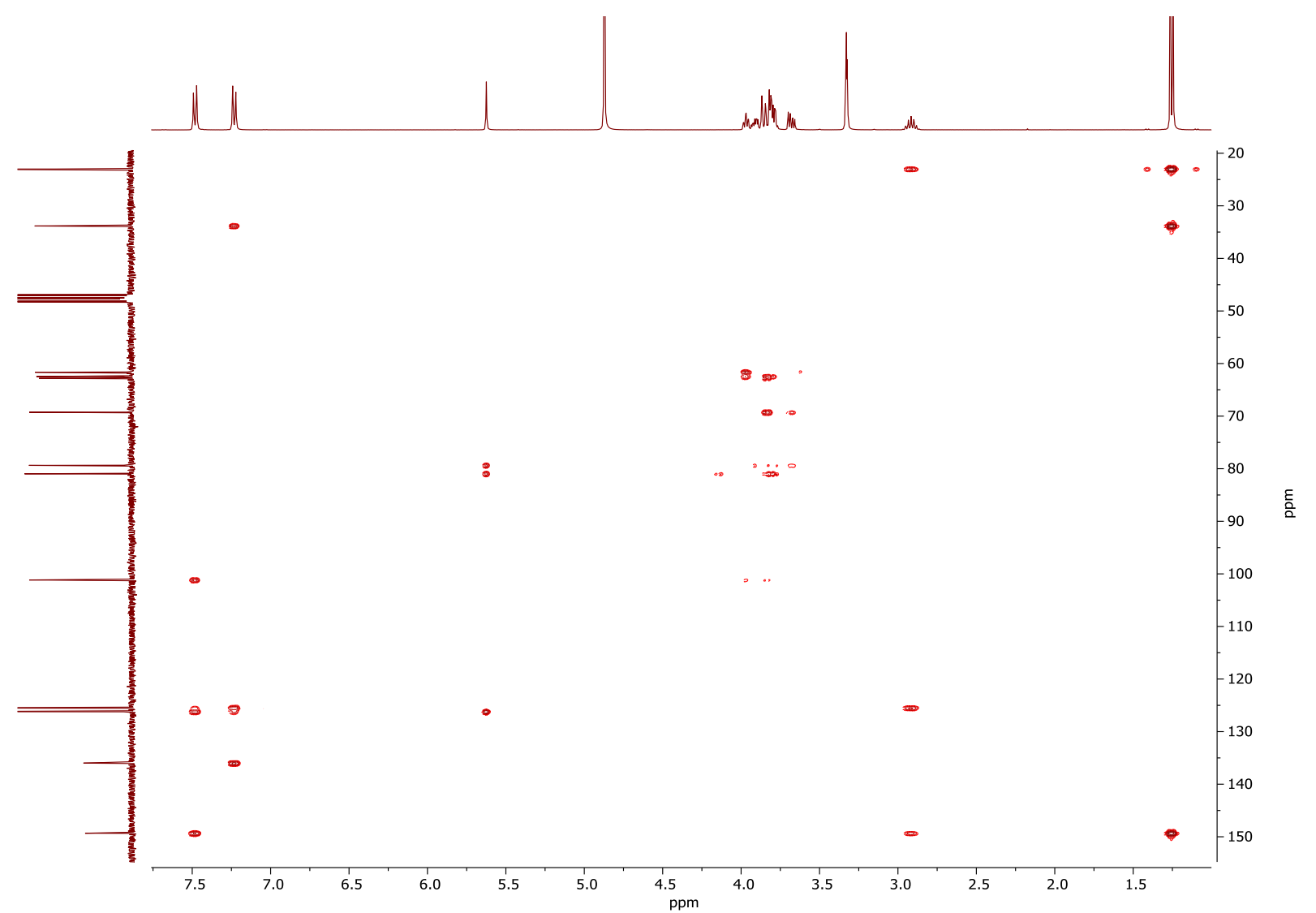

SI Fig. 26 |HMBC of MBS-iPr 


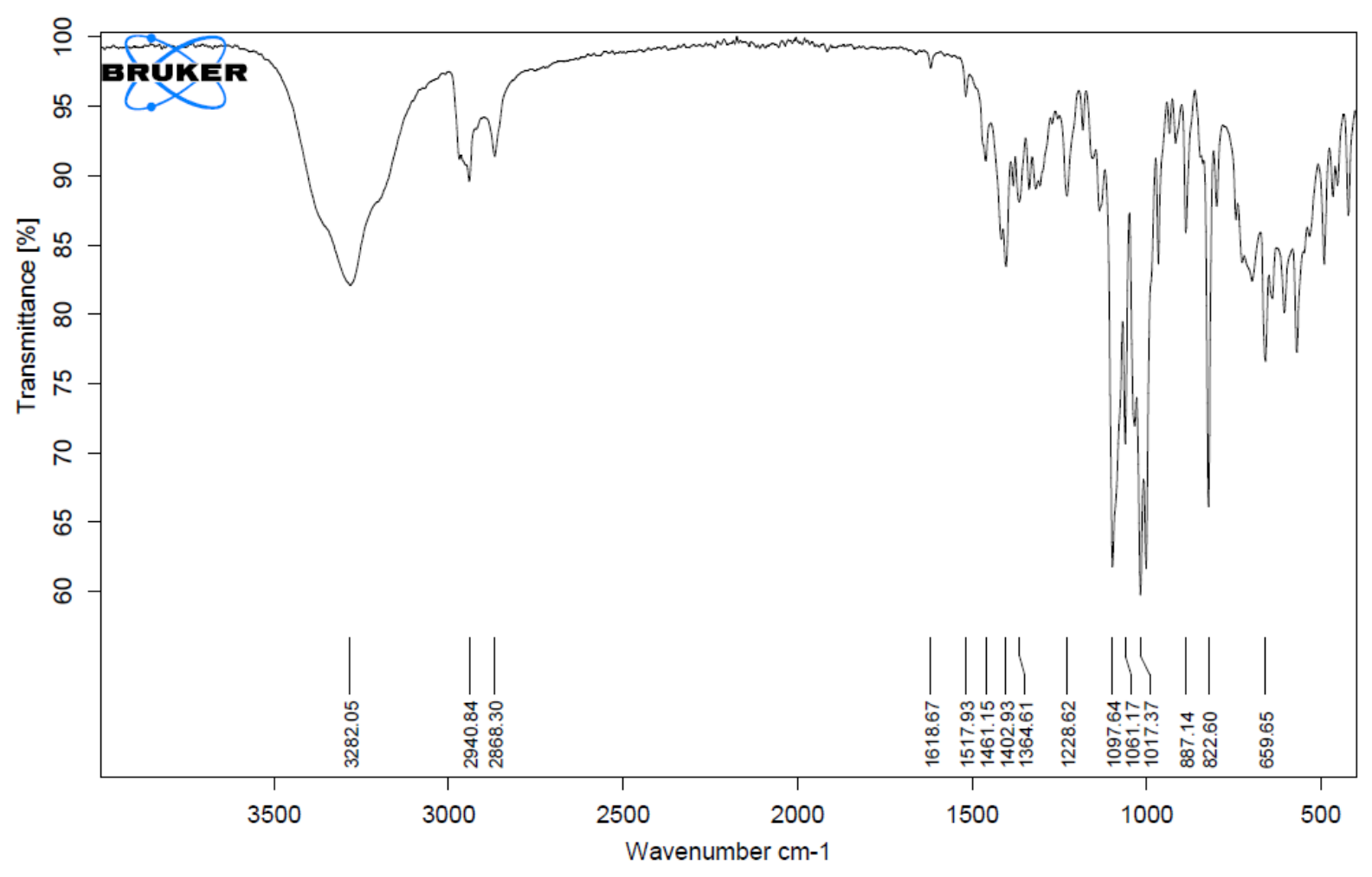

SI Fig. 27 | IR spectrum of MBS-iPr

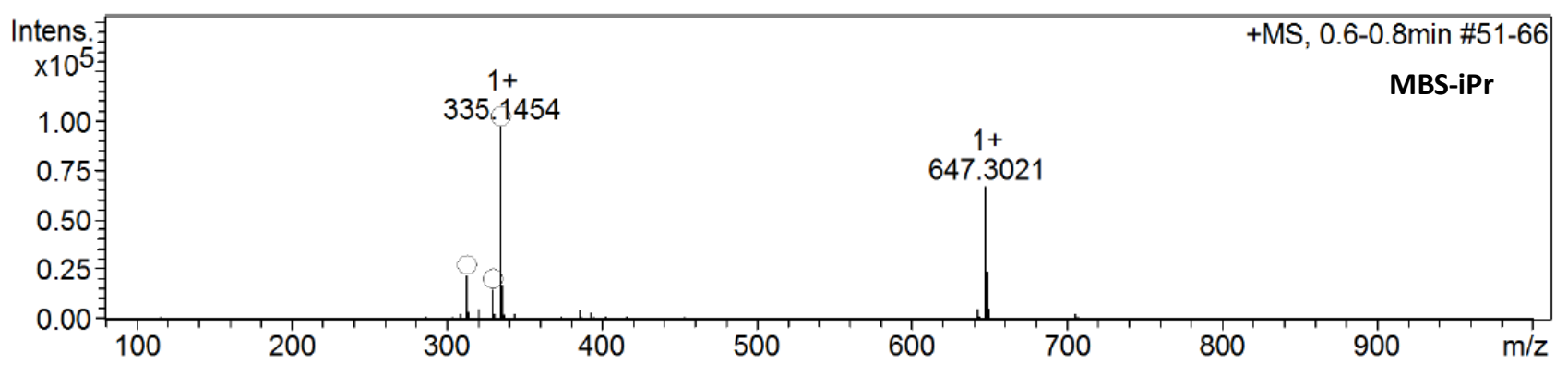

SI Fig. 28 | HRMS of MBS-iPr 


\begin{tabular}{|c|c|c|c|c|c|c|c|}
\hline \multirow{11}{*}{ 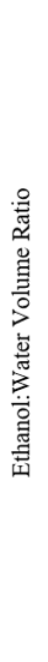 } & $\mathrm{EtOH}$ & $\mathrm{S}$ & $\mathrm{P}$ & P & $\mathrm{G}(\mathrm{O})$ & $\mathrm{G}(\mathrm{O})$ & $\mathrm{G}(\mathrm{O})$ \\
\hline & $90: 10$ & $\mathrm{~S}$ & $P$ & $P$ & $\mathrm{G}(\mathrm{O})$ & $\mathrm{G}(\mathrm{O})$ & $\mathrm{G}(\mathrm{O})$ \\
\hline & $80: 20$ & $\mathrm{~S}$ & $\mathrm{P}$ & $\mathrm{G}(\mathrm{O})$ & $\mathrm{G}(\mathrm{O})$ & $\mathrm{G}(\mathrm{O})$ & $\mathrm{G}(\mathrm{O})$ \\
\hline & $70: 30$ & $P G(T)$ & $\mathrm{G}(\mathrm{O})$ & $\mathrm{G}(\mathrm{O})$ & $\mathrm{G}(\mathrm{O})$ & $\mathrm{G}(\mathrm{O})$ & $\mathrm{G}(\mathrm{O})$ \\
\hline & $60: 40$ & $\mathrm{G}(\mathrm{T})$ & $\mathrm{G}(\mathrm{O})$ & $\mathrm{G}(\mathrm{O})$ & $\mathrm{G}(\mathrm{O})$ & $\mathrm{G}(\mathrm{O})$ & $\mathrm{G}(\mathrm{O})$ \\
\hline & $50: 50$ & $\mathrm{G}(\mathrm{T})$ & $\mathrm{G}(\mathrm{O})$ & $\mathrm{G}(\mathrm{O})$ & $\mathrm{G}(\mathrm{O})$ & $\mathrm{G}(\mathrm{O})$ & $\mathrm{G}(\mathrm{O})$ \\
\hline & $40: 60$ & $\mathrm{G}(\mathrm{T})$ & $\mathrm{G}(\mathrm{O})$ & $\mathrm{G}(\mathrm{O})$ & $\mathrm{G}(\mathrm{O})$ & $\mathrm{G}(\mathrm{O})$ & $\mathrm{G}(\mathrm{O})$ \\
\hline & $30: 70$ & $\mathrm{G}(\mathrm{T})^{\mathrm{b}}$ & $\mathrm{G}(\mathrm{O})$ & $\mathrm{G}(\mathrm{O})$ & $\mathrm{G}(\mathrm{O})$ & $\mathrm{G}(\mathrm{O})^{\mathrm{a}}$ & $\mathrm{G}(\mathrm{O})^{\mathrm{a}}$ \\
\hline & $20: 80$ & $G(T)^{b}$ & $\mathrm{G}(\mathrm{O})$ & $\mathrm{G}(\mathrm{O})$ & $\mathrm{G}(\mathrm{O})^{\mathrm{a}}$ & $\mathrm{G}(\mathrm{O})^{\mathrm{a}}$ & $\mathrm{G}(\mathrm{O})^{\mathrm{a}}$ \\
\hline & $10: 90$ & $P G(T)^{a}$ & $G(T)^{a}$ & $\mathrm{G}(\mathrm{O})$ & $\mathrm{G}(\mathrm{O})^{\mathrm{a}}$ & $\mathrm{G}(\mathrm{O})^{\mathrm{a}}$ & $\mathrm{G}(\mathrm{O})^{\mathrm{a}}$ \\
\hline & $\mathrm{H}_{2} \mathrm{O}$ & I & I & I & I & I & I \\
\hline & & 1 & 3 & 5 & 7 & 10 & 15 \\
\hline
\end{tabular}

\begin{tabular}{|c|c|c|c|c|c|c|c|c|}
\hline \multirow{6}{*}{ 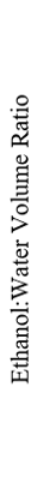 } & $50: 50$ & $\mathrm{~S}$ & $\mathrm{~S}$ & $\mathrm{~S}$ & $\mathrm{~S}$ & $\mathrm{~S}$ & $\mathrm{~S}$ & P \\
\hline & $40: 60$ & $\mathrm{~S}$ & $\mathrm{~S}$ & $\mathrm{~S}$ & $\mathrm{~S}$ & $\mathrm{P}$ & $\mathrm{P}$ & $\begin{array}{c}\mathrm{G} \\
(\mathrm{O})^{\mathrm{b}}\end{array}$ \\
\hline & $30: 70$ & S & S & $P$ & $\mathrm{G}(\mathrm{O})$ & $\mathrm{G}(\mathrm{O})$ & $\mathrm{G}(\mathrm{O})$ & $\mathrm{G}(\mathrm{O})$ \\
\hline & $20: 80$ & $S$ & S & $P$ & $\mathrm{G}(\mathrm{O})$ & $\mathrm{G}(\mathrm{O})$ & $\mathrm{G}(\mathrm{O})$ & $\mathrm{G}(\mathrm{O})$ \\
\hline & $10: 90$ & S & S & $P$ & $\mathrm{G}(\mathrm{O})$ & $\mathrm{G}(\mathrm{O})$ & $\mathrm{G}(\mathrm{O})$ & $\mathrm{G}(\mathrm{O})$ \\
\hline & $\mathrm{H}_{2} \mathrm{O}$ & S & $\begin{array}{l}\text { PG } \\
\text { (O) }\end{array}$ & $\mathrm{G}(\mathrm{O})$ & $\mathrm{G}(\mathrm{O})$ & $\mathrm{G}(\mathrm{O})$ & $\mathrm{G}(\mathrm{O})$ & $\mathrm{G}(\mathrm{O})$ \\
\hline & & 3 & 5 & 7 & 10 & 15 & 20 & 25 \\
\hline
\end{tabular}

SI Fig. 29 | DBS-iPr and MBS-iPr phase diagram. I = insoluble, $\mathrm{G}=$ gel, $\mathrm{S}=$ solution, $\mathrm{P}=$ precipitate, PG $=$ Partial Gel (PG), (T) =transparent, $(\mathrm{O})=$ opaque. 'gelation occurred overnight 


\section{Morphologies}
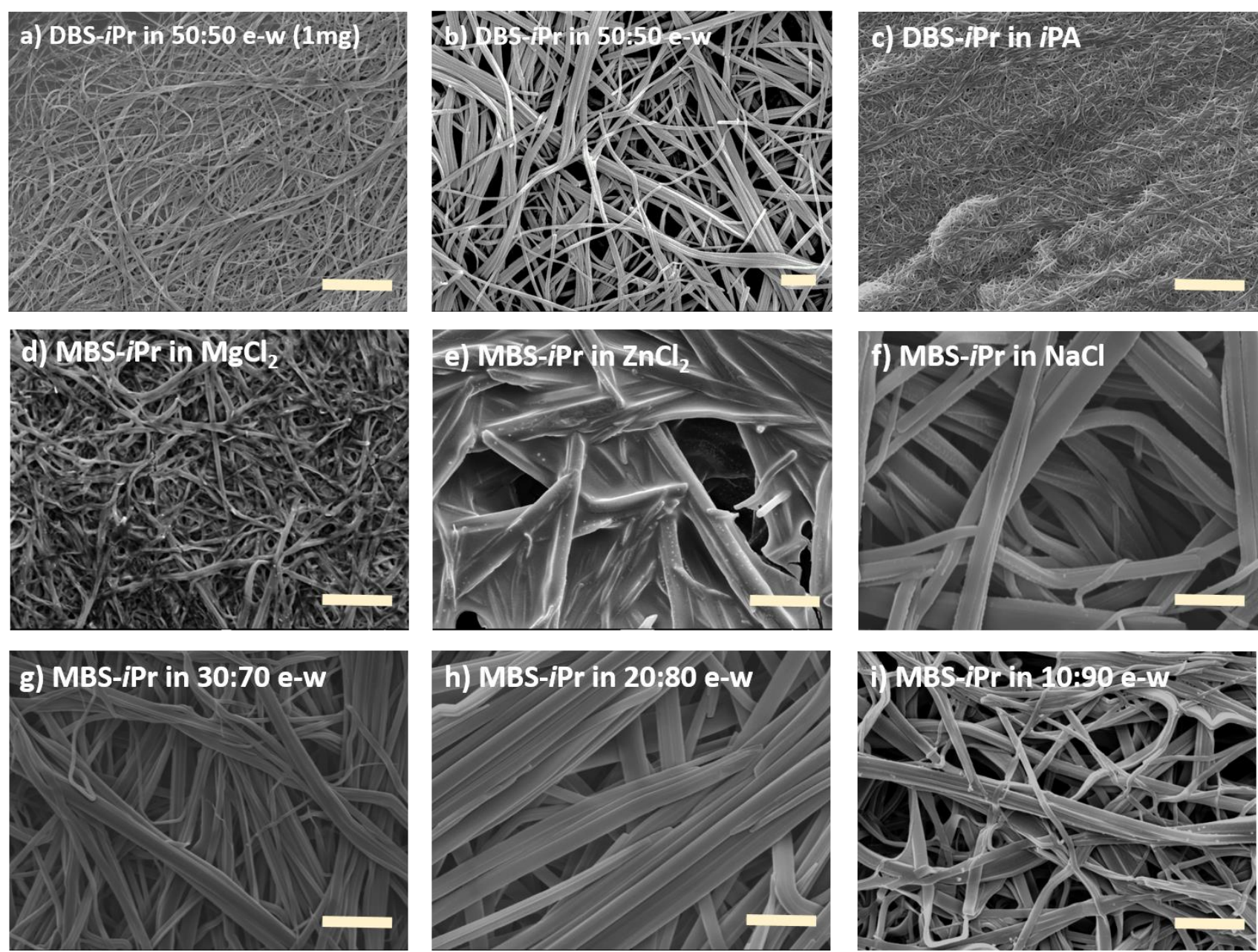

SI Fig. 30 | SEM micrographs of DBS-iPr and MBS-iPr xerogels formed in different solvents. All in $1 \%$ $\mathrm{w} / \mathrm{v}$ (except $\mathrm{a}-0.1 \% \mathrm{w} / \mathrm{v})$ upon heating and cooling. Conditions: xerogel prepared by drying the gel in air and then coating with $5 \mathrm{~nm}$ IR before imaging under vacuum at $5 \mathrm{kV}$. Scale bar in all images is $1 \mu \mathrm{m}$. 

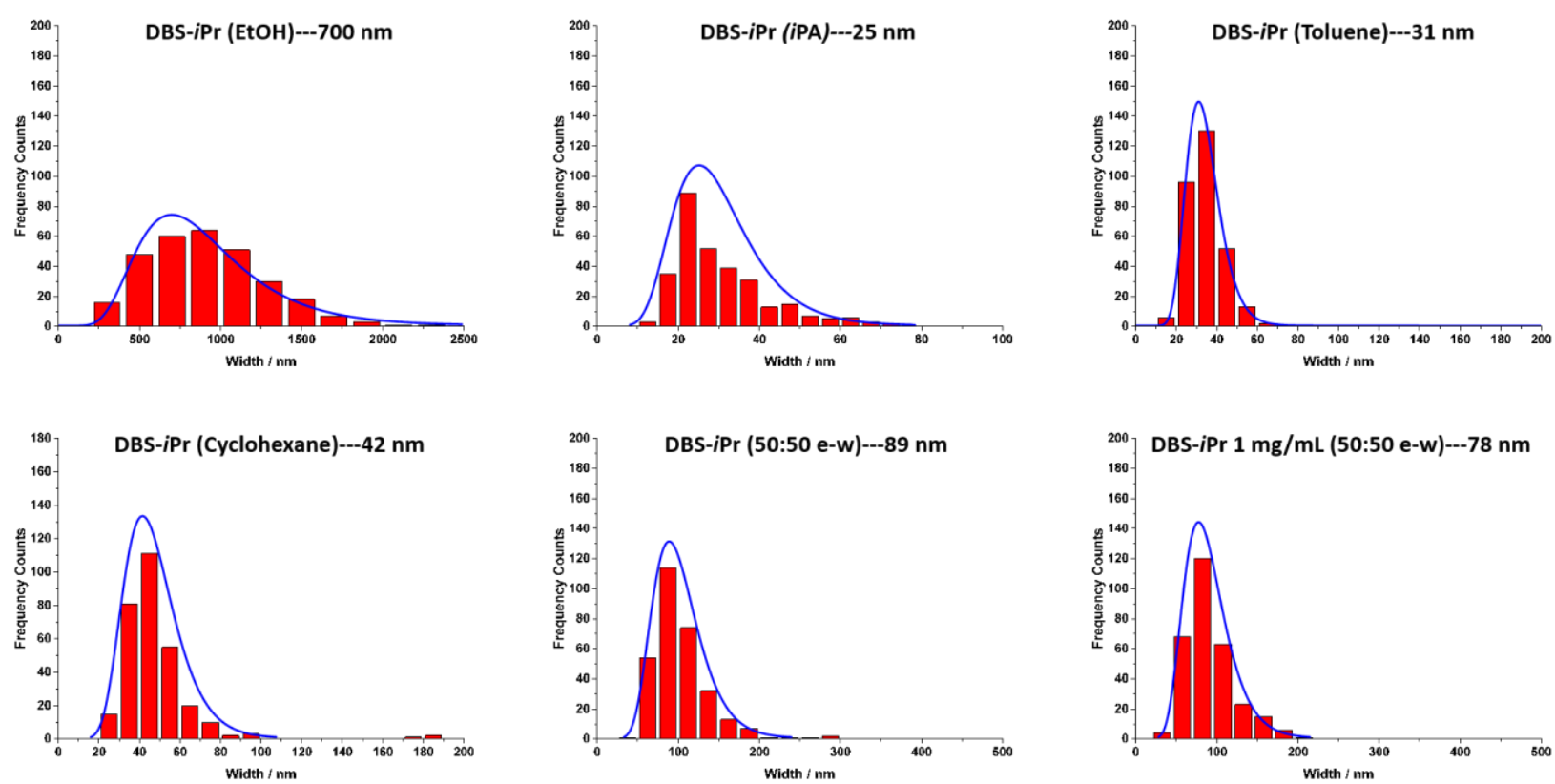

SI Fig. 31 | Distribution histograms for DBS-iPr xerogels width fibre dimensions from SEM images all at $10 \mathrm{mg} / \mathrm{mL}$ unless stated. 

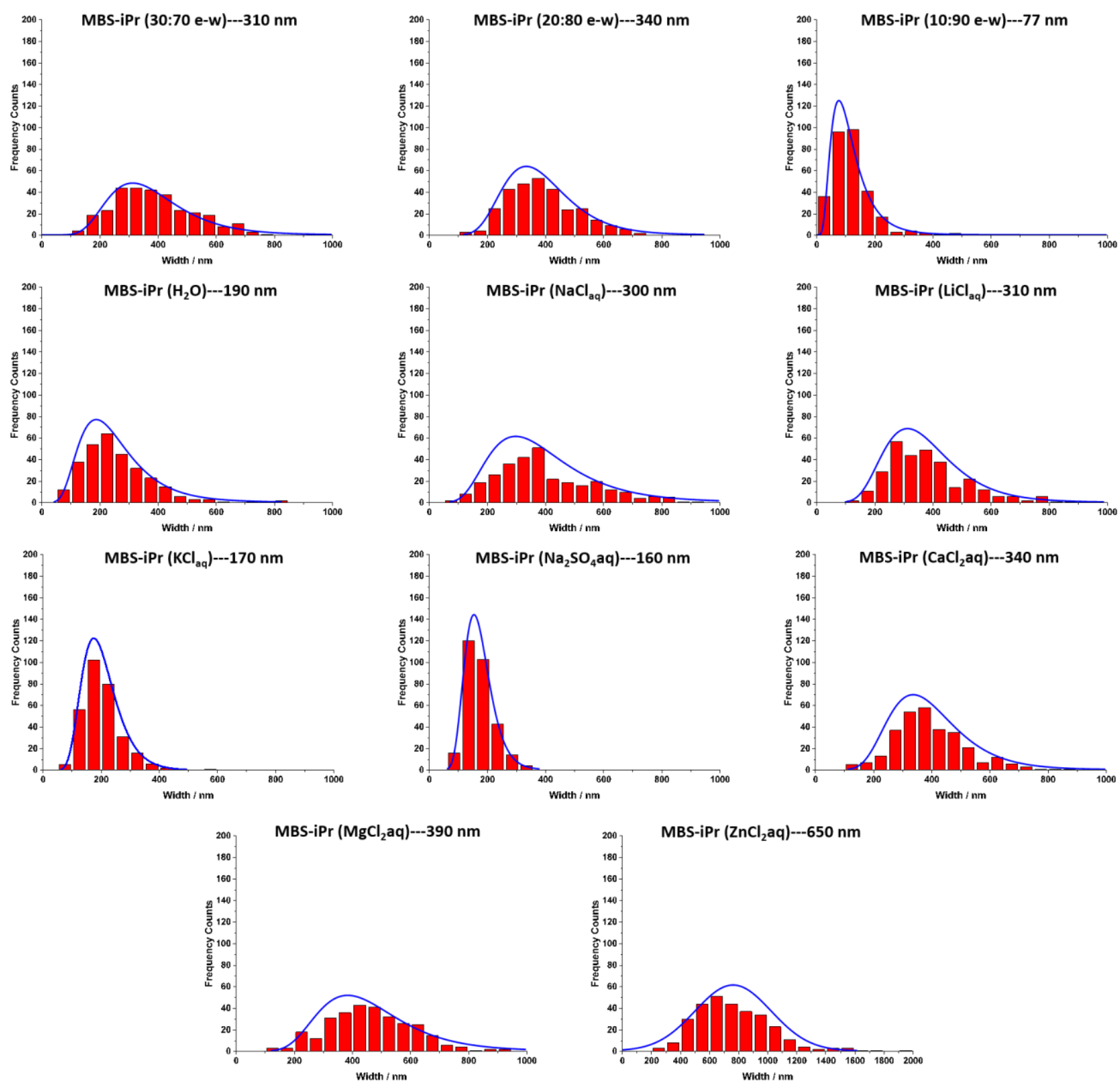

SI Fig. 32 | Distribution histograms for M78BS-iPr xerogels width fibre dimensions from SEM images all at $10 \mathrm{mg} / \mathrm{mL}$ 

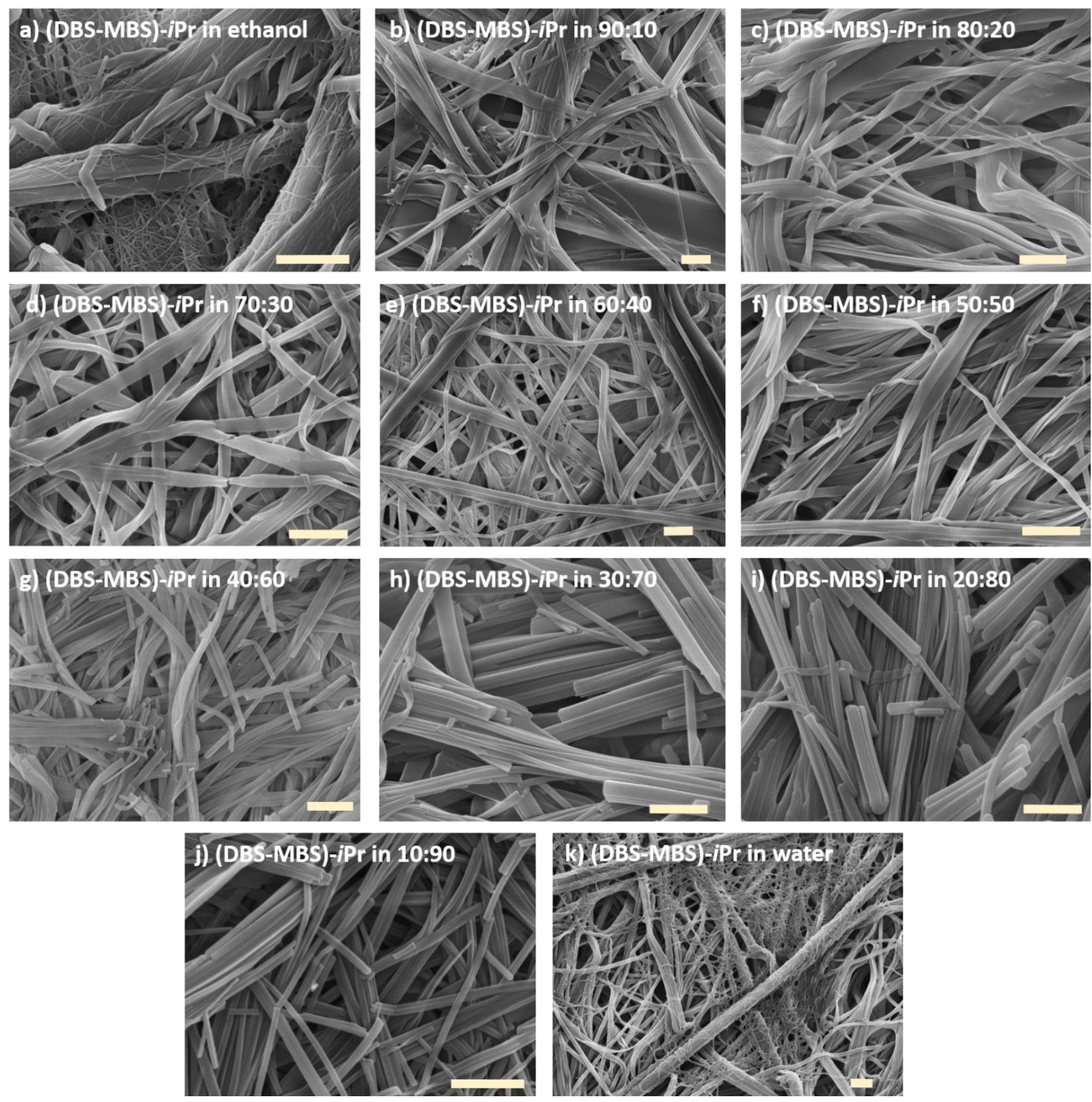

SI Fig. 33 | SEM micrographs of equimolar xerogels formed in different solvents. All in $1 \% \mathrm{w} / \mathrm{v}$ upon heating and cooling. Conditions: xerogel prepared by drying the gel in ain and then coating with $5 \mathrm{~nm}$ IR before imaging under vacuum at $5 \mathrm{kV}$. Scale bar in all images is $1 \mu \mathrm{m}$. 
Powder X-Ray Diffraction

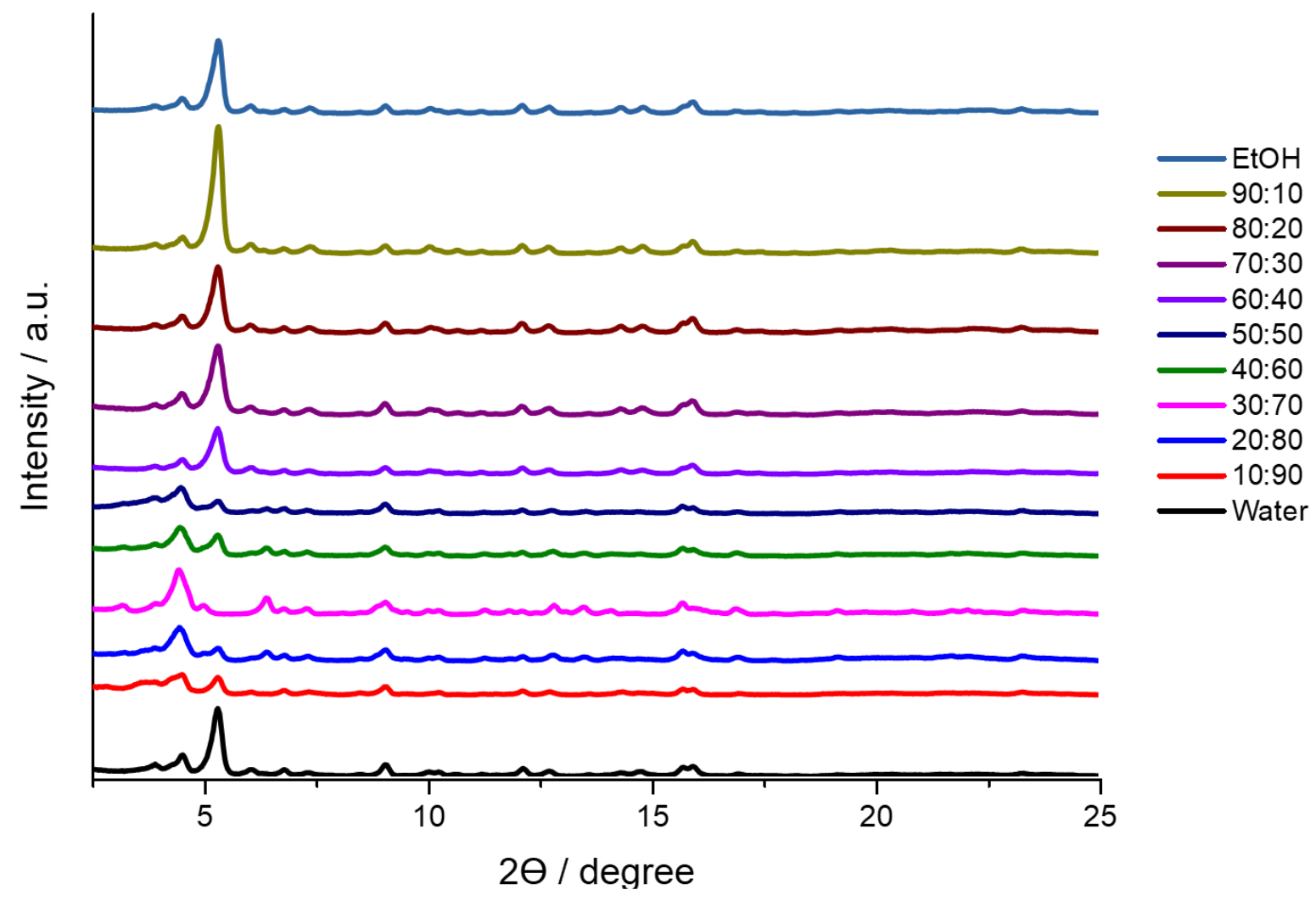

SI Fig. 34 | PXRD of equimolar xerogel made from all ethanolic/water solutions

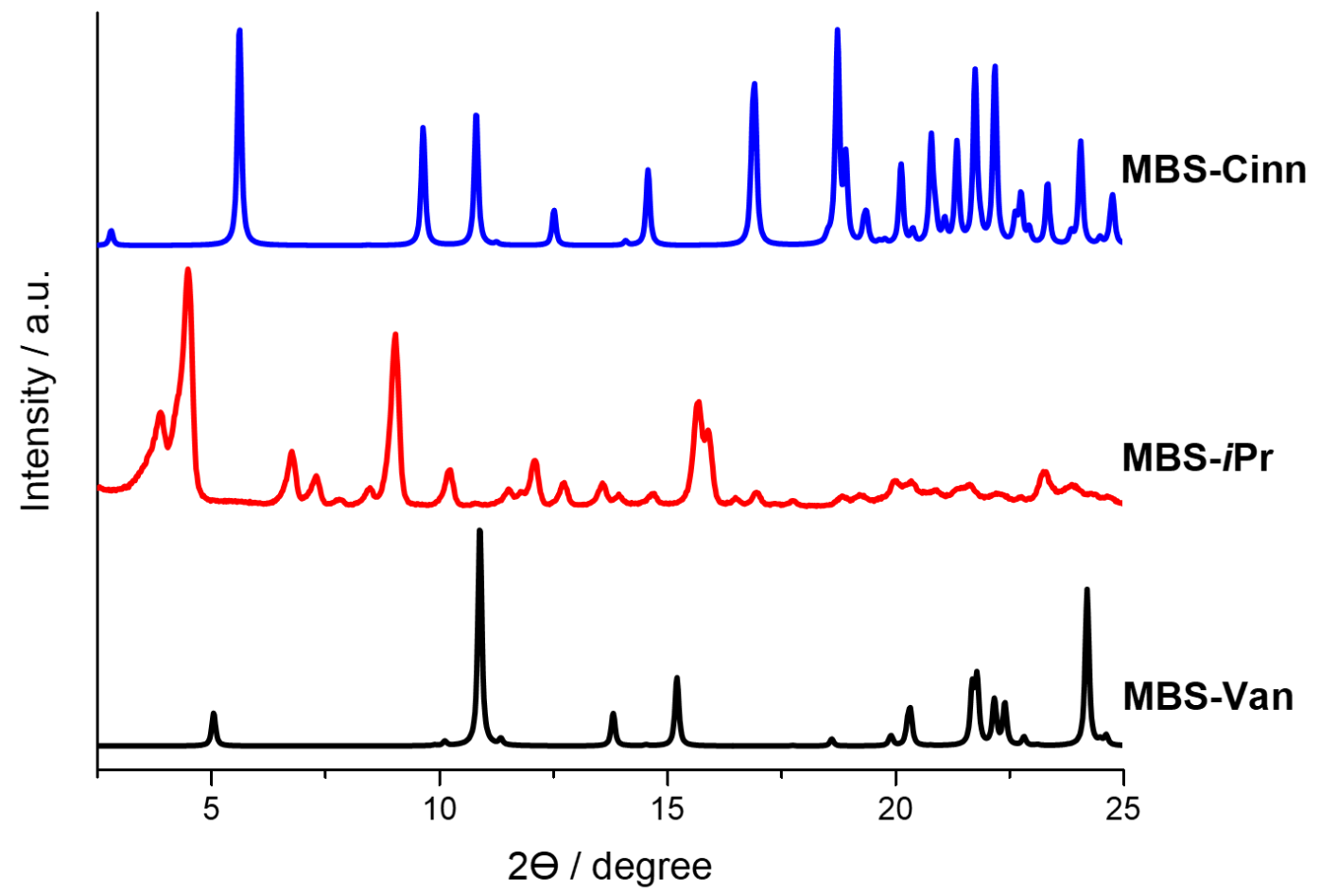

SI Fig. 35 | MBS-iPr PXRD spectrum and the simulated PXRD of MBS-Cinn and MBS-Van from single crystal diffraction 

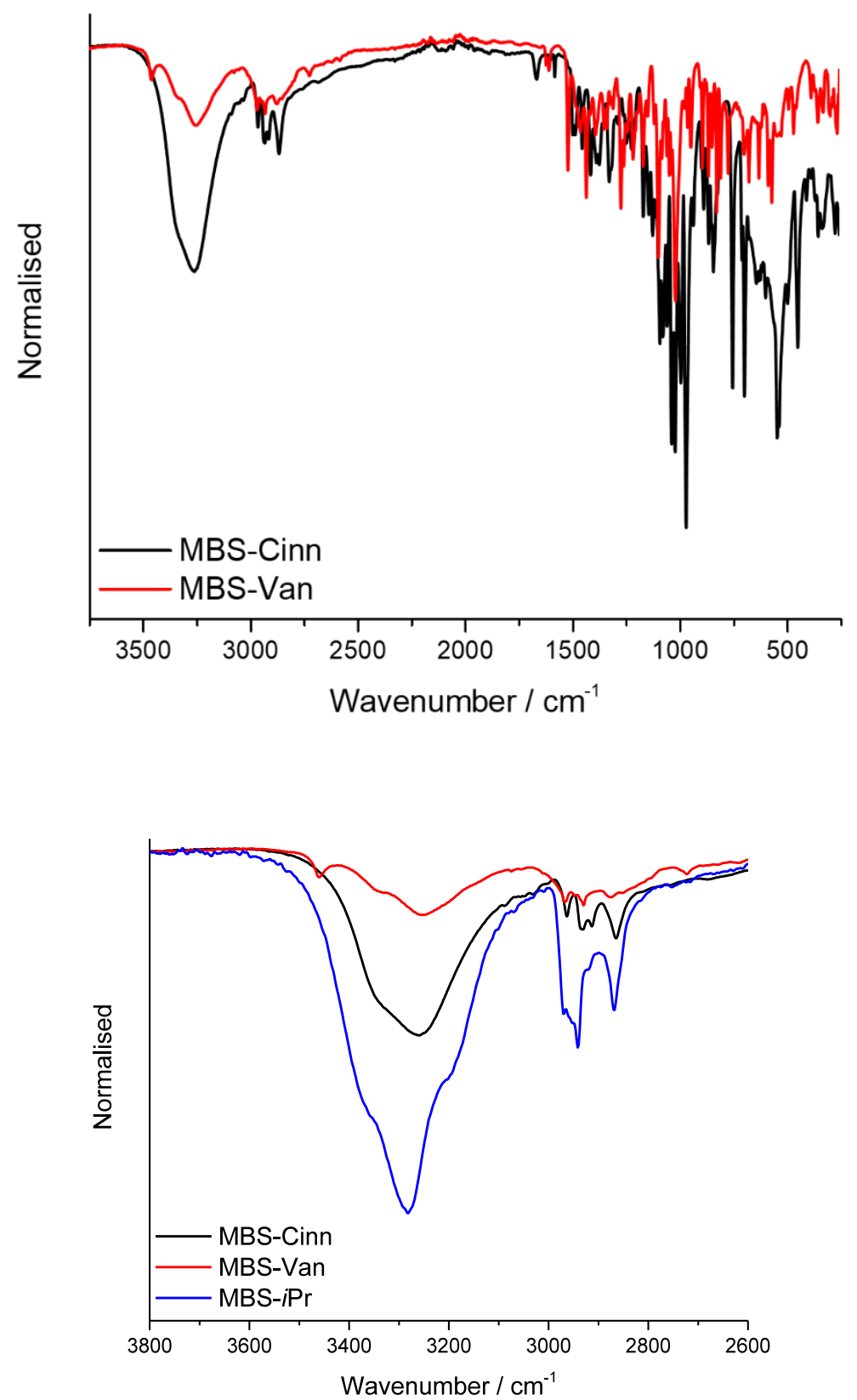

SI Fig. 36 | FTIR/ATR spectra of MBS-Cinn and MBS-Van Crystals (top) and an expansion showing the hydrogen bonding region compared with microcrystals of MBS-iPr (bottom). 
SI Table 1 | Selected hydrogen bonding parameters

\begin{tabular}{|c|c|c|c|c|}
\hline$D-H \cdots A$ & $D-\mathrm{H}(\AA ̊)$ & $H \cdots A(\AA ̊)$ & $D \cdots A(\AA ̊)$ & $D-\mathrm{H} \cdots A\left({ }^{\circ}\right)$ \\
\hline \multicolumn{5}{|l|}{ MBS-Cinn } \\
\hline $\mathrm{O} 2 \mathrm{~A}-\mathrm{H} 2 \mathrm{~A} \cdots \mathrm{O} 4 \mathrm{~B}^{\mathrm{i}}$ & 0.841 & 1.946 & $2.704(11)$ & 149.9 \\
\hline 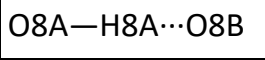 & 0.840 & 1.968 & $2.755(10)$ & 155.7 \\
\hline $\mathrm{O} 8 \mathrm{~B}-\mathrm{H} 8 \mathrm{~B} \cdots \mathrm{O} 4 \mathrm{~B}^{\mathrm{ii}}$ & 0.840 & 1.937 & $2.653(10)$ & 142.5 \\
\hline \multicolumn{5}{|l|}{ MBS-Van } \\
\hline $\mathrm{O} 2-\mathrm{H} 2 \cdots \mathrm{O} 12^{\mathrm{i}}$ & 0.840 & 1.962 & $2.7898(18)$ & 168.3 \\
\hline $\mathrm{O} 4-\mathrm{H} 4 \cdots \mathrm{O} 8^{\mathrm{iii}}$ & $0.825(19)$ & $1.84(19)$ & 2.6531 (17) & $165.1(3)$ \\
\hline $\mathrm{O} 8-\mathrm{H} 8 \cdots \mathrm{O}^{\mathrm{iv}}$ & 0.844 (19) & $1.87(2)$ & 2.6649 (17) & $155.9(2)$ \\
\hline $\mathrm{O} 12-\mathrm{H} 12 \cdots \mathrm{O}^{v}$ & $0.83(2)$ & $1.93(2)$ & 2.7580 (19) & $174.9(3)$ \\
\hline
\end{tabular}

Symmetry code(s): (i) $x-1, y+1, z$; (ii) $x-1, y, z$; (iii) $-x, y+1 / 2,-z+1$; (iv) $x, y-1, z$; (v) $x+1, y, z$. 
SI Table 2 | Single crystal X-ray Experimental details

\begin{tabular}{|c|c|c|}
\hline & MBS-Cinn & MBS-Van \\
\hline Chemical formula & $0.5\left(\mathrm{C}_{15} \mathrm{H}_{20} \mathrm{O}_{6}\right) \cdot 0.5\left(\mathrm{C}_{15} \mathrm{H}_{18} \mathrm{O}_{6}\right)$ & $\mathrm{C}_{14} \mathrm{H}_{20} \mathrm{O}_{8}$ \\
\hline$M_{\mathrm{r}}$ & 296.31 & 316.30 \\
\hline Temperature (K) & 120 & 120 \\
\hline Crystal system & Monoclinic & Monoclinic \\
\hline Space group & $P 2_{1}$ & $P 2_{1}$ \\
\hline$a, b, c(\AA)$ & 4.7673 (11), 9.5601 (19), 31.386 (6) & $8.92283(14), 4.60123$ (7), 17.4539 (3) \\
\hline$\alpha, \beta, \gamma\left({ }^{\circ}\right)$ & $90,92.31(2), 90$ & $90,92.9088(15), 90$ \\
\hline$V\left(\AA^{3}\right)$ & $1429.3(5)$ & $715.66(2)$ \\
\hline$z$ & 4 & 2 \\
\hline Radiation type & $\mathrm{Cu} K \alpha$ & Cu $K \alpha$ \\
\hline$\mu\left(\mathrm{mm}^{-1}\right)$ & 0.89 & 1.03 \\
\hline Crystal size $(\mathrm{mm})$ & $0.27 \times 0.03 \times 0.02$ & $0.20 \times 0.05 \times 0.03$ \\
\hline Reflections collected & 8745 & 9752 \\
\hline Independent reflections & 4045 & 2828 \\
\hline Reflections $[I>2 \sigma(I)]$ & 2892 & 2777 \\
\hline$R_{\text {int }}$ & 0.124 & 0.023 \\
\hline$\theta_{\max }\left({ }^{\circ}\right)$ & 58.9 & 73.5 \\
\hline$(\sin \theta / \lambda)_{\max }\left(\AA^{-1}\right)$ & 0.556 & 0.622 \\
\hline$R\left[F^{2}>2 \sigma\left(F^{2}\right)\right], w R\left(F^{2}\right), S$ & 0.088 & 0.025 \\
\hline $\mathrm{wR}\left(\mathrm{F}^{2}\right)$ [all data] & 0.224 & 0.067 \\
\hline Goodness-of-on on $F^{2}$ & 1.06 & 1.06 \\
\hline No. of reflections & 4045 & 2828 \\
\hline No. of parameters & 449 & 201 \\
\hline No. of restraints & 697 & 7 \\
\hline Largest diff. Peak/hole $\left(\mathrm{e}^{-3}{ }^{-3}\right)$ & $0.41,-0.28$ & $0.21,-0.18$ \\
\hline CCDC number & 1945762 & 1945763 \\
\hline
\end{tabular}


(a) MBS-Van

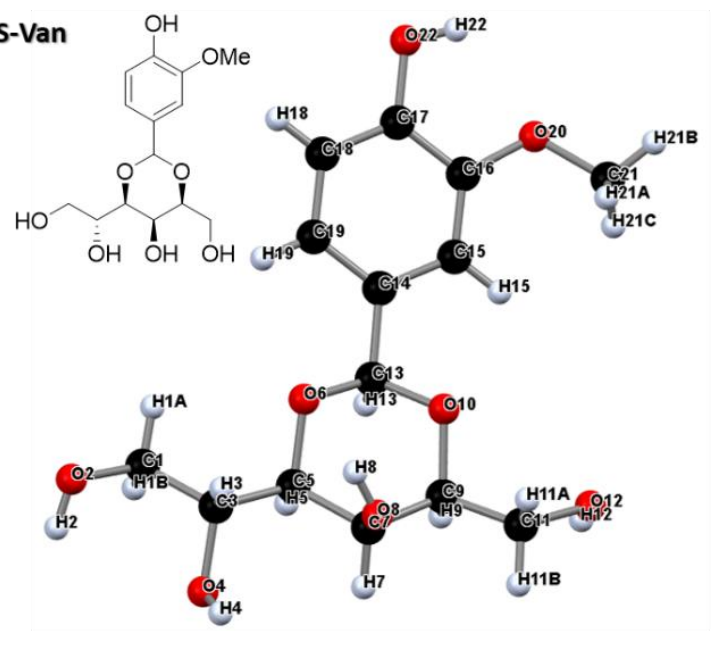

(b) MBS-Cinn

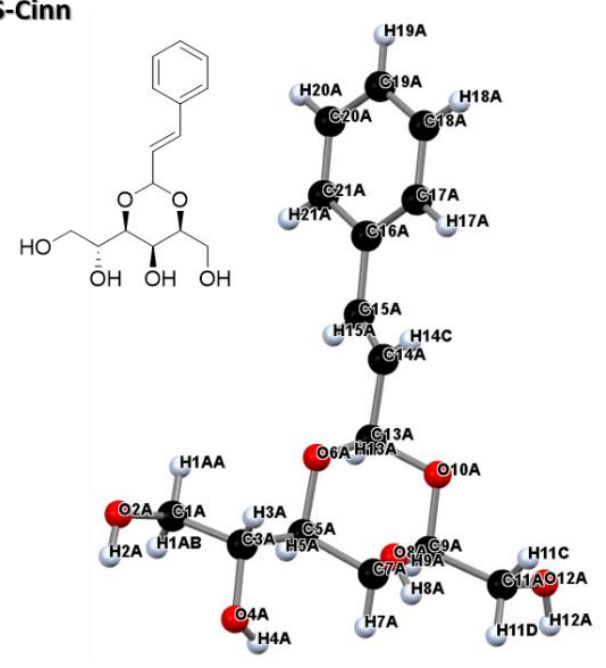

SI Fig. 37 | Crystal structures of MBS-Van and MBS-Cinn

(a) MBS-Van
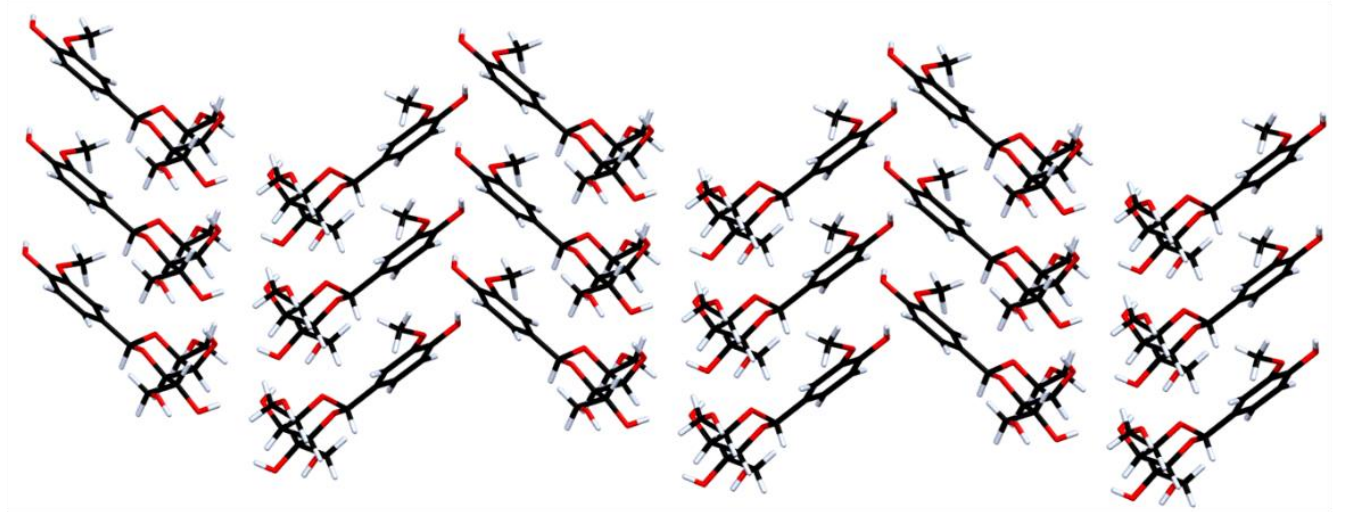

(a) MBS-Cinn

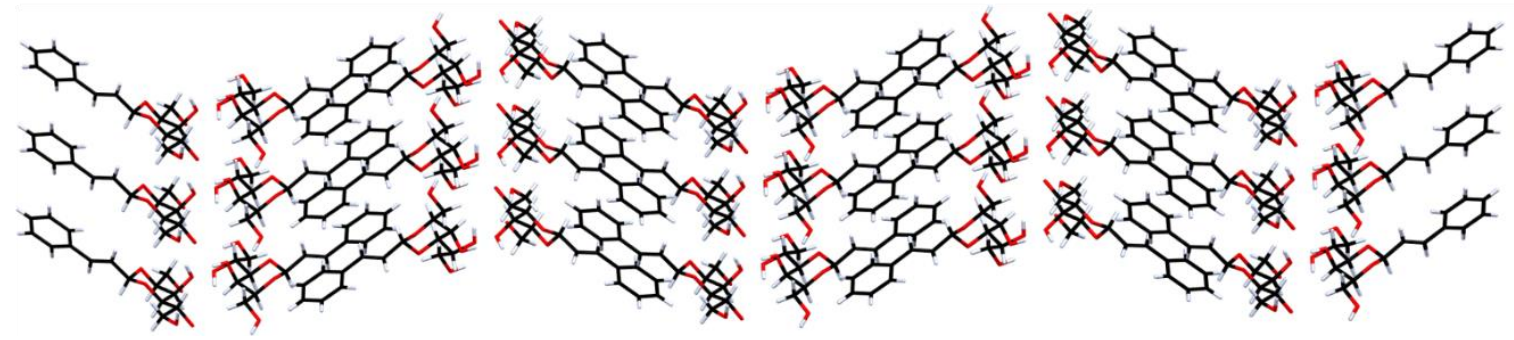

SI Fig. 38 | Packing motifs of MBS-Van and MBS-Cinn 


\section{Circular Dichroism}

(a)

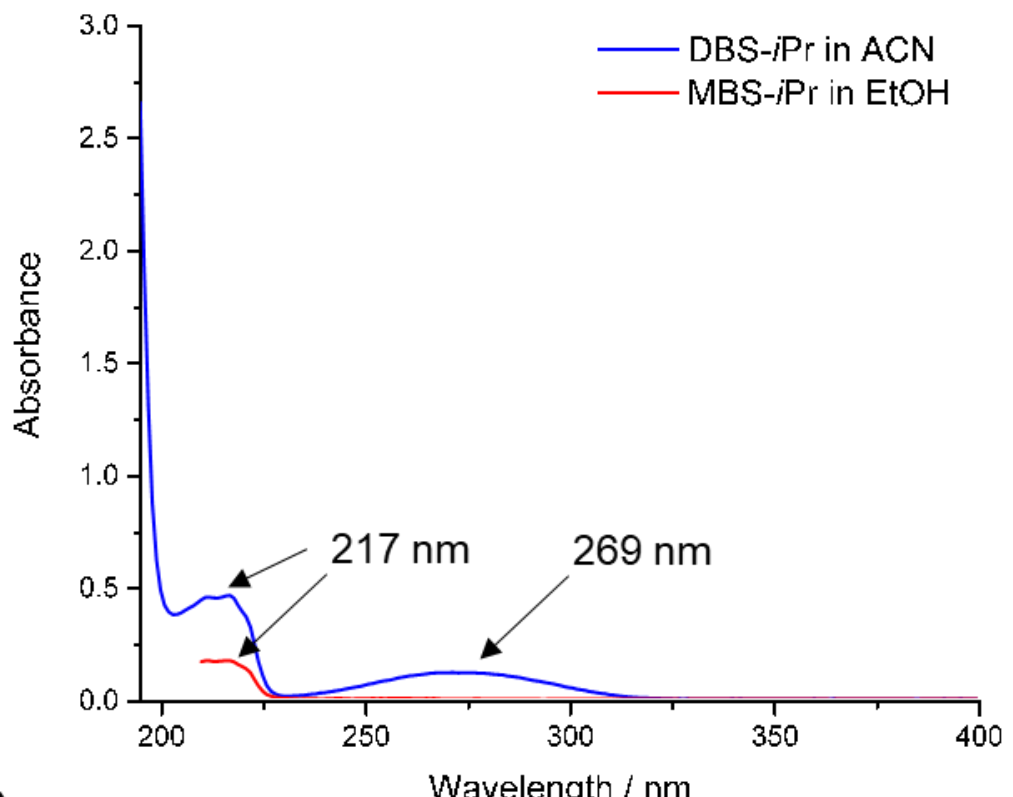

(b)

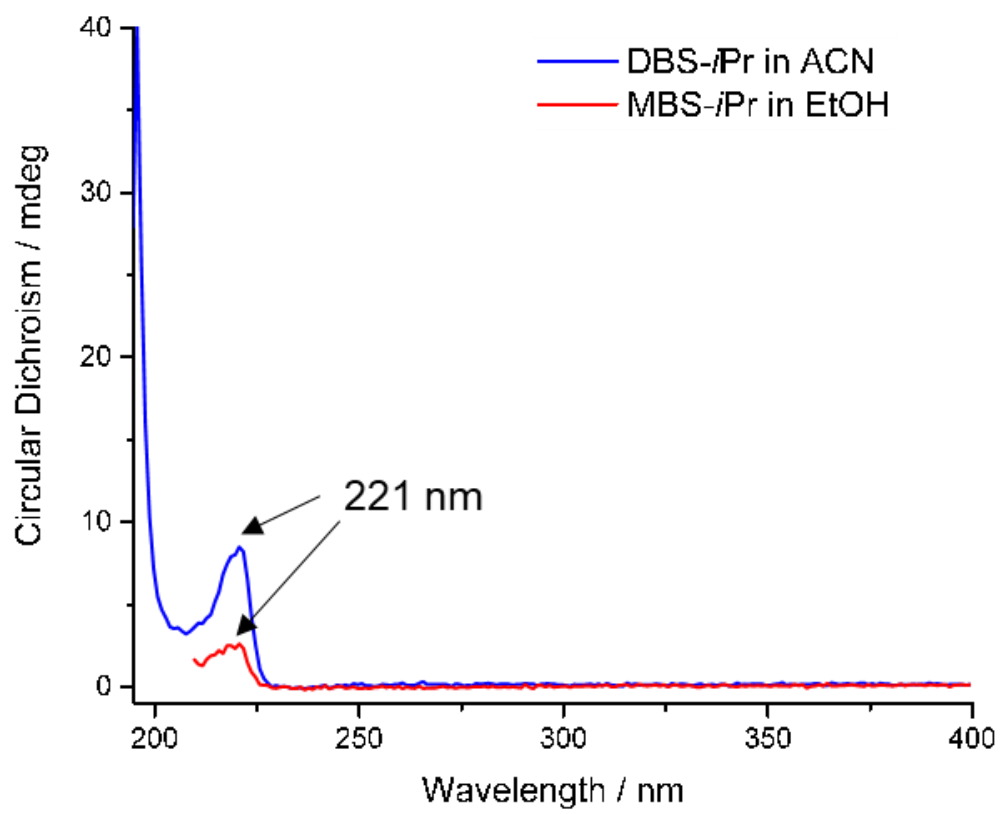

SI Fig. 39 | (a) Absorbance and (b) circular dichroism spectra for DBS-iPr in acetonitrile (blue) and MBS-iPr in ethanol (red). 


\section{References}

ESI1. J. Cosier and A. M. Glazer, J. Appl. Crystallogr., 1986, 19, 105-107.

ESI2. CrysAlisPro 1.171.40.45a (Rigaku OD, 2019).

ESI3. O. V Dolomanov, L. J. Bourhis, R. J. Gildea, J. A. K. Howard, H. Puschmann, J. Appl. Cryst. 2009, $42,339-341$.

ESI4. G. M. Sheldrick, Acta Crystallogr. A 2015, 71, 3-8.

ESI5. G. M. Sheldrick, Acta Crystallogr. C 2015, 71, 3-8.

"CheckCIF," can be found under http://checkcif.iucr.org 